WSRC-TR-2001-00004

Revision 0

\title{
Adapting The CANBERRA $\mathbf{Q}^{2}$ To Assay High-Density Solid Waste From 321-M
}

\author{
Saleem R. Salaymeh \\ and \\ Raymond A. Dewberry
}

Unclassified

Does Not Contain Unclassified Controlled Nuclear Information (UCNI)

January 8, 2001

Westinghouse Savannah River Company

Savannah River Site

Aiken, SC 29808 
WSRC-TR-2001-00004

Revision 0

Keywords:

Deactivation, NDA, Multichannel,

Transmission, Segmented, Holdup

Classification: $\mathrm{U}$

Authorized Derivative Classifier

Adapting The CANBERRA Q ${ }^{2}$ To Assay

High-Density Solid Waste From 321-M

Saleem R. Salaymeh and R. A. Dewberry

Publication Date: January 8, 2001

Saleem R. Salaymeh, Author

Date

R.A. Dewberry, Author

Date

Ann Gibbs, Technical Reviewer

Date

P. E. Filpus-Luyckx, Manager, A\&RRG-ADS

Date

Westinghouse Savannah River Company

Savannah River Site

Aiken, SC 29808 
This document was prepared in conjunction with work accomplished under Contract No.

DE-AC09-96SR18500 with the U.S. Department of Energy.

\section{DISCLAIMER}

This report was prepared as an account of work sponsored by an agency of the United States Government. Neither the United States Government nor any agency thereof, nor any of their employees, makes any warranty, express or implied, or assumes any legal liability or responsibility for the accuracy, completeness, or usefulness of any information, apparatus, product or process disclosed, or represents that its use would not infringe privately owned rights. Reference herein to any specific commercial product, process or service by trade name, trademark, manufacturer, or otherwise does not necessarily constitute or imply its endorsement, recommendation, or favoring by the United States Government or any agency thereof. The views and opinions of authors expressed herein do not necessarily state or reflect those of the United States Government or any agency thereof.

This report has been reproduced directl y from the best available copy.

Available for sale to the public, in paper, from: U.S. Department of Commerce, National Technical Information Service, 5285 Port Roy al Road, Springfield, VA 22161, phone: (800)

553-6847, fax: (703) 605-6900, email: orders@ntis.fedworld.gov online ordering: http://www.ntis.gov/ordering.htm

Available electronically at http://www.doe.gov/bridge

Available for a processing fee to U.S. Department of Energy and its contractors, in paper, from: U.S. Department of Energy, Office of Scientific and Tech nical Information, P.O. Box 62, Oak Ridge, TN 37831-0062, phone: (865 ) 576-8401, fax: (865) 576-5728, email: reports@adonis.osti.gov 


\section{ABSTRACT}

The Analytical Development Section of Savannah River Technology Center (SRTC) was requested by the Facilities Disposition Division (FDD) to determine the holdup of enriched uranium in the 321-M facility as part of an overall deactivation project of the facility. The 321-M facility was used to fabricate highly enriched uranium (HEU) fuel assemblies, lithium-aluminum target tubes, neptunium assemblies, and miscellaneous components for the production reactors. The facility also includes the 324-M storage building and the passageway connecting it to 321-M. The results of the holdup assays are essential for determining compliance with the Waste Acceptance Criteria, Material Control \& Accountability, and to meet criticality safety controls. As part of the preparation for the start of the dismantling and removal of the HEU, loose and small items were placed in 55 gal drums for shipment to Solid Waste. A large number of drums contained low to medium density materials, and 72 drums contained high-density items. The materials in the 72 drums with high-density waste included scrap metal, metal hand-tools, power tools, motors, pumps, tool boxes, computer parts, gages, etc. All the drums containing low to medium density materials were assayed using the 315-M Canberra $\mathrm{Q}^{2}$ Waste Assay System. This system is optimized for the assay of small, evenly distributed quantities of gamma emitting radionuclides. The $\mathrm{Q}^{2}$ gives the most accurate results when measuring lightly packaged, hydrocarbon-type materials such as paper, plastics, and cardboard. We developed a novel adaptation of the M-Area $Q^{2}$ using its three detectors and a U-235 transmission source to make multiple measurements of uranium-235 in each drum. The conventional $\mathrm{Q}^{2}$ combines the three detector outputs and makes a single density correction measurement based solely on sample mass. This makes the instrument suitable to assay only uniformly distributed low-density waste. We were able to adapt the instrument for assaying non-uniform high-density waste. Each detector's output was collected as a unique measurement. Using three different uranium-235 transmission source locations, we were able to identically measure U-235 gamma transmission through three drum segments. This multiple correction approach is used in segmented gamma scanners. This greatly reduces the impact that variations in the high-density materials would have on the results. This report covers measurements of uranium-235 content in high-density waste within a 55 gal container. We were able to report defensible U-235 contents in 65 of the 72 drums. Our results indicated that the 65 drums contain a total amount of $24.88 \mathrm{~g}$ of enriched uranium with values ranging from $2 \mathrm{mg}$ to $3.66 \mathrm{~g}$. This report will discuss the methodology, measurements, assumptions, and results of the U-235 content calculations for each of the 72 drums. 
WSRC-TR-2001-00004

Revision 0

January 8, 2001

Page 5 of 69

This page intentionally left blank. 


\section{TABLE OF CONTENTS}

1. INTRODUCTION................................................................................................................ 7

2. EXPERIMENTAL …................................................................................................................... 8

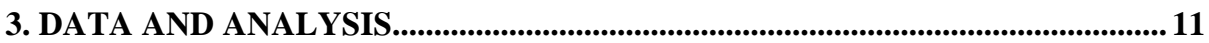

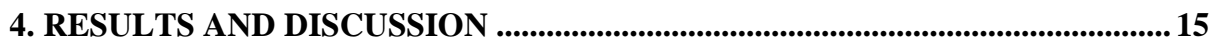

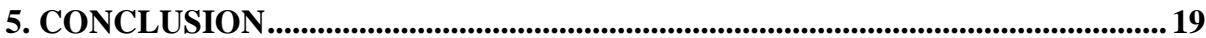

6. ACKNOWLEDGEMENTS...................................................................................................20

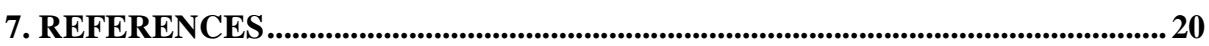

8. APPENDIX ……........................................................................................................... 21

\section{LIST OF FIGURES}

Figure 1. A photograph of the $Q^{2}$ showing its detector arrangement.............................. 8

Figure 2. A photograph of the $Q^{2}$ showing its inside and transmission source............ 10

\section{LIST OF TABLES}

Table 1. Sample Calculations for Drum FD000420..................................................... 12

Table 2. A list of all the drums and measured contents.................................................. 17 


\title{
Adapting The CANBERRA $Q^{2}$ To Assay \\ High-Density Solid Waste From 321-M
}

\author{
By Saleem R. Salaymeh and Raymond A. Dewberry \\ Westinghouse Savannah River Company \\ Savannah River Site \\ Aiken SC 29808
}

\section{INTRODUCTION}

The 321-M facility was used to fabricate enriched uranium fuel assemblies, lithiumaluminum target tubes, neptunium assemblies, and miscellaneous components for the production reactors. The facility also includes the 324-M storage building and the passageway connecting it to $321-\mathrm{M}$. The facility operated for 25 years; during this time, thousands of uranium-aluminum-alloy $(\mathrm{U}-\mathrm{Al})$ fuel tubes were produced. After the facility ceased operations in 1995, all of the easily accessible U-Al was removed from the building and only residual amounts remained. The bulk of this residue is located in the equipment that generated and handled small U-Al particles and the exhaust systems for this equipment (e.g., Chip compactor, casting furnaces, log saw, lathes A \& B, cyclone separator, Freon ${ }^{\mathrm{TM}}$ cart, riser crusher, ....etc). ${ }^{1}$

Prior to the start of the dismantling and removal of the HEU, lose and small items were placed in 55 gal drums for shipment to Solid Waste. ${ }^{2}$ A large number of drums contained low to medium density materials, and 72 drums contained high-density items. The materials in the 72 drums with high-density waste included scrap metal, metal hand-tools, power tools, motors, pumps, tool boxes, computer parts, gages, etc.

Each low level waste package presented for disposal in the E-Area Vaults must meet the radionuclide limits specified in WSRC $1 \mathrm{~S}$ WAC, 3.17. ${ }^{3}$ WSRC $1 \mathrm{~S}$, WAC $2.02^{4}$ specifies acceptable methods for quantifying waste package activity. Among the acceptable methods are Dose-to-Curie (DTC), direct sampling, and direct assay. Examples of acceptable assay equipment include use of the Canberra $\mathrm{Q}^{2}$ system as specifically cited in WAC 2.02.

Using the assay results, together with a known radiological distribution, the generator can quantify waste package isotopic activity and compare against WSRC 1S, WAC 3.17 limits to certify compliance with the radiological criteria. ${ }^{3}$

All the drums containing low to medium density materials were assayed using the 315-M Canberra $Q^{2}$ Waste Assay System. This system is optimized for the assay of small, evenly distributed quantities of gamma-emitting radionuclides. The $\mathrm{Q}^{2}$ gives the most accurate results when measuring lightly packaged, hydrocarbon-type materials such as paper, plastics, and cardboard. 
The 72 drums containing high-density waste containing enriched uranium were scheduled for assay using the passive neutron shuffler system existing in F-Area. However, due to the work priority and backlog existing at the time of assay, the F-Area shuffler was not available for use to quantify these drums.

Using neutron-counting techniques is by far the preferred method of assaying very highdensity items. To assay for HEU requires a neutron activation technique. Either of the FB-Line or M-Area Cf-shufflers would be a good choice for these assays, but neither was available. An active-well neutron coincidence counter does not have adequate sensitivity to measure U-235 in the range of milligrams or grams per drum. ${ }^{6}$ A passive neutron drum counter is not useful for measurement of U-235 content. We were constrained to use some kind of a $\gamma$-ray acquisition system to perform these requested assays. A far-field $\gamma$-ray assay was unsuitable for the same reasons that the $Q^{2}$ instrument procedure was unsuitable. The extreme densities and extreme non-uniformity of the waste made the use of a single transmission correction completely unreliable. To perform the assays we adapted the 313-M Q $\mathrm{Q}^{2}$ instrument to operate approximately like a segmented gamma scanner.

This report discusses how the Canberra $\mathrm{Q}^{2}$ was adapted for measuring U-235 content in high-density waste. It also includes the methodology, non-destructive assay (NDA) measurements, assumptions, and results of the assay of 72 drums containing high-density waste.

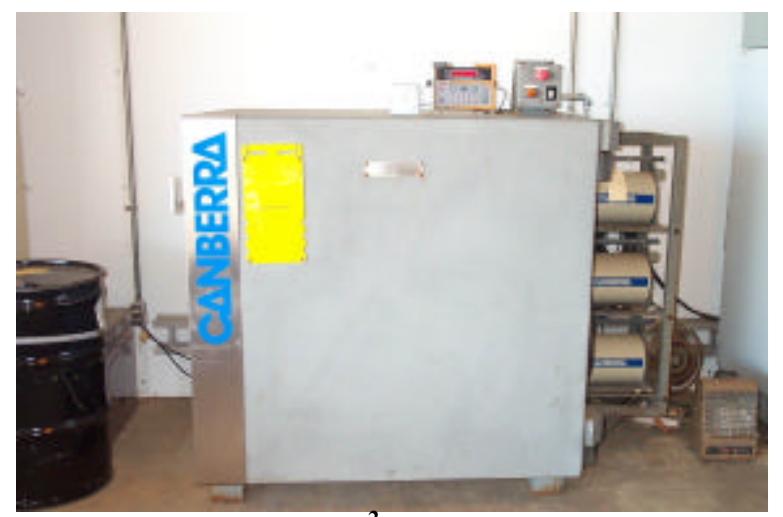

Figure 1. A photograph of the $Q^{2}$ showing its detector arrangement. 


\section{EXPERIMENTAL}

\section{Limitations of the method:}

- This system is optimized for the assay of small, evenly distributed quantities of gamma emitting radionuclides.

- The Q2 gives the most accurate results when measuring lightly packaged, hydrocarbon-type materials such as paper, plastics, and cardboard.

- The $185 \mathrm{KeV}$ will not be detected through $3 / 4$ in of steel, thus any object, such as pumps that have $>3 / 4$ " thick walls and contains internal contamination, will not be detected.

- An additional uncertainty is introduced because the detectors are not collimated. This allows cross talk between the three vertical segments, making the system effectively a hybrid between a close-field and segmented system. Therefor we have two distinct correction factors for calculating the content.

- Often the top half of the drum is empty which could lead to an error in the transmission.

- Since the waste in the drum is not homogeneous, and the drum is rotating, a metal object may be in the field of view of the detector only part of the time. The $\mathrm{k} \ln (1 / \mathrm{T})$ form of the transmission correction factor partly compensates for this uncertainty.

To accomplish the measurements requested we adapted the 313-M Canberra $\mathrm{Q}^{2}$ instrument to acquire data in a mode approximately equivalent to a three segment segmented gamma scanner. The 313-M Canberra $\mathrm{Q}^{2}$ Waste Assay System is a quantitative and qualitative gamma-ray pulse height analysis system capable of measuring very low amounts of U-235. It uses three $20 \%$ efficient intrinsic high purity germanium detectors with 7.5 liter cryostats. The sample is placed on a turntable mounted on the door of the shielding and then rotated at a nominal 10 RPM. An electronic load cell on the turntable weighs the sample and displays the result. A four-inch thick low-background steel shield surrounds the sample and detectors in all directions. The data acquisition system contains a PC-based Canberra AccuSpec Multichannel Analyzer system, appropriate high voltage power supply, and amplifiers. The three AccuSpec boards are controlled by Canberra's Genie-PC Gamma Waste Assay System. ${ }^{7}$

A photograph of the Canberra $\mathrm{Q}^{2}$ is shown in Figure 1. Note that the instrument has three vertically stacked detectors to acquire close field $\gamma$-ray data from three portions of a 55gallon drum. When operated by its normal procedure to assay for U-235, the system sums the three spectra acquired and fits the peak area in the summed spectrum to determine the detection rate for the $185-\mathrm{keV} \gamma$-ray from U-235 decay. The system then applies a predetermined detection efficiency and a density correction factor to relate detected events to measured content. ${ }^{8}$ An important feature is that the three detectors are not collimated. The transmission correction factor used by the $\mathrm{Q}^{2}$ is determined from a simple measure of the mass of the drum being assayed, and so the instrument assumes a completely uniform distribution of mass in the drum and that each detector is looking at the same transmission characteristics. For these 72 drums of solid waste those two assumptions are especially 
poor. In addition, since the drums have very high densities up to $1.3 \mathrm{~g} / \mathrm{cc}$, they are out of the reliable range for the $\mathrm{Q}^{2}$. Local densities ranged up to $8 \mathrm{~g} / \mathrm{cc}$, and so the transmission of the $185-\mathrm{keV} \gamma$-ray out of individual components of the waste will be as low as fractions of a percent. Therefore the only reliable measure of U-235 content using $\gamma$-ray acquisitions would be with a segmented scanner.

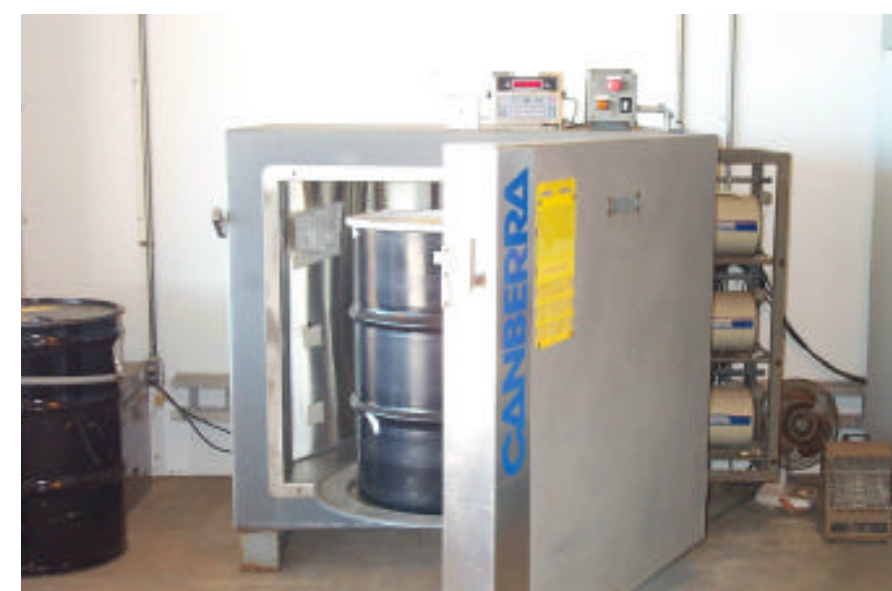

Figure 2. A photograph showing inside the $\mathbf{Q}^{2}$ and transmission source positions.

Our technique of data acquisition to assay for U-235 content in each of the 72 drums is illustrated in the photograph shown in Figure 2. For each drum a 4.85-g source of U-235 was placed inside the $\mathrm{Q}^{2}$ cavity opposite the top detector, and a $\gamma$-ray spectrum was acquired with the drum in place for $60-600$ seconds. The area under the $185-\mathrm{keV} \gamma$-ray peak and the detector livetime were obtained manually for the top detector. Then successive acquisitions were obtained with the source opposite the middle and bottom detectors, and the data for each of the middle and bottom detectors were obtained manually. Finally the drum was counted for 600 - 3600 seconds without the source present. In this way we obtained data sufficient to measure the transmission of the photons from the 4.85-g source directly through each of the three barrel segments to the detector opposite the source.

This was a very expensive technique to use to assay the 72 drums. Each drum required four counts. None of the acquisitions was automated, and all of the data were obtained manually. For each acquisition the $\mathrm{Q}^{2}$ stores the summed spectrum from all three detectors, but no single spectrum from an individual detector was ever available except on the instrument display immediately after the acquisition or when we aggressively stored it on a disk. Data were hand recorded in a laboratory notebook for subsequent analysis. We recorded data on thirteen separate days of acquisitions over a period of three months. For each distinct day of acquisition we recorded $\mathrm{T}_{0}$ spectra with the transmission source 
successively opposite each of the three detectors and with no sample drum present. These daily spectra served both as the unattenuated transmission spectra for determination of sample attenuation and as a QC check on the instrument. FDD management accepted the very large expense and the known and very large uncertainties of using this $\gamma$-ray technique in order to obtain a gram value for each of these high priority drums.

\section{Data and Analysis}

The data acquired for each drum are recorded and manipulated in the Excel $^{\mathrm{TM}}$ spreadsheet of Table 1. The first eight columns in Table 1 serve to identify the drum and to record count times and peak areas obtained and to calculate cps and uncertainties for each detector segment for the transmission data and sample only data. Columns nine through thirteen contain calculated values that we describe below in an example calculation. The spreadsheet was formatted to round all results to two places past the decimal. The data for each drum are recorded in four blocks of three where the last block of three represents the manual counts obtained in each of the three detectors from the drum-only spectra. For each drum the first three blocks of three represent the data obtained with the source present. For each day of acquisition we have recorded three blocks of three $\mathrm{T}_{0}$ data.

Looking at the first three blocks of $\mathrm{T}_{0}$ data acquired on 25 July we begin a description of the calculations. The spectrum labeled T01725 is the unattenuated spectrum going into the top detector, the spectrum labeled T02det 2 is the unattenuated spectrum going to the middle detector, and the spectrum labeled T03det 3 is the unattenuated spectrum going to the bottom detector. These three spectra had count rates of 16.99, 16.48, and $16.52 \mathrm{cps}$ respectively, as seen in column seven of Table 1. Taking the average, we record $16.66 \mathrm{cps}$ as the unattenuated count rate for each detector. 
Table 1. Sample Calculations for Drum FD000420.

\begin{tabular}{|c|c|c|c|c|c|c|c|c|c|c|c|c|}
\hline Sample & $\begin{array}{c}\text { File } \\
\text { Name }\end{array}$ & $\begin{array}{c}\text { Count } \\
\text { Time }\end{array}$ & Area & $\% \sigma$ & $\sigma$ & cps & $\sigma$ cps & $1 / T$ & $\begin{array}{c}C_{f} \\
\text { SQRT } \\
\text { of } 1 / T\end{array}$ & \begin{tabular}{|c|} 
Segment \\
Corrected \\
cps
\end{tabular} & $\begin{array}{l}C_{\text {f }} \text { of } \\
\ln 1 / T\end{array}$ & \begin{tabular}{|c|} 
Segment \\
Corrected \\
cps
\end{tabular} \\
\hline $\mathrm{T}_{01}$ & T01725 & 281.52 & 4784 & 1.49 & 71.28 & 16.99 & 0.25 & & & & & \\
\hline$T_{01}$ & T01det2 & 281.52 & 4621 & 1.52 & 70.24 & 16.41 & 0.25 & & & & & \\
\hline $\mathrm{T}_{01}$ & T01det3 & 281.52 & 3438 & 1.76 & 71.28 & 12.21 & 0.25 & & & & & \\
\hline$T_{02}$ & T02det1 & 300 & 4396 & 1.56 & \begin{tabular}{|l|}
68.58 \\
\end{tabular} & 14.65 & 0.23 & & & & & \\
\hline $\mathrm{T}_{02}$ & T02det2 & 300 & 4944 & 1.47 & 72.68 & 16.48 & 0.24 & & & & & \\
\hline $\mathrm{T}_{02}$ & T02det3 & 300 & 4385 & 1.55 & 67.97 & 14.62 & 0.23 & & & & & \\
\hline $\mathrm{T}_{03}$ & \begin{tabular}{|l|} 
T03det1 \\
\end{tabular} & 300 & 3750 & 1.72 & 64.50 & 12.50 & 0.22 & & & & & \\
\hline $\mathrm{T}_{03}$ & T03det2 & 300 & 4670 & 1.52 & 70.98 & 15.57 & 0.24 & & & & & \\
\hline $\mathrm{T}_{03}$ & T03det3 & 300 & 4955 & 1.47 & 72.84 & 16.52 & 0.24 & & & & & \\
\hline $\mathrm{T}_{\text {empty drum }}$ & Dmtdet1 & 300 & 3151 & 1.85 & 58.29 & 10.50 & 0.19 & 1.59 & 1.26 & & 1.19 & \\
\hline $\mathrm{T}_{\text {empty drum }}$ & Dmtdet2 & 300 & 3631 & 1.73 & 62.82 & 12.10 & 0.21 & 1.38 & 1.17 & & 1.13 & \\
\hline $\mathrm{T}_{\text {empty drum }}$ & Dmtdet3 & 300 & 3230 & 1.83 & 59.11 & 10.77 & 0.20 & 1.55 & 1.24 & & 1.18 & \\
\hline FD000420 & T1420D1 & 271 & 2793 & 1.98 & 55.30 & 10.31 & 0.20 & 1.71 & 1.31 & & 1.23 & \\
\hline FD000420 & T1420D2 & 271 & 493 & 5.03 & 24.80 & 1.82 & 0.09 & & & & & \\
\hline FD000420 & T1420D3 & 271 & 258 & 4.26 & 10.99 & 0.95 & 0.04 & & & & & \\
\hline FD000420 & T2420D1 & 600 & 632 & 4.45 & 28.12 & 1.05 & 0.05 & & & & & \\
\hline FD000420 & T2420D2 & 600 & 2140 & 2.36 & 50.50 & 3.57 & 0.08 & 6.41 & 2.53 & & 1.90 & \\
\hline FD000420 & T2420D3 & 600 & 1268 & 3.05 & \begin{tabular}{|l|}
38.67 \\
\end{tabular} & 2.11 & 0.06 & & & & & \\
\hline FD000420 & \begin{tabular}{|l|} 
T3420D1 \\
\end{tabular} & 600 & 1644 & 2.66 & \begin{tabular}{|l|}
43.73 \\
\end{tabular} & 2.74 & 0.07 & & & & & \\
\hline FD000420 & T3420D2 & 600 & 1381 & 2.97 & 41.02 & 2.30 & 0.07 & & & & & \\
\hline FD000420 & T3420D3 & 600 & 3325 & 1.28 & 42.56 & 5.54 & 0.07 & 3.37 & 1.83 & & 1.55 & \\
\hline FD000420 & 420D1 & 1292.3 & 719 & 4.19 & 30.13 & 0.56 & 0.02 & & & 0.73 & & 0.68 \\
\hline FD000420 & 420D2 & 1292.3 & 1252 & 3.14 & 39.31 & 0.97 & 0.03 & & & 2.45 & & 1.84 \\
\hline FD000420 & 420D3 & 1292.3 & 766 & 4.04 & 30.95 & 0.59 & 0.02 & & & 1.09 & & 0.92 \\
\hline & & & & & & & & \multicolumn{2}{|c|}{ Sum (cps) } & 4.27 & & 3.44 \\
\hline
\end{tabular}


To obtain the transmission for detector 1 for drum FD000420, we use the transmission spectrum T1420D1 and the sample only spectrum 420D1. The transmission spectrum detection rate is $10.31 \pm 0.20 \mathrm{cps}$, and the drum only detection rate is $0.56 \pm 0.02 \mathrm{cps}$. The transmission value of column nine is calculated by

$$
\mathrm{T}=(10.31-0.56) / 16.66=0.585
$$

and the transmission factor of column nine is

$$
1 / \mathrm{T}=1.71 \text {. }
$$

We perform similar calculations to obtain transmission factors of 6.41 and 3.37 for the middle and bottom detectors. We then perform two distinct calculations of U-235 content based on two distinct transmission correction form factors. The first calculation assumes that the correction factor is equal to the square root of the transmission factor. This is based on the reasoning that the transmission source photons had to pass through the entire sample, but on average the sample photons have to transit only half of the sample. In column ten of Table 1 we determine and tabulate the correction factors for each segment to be equal to the square root of $1 / \mathrm{T}$ for that segment. Therefore for sample FD420 we obtain the correction factors 1.307 for the top detector, 2.533 for the middle, and 1.835 for the bottom detector. These correction factors are then applied to the measured detection rates tabulated in column seven to obtain the corrected count rates tabulated in column eleven.

$$
\text { Corrected rate(det1,FD420) }=1.307(0.56)=0.727 \mathrm{cps} \text {. }
$$

The corrected rate for detector 2 is $2.454 \mathrm{cps}$, and the corrected rate for detector 3 is 1.088 cps. The three corrected rates are summed as shown in Table 1 . The total activity per drum is then determined by dividing by the overall $\mathrm{Q}^{2}$ detection efficiency for the $185 \mathrm{keV}$ $\gamma$-ray and by dividing by the branching ratio of 0.53 to obtain the total $U$ - 235 activity per drum. The total is listed at the bottom of Table 1 in units of decays per second. The summed efficiency for the $185-\mathrm{keV} \gamma$-ray is 0.000885 .

$$
\begin{aligned}
{[\mathrm{U}-235]_{\mathrm{FD} 420}=4.269 /(0.000885)(0.53) } & =4.269 / 0.000469 \\
& =9102 \mathrm{dps}
\end{aligned}
$$

Finally, we convert from decays/sec-drum to nCuries/drum by multiplying by 60/2220. The square root correction factor is applicable to a far field measurement where the rotated drum (sample) is much farther from the detector than the radius of the drum and where the correction factors are not far from unity. The far field correction factor considers that at large distances the photons passing all the way through from the back of the drum to the detector travel almost the same distance as those that pass from near the front of the drum to the detector. 
The next set of calculations of U-235 content is based on the transmission correction factor for a close field measurement. This correction factor recognizes that in a close field measurement by far most of the photons that reach the detector came from portions of the sample that are near the detector when they leave the sample. This correction factor considers that when the sample self-absorption is large, those photons that originate from the back side of the sample have very little chance to reach the detector. Therefore by far most of the photons that reach the detector had the short path to the detector and had to transit only a fraction of the sample. Thus the effective correction factor is much smaller than the square root of $1 / \mathrm{T}$.

From reference 9 , the correction factor is calculated by

$$
\mathrm{Cf}_{\text {(close field })} \quad=\quad-\mathrm{k} \ln (1 / \mathrm{T}) /\left[1-(1 / \mathrm{T})^{\mathrm{k}}\right],
$$

where $\mathrm{k}=\pi / 4=0.785$. As above, we perform a separate calculation of the correction factor from the measured transmission for each segment for each drum. In column twelve of Table 1, we calculate the close-field transmission correction factors. Using drum FD420 again, we perform an example calculation. For the top segment of drum FD420 we measured a transmission factor of 1.709. The calculated correction factor for this segment then becomes

$\mathrm{Cf}_{\text {(close field }) \quad=-0.785 \ln (1 / 1.709) /\left[1-(1 / 1.709)^{0.785}\right]}=-0.785(-0.535) /[1-0.656]$
$=1.225$

The correction factors for segments two and three are then 1.901 and 1.551 as shown in the appropriate elements of column twelve of Table 1. The corrected count rates are shown in column thirteen, where the corrected rate for the top segment of drum FD420, is

$$
1.225 * 0.56=0.682 \mathrm{cps} .
$$

The corrected segment rates are summed in column thirteen of Table 1, and for FD420 that sum is 3.443 cps. Dividing by the $\mathrm{Q}^{2}$ efficiency we obtain

$$
[\mathrm{U}-235]_{\mathrm{FD} 420}=3.443 / 0.000469=7340 \mathrm{dps},
$$

or 198 nCurie for drum FD420. The term 0.000469 is defined in equation 3 above. 
Each of the 72 drums was assayed in this manner. The measured data and calculated parameters are all tabulated in the Appendix, which is a reproduction of an Excel spreadsheet that performs the two transmission-corrected calculations for each drum. The measured transmission values for each segment of each drum are listed in column eight of the Appendix. The reader can note that generally the transmission values for the top segment of each drum were in the range of $1.3-2.0$, while those values for the middle and bottom portion varied widely in the range of $1.5-700$. (Some even went negative, which we discuss below.) These widely varying transmission values are a manifestation that the drums clearly were not filled with a uniform distribution of solid waste.

For most drums the top portion was approximately empty of material, and the measured transmission correction was approximately equal to that of the drum only, as shown by the empty drum correction factors listed near the middle of Table 1. The middle and bottom portions of the drums all contained material of widely varying density. From these data it is clear that individual transmission correction factors for each segment are very important for a $\gamma$-PHA assay. All of the 72 drums are out of the boundary limit of the $\mathrm{Q}^{2}$ assumption of uniform density.

\section{Results and Discussion}

We obtain two unique measures of U-235 content for each drum. One is based on a far field transmission correction factor, and one is based on a close field segmented gamma scanner correction factor, where the latter measure is without fail lower than the former. The adapted technique we have used with the $\mathrm{Q}^{2}$ is clearly not representative of a far-field measurement. It is also not exactly representative of a close field segmented scanner because the detectors are not collimated. Thus neither calculation of the correction factor is quite correct. In all cases for our reported value we have used the average with a one sigma standard deviation precision. The two measured values along with the reported value and $\mathrm{Q}^{2}$ value for each drum are listed in Table 2.

The reported values listed in the sixth column of Table 2 are equal to the average of the two calculations with an uncertainty equal to the standard deviation. In almost all cases the uncertainty of either single calculation is dominated by the uncertainty of the measured transmission factor. This is especially true when the transmission factor is large. That is, when a given transmission factor is large, its uncertainty is also large. The overall uncertainty in either single method of calculation of U-235 content can be determined by propagating the uncertainty of the transmission factors through the calculation. However we have observed that even when performing that rigorous propagation of uncertainty, the final uncertainty for each reported value is always dominated by the disagreement in the two methods of calculation. Thus there is no benefit to report the propagation of uncertainty. 
The $\mathrm{Q}^{2}$ value tabulated in column five of Table 2 is simply the instrumental value that the $\mathrm{Q}^{2}$ made from the drum-only acquisition using its own efficiency curve and correction factor based on each individual drum mass. For most of the 72 cases all three of the measured values are in fairly good agreement, indicating that while our adapted technique has significantly improved the defensibility of the measurement, it has not significantly improved the accuracy. However for several cases our measured values differ significantly from the $\mathrm{Q}^{2}$ values, and we believe ours are far superior. In seven cases we obtained physically unreal (i.e. negative) values of the transmission factor. From the form of equation (1) it is clear that this happens only when the drum-only detection rates are greater than the drum plus transmission source detection rates - or equivalently, when the transmission factors are so low that photons from the transmission source have no chance to reach the detector.

In drums where any one of the segments yielded a physically unreal transmission value, we have tabulated our measured value as "N/A". These seven drums represent cases where a $\gamma$-ray assay is not applicable. Even though in five of these seven cases the $Q^{2}$ instrument delivered a finite measured value using its own transmission corrected value based on mass, these measured values have to be considered completely unreliable and would under-report the actual contents. For those seven cases our transmission measurements have demonstrated that the sample must be considered infinitely thick with respect to the $185-\mathrm{keV} \gamma$-ray from U-235, and so any measured value based on $\gamma$-PHA is out of the boundary limits of the system. These seven drums will have to be assayed by a neutron counting system or repackaged for $\mathrm{Q}^{2}$ assay or analyzed with special attention similar to the holdup assays performed for the riser crusher and casting furnaces. ${ }^{10}$ 
Table 2. A list of all the drums and measured contents.

\begin{tabular}{|c|c|c|c|c|c|c|c|}
\hline Drum ID & Description & $\begin{array}{c}\mathbf{C}_{\mathrm{f}} \\
(\mathbf{S q R t})\end{array}$ & $\begin{array}{c}\mathbf{C}_{\mathrm{f}} \\
(\mathbf{l n} / \mathbf{T})\end{array}$ & $\overline{Q^{2}}$ & $\begin{array}{l}\text { nCi per } \\
\text { Drum }\end{array}$ & $\underset{\substack{\sigma \text { nCi per } \\
\text { Drum }}}{\sigma}$ & g/Drum \\
\hline Empty Drum & Empty Drum & 4 & 3 & & 4 & 1 & 0.00 \\
\hline FD000402 & Computer Equipment & 227 & 169 & $152 \pm 5$ & 198 & 41 & 0.09 \\
\hline FD000403 & Scrap Metal; Tools; Aluminum Cans & 421 & 332 & $255 \pm 7$ & 376 & 63 & 0.17 \\
\hline FD000405 & Computer Equipment & 12 & 9 & & 10 & 2 & 0.00 \\
\hline FD000406 & Widen Pumps (2) & 151 & 102 & $114 \pm 6$ & 126 & 35 & 0.06 \\
\hline FD000407 & Air Sample; Drop Light & 131 & 110 & $158 \pm 6$ & 120 & 15 & 0.06 \\
\hline FD000410 & $\begin{array}{l}\text { Tools; Grinding Brushes; Dolly Face } \\
\text { Cover; Computer Screen }\end{array}$ & 768 & 306 & $216 \pm 4$ & 537 & 327 & 0.25 \\
\hline FD000412 & Computer Equipment & 7 & 6 & $7 \pm 2$ & 6 & 1 & 0.00 \\
\hline FD000413 & Scrap Metal; Tools; Plastic & 132 & 99 & $181 \pm 4$ & 116 & 23 & 0.05 \\
\hline FD000414 & Computer Equipment & 4683 & 2543 & $2390 \pm 35$ & 3613 & 1513 & 1.67 \\
\hline FD000416 & Plastic; Monitor Meter & 220 & 153 & $165 \pm 4$ & 186 & 47 & 0.09 \\
\hline FD000417 & Computer Parts & 17 & 14 & $>10$ & 16 & 2 & 0.01 \\
\hline FD000418 & Scrap Metal; Tools & 327 & 253 & $352 \pm 11$ & 290 & 52 & 0.13 \\
\hline FD000420 & $\begin{array}{l}\text { Scrap Metal; Tools; Calibration Item; } \\
\text { Electric Motor; Scale; Electric Cords }\end{array}$ & 246 & 198 & $228 \pm 6$ & 222 & 34 & 0.10 \\
\hline FD000421 & $\begin{array}{l}\text { Scrap Metal; Power Supply; Meter } \\
\text { Gages }\end{array}$ & 1095 & 790 & $998+21$ & 942 & 216 & 0.44 \\
\hline FD000554 & Tool Box; Scale & 128 & 109 & $128 \pm 5$ & 118 & 13 & 0.05 \\
\hline FD000555 & $\begin{array}{l}\text { Scale; Computer Monitors; Power } \\
\text { Conditioner; Scrap Metal }\end{array}$ & 11 & 7 & $8 \pm 2$ & 9 & 3 & 0.00 \\
\hline FD000556 & Scrap Metal; Tools & 154 & 135 & 145 & 144 & 13 & 0.07 \\
\hline FD000557 & Tools; Scrap Metal & 283 & 246 & $273 \pm 9$ & 264 & 26 & 0.12 \\
\hline FD000559 & Scrap Metal; Motor & 75 & 41 & $28 \pm 3$ & 58 & 24 & 0.03 \\
\hline FD000560 & Computer Parts; Scrap Metal & 2 & 2 & $<4$ & 2 & 0 & 0.00 \\
\hline FD000561 & $\begin{array}{l}\text { Duct Hoses; Plastic Trash Cans; Fuse } \\
\text { Boxes }\end{array}$ & 991 & 524 & $524 \pm 24$ & 758 & 330 & 0.35 \\
\hline FD000562 & $\begin{array}{l}\text { Graphite Molds; Silver Aprons \& } \\
\text { Jackets; Press Plate }\end{array}$ & 2059 & 1139 & $1130 \pm 23$ & 1599 & 651 & 0.74 \\
\hline FD000563 & Scrap Metal; Tools & N/A & & & & & 0.00 \\
\hline FD000564 & $\begin{array}{l}\text { Scale; Air Gun; Power Tools; Scrap } \\
\text { Metal }\end{array}$ & 373 & 270 & $237 \pm 7$ & 322 & 73 & 0.15 \\
\hline FD000565 & Plastic Rack; Scrap Metal & 2133 & 1351 & & 1742 & 553 & 0.81 \\
\hline FD000566 & Wooden Box; Caution Cones & 684 & 590 & $620+13$ & 637 & 66 & 0.29 \\
\hline FD000567 & $\begin{array}{l}\text { Apron Jackets; Computer Parts; Air } \\
\text { Pump; Motor; Calculator }\end{array}$ & 77 & 42 & $40 \pm 5$ & 60 & 25 & 0.03 \\
\hline FD000569 & Plastic Hose; Scrap Metal & 774 & 645 & $769+16$ & 710 & 91 & 0.33 \\
\hline FD000570 & Calibrated Standards; Scrap Metal & 850 & 673 & 790 & 762 & 125 & 0.35 \\
\hline FD000571 & Scale; Scrap Metal & 8005 & 3748 & $2900 \pm 57$ & 5876 & 3010 & 2.72 \\
\hline FD000573 & Scrap Metal & 150 & 126 & $114 \pm 6$ & 138 & 17 & 0.06 \\
\hline FD000578 & Computer Parts; Scrap Metal & 4 & 3 & $<10$ & 4 & 1 & 0.00 \\
\hline FD000579 & Tool Box; Scrap Metal & 72 & 60 & $52+4$ & 66 & 8 & 0.03 \\
\hline
\end{tabular}


WSRC-TR-2001-00004

Revision 0

January 8, 2001

Page 18 of 69

\begin{tabular}{|c|c|c|c|c|c|c|c|}
\hline Drum ID & Description & $\begin{array}{c}\mathbf{C}_{\mathrm{f}} \\
(\mathbf{S q R t})\end{array}$ & $\begin{array}{c}\mathbf{C}_{\mathbf{f}} \\
(\ln / \mathbf{T})\end{array}$ & $\mathbf{Q}^{2}$ & $\begin{array}{c}\text { nCi per } \\
\text { Drum }\end{array}$ & $\begin{array}{c}\sigma \text { nCi per } \\
\text { Drum }\end{array}$ & g/Drum \\
\hline FD000580 & $\begin{array}{l}\text { Floppy Disk, Scrap Metal, Computer } \\
\text { Equipment }\end{array}$ & 23 & 18 & $15 \pm 1$ & 20 & 4 & 0.01 \\
\hline FD000581 & $\begin{array}{l}\text { Scale; Computer Equipment; Scrap } \\
\text { Metal }\end{array}$ & 48 & 33 & $31 \pm 2$ & 40 & 11 & 0.02 \\
\hline FD000582 & Scrap Aluminum; Scrap Metal & 5035 & 3021 & $2380+59$ & 4028 & 1424 & 1.86 \\
\hline FD000583 & Scrap Aluminum ; Scrap Metal & 70 & 41 & $34 \pm 4$ & 56 & 21 & 0.03 \\
\hline FD000594 & $\begin{array}{l}\text { Scrap Aluminum; Fiberglass; } \\
\text { Electrical Cords; Scrap Metal }\end{array}$ & 60 & 37 & $29 \pm 1$ & 48 & 16 & 0.02 \\
\hline FD00000595 & Container returned unused & 6 & 5 & $<10$ & 6 & 1 & 0.00 \\
\hline FD000596 & $\begin{array}{l}\text { Electrical Cords; Fiberglass; Scrap } \\
\text { Metal }\end{array}$ & 271 & 214 & $194 \pm 3$ & 242 & 40 & 0.11 \\
\hline FD000597 & Scrap Metal & 143 & 121 & $140+4$ & 132 & 16 & 0.06 \\
\hline FD000378 & No Description available & 8375 & 5702 & $9740 \pm 110$ & 7038 & 1890 & 3.26 \\
\hline FD000394 & No Description available & 8066 & 5011 & $4600 \pm 87$ & 6538 & 2160 & 3.03 \\
\hline FD000382 & No Description available & 1058 & 165 & $107 \pm 2$ & 612 & 631 & 0.28 \\
\hline FD000385 & No Description available & N/A & & $265 \pm 5$ & & & 0.00 \\
\hline FD000379 & No Description available & N/A & & & & & 0.00 \\
\hline FD000396 & No Description available & 208 & 111 & & 160 & 69 & 0.07 \\
\hline FD000399 & No Description available & 398 & 283 & & 340 & 81 & 0.16 \\
\hline FD000408 & No Description available & 103 & 80 & $77 \pm 3$ & 92 & 16 & 0.04 \\
\hline FD000397 & No Description available & 228 & 128 & $125 \pm 3$ & 178 & 71 & 0.08 \\
\hline FD000404 & No Description available & 161 & 126 & $109 \pm 6$ & 144 & 25 & 0.07 \\
\hline FD000384 & No Description available & N/A & & $595 \pm 15$ & & & 0.00 \\
\hline FD000400 & No Description available & 243 & 165 & $143 \pm 6$ & 204 & 55 & 0.09 \\
\hline FD000398 & No Description available & 904 & 417 & $351 \pm 12$ & 660 & 344 & 0.31 \\
\hline FD000415 & No Description available & 174 & 116 & $111 \pm 3$ & 145 & 41 & 0.07 \\
\hline FD000377 & No Description available & 105 & 83 & $78 \pm 5$ & 94 & 16 & 0.04 \\
\hline FD000411 & No Description available & 66 & 53 & $55 \pm 3$ & 60 & 9 & 0.03 \\
\hline FD000389 & No Description available & 5212 & 1445 & $1580 \pm 54$ & 3328 & 2664 & 1.54 \\
\hline FD000386 & No Description available & 2637 & 602 & $558 \pm 17$ & 1620 & 1439 & 0.75 \\
\hline FD000391 & No Description available & 606 & 313 & $312 \pm 11$ & 460 & 208 & 0.21 \\
\hline FD000392 & No Description available & 2271 & 1597 & $1530 \pm 26$ & 1934 & 477 & 0.90 \\
\hline FD000381 & No Description available & 220 & 96 & $82 \pm 3$ & 158 & 88 & 0.07 \\
\hline FD000380 & No Description available & N/A & & $112 \pm 4$ & & & 0.00 \\
\hline FD000401 & No Description available & 87 & 58 & $51 \pm 4$ & 72 & 21 & 0.03 \\
\hline FD000390 & No Description available & 1678 & 761 & $1200 \pm 22$ & 1220 & 648 & 0.56 \\
\hline FD000388 & No Description available & N/A & & $1880 \pm 42$ & & & 0.00 \\
\hline FD000395 & No Description available & 1464 & 844 & $768 \pm 19$ & 1154 & 438 & 0.53 \\
\hline FD000383 & No Description available & 3978 & 1866 & $2350 \pm 48$ & 2922 & 1493 & 1.35 \\
\hline FD000387 & No Description available & N/A & & $8950 \pm 110$ & & & 0.00 \\
\hline
\end{tabular}




\section{CONCLUSION}

We have used a special acquisition procedure that adapts the Canberra $Q^{2}$ instrument to a transmission corrected three-segment segmented gamma scanner to assay 72 drums of high density non-uniform solid waste from the 321-M deactivation project. We have reported defensible U-235 contents in 65 of the 72 drums. The measured values range from $4 \mathrm{nCi} / \mathrm{drum}(2 \mathrm{mg})$ to $7040 \mathrm{nCi} / \mathrm{drum}(3.66 \mathrm{~g}$ ) with one sigma uncertainties generally of about $20 \%$. Seven drums contained solid waste of such extreme density that we could obtain no finite transmission correction. That is, these seven drums contained waste that in at least one of the acquisition segments was infinitely thick to the $185 \mathrm{keV} \gamma$-ray. As discussed in the Introduction, these seven drums must be assayed by a neutron-based counting instrument such as a californium shuffler.

Our special acquisition procedure used a $4.85 \mathrm{~g}$ source of U-235 as a movable and removable transmission source so that we could obtain a transmission measurement for each of three segments in each of the 72 drums of solid waste. The measured transmission factors varied over the range of 1.3 up to almost 800 , clearly demonstrating extreme nonuniformity in the waste assayed. The widely varying transmission factors also clearly demonstrate that the measured values obtained from the normal operation of the $\mathrm{Q}^{2}$ would be completely indefensible for these drums.

In our determinations of U-235 content we have used two distinct calculations of the transmission correction factor. Because the acquisition configuration is an adapted one, neither method of calculation quite fits the physical situation. Therefore our reported values are based on the resulting average of the two calculations, and the reported uncertainty for each value is equal to the standard deviation of the average. This standard deviation dominates the uncertainty (imprecision) of each individual measurement so that it was not necessary to propagate the individual measurement uncertainties through for each calculation. 


\section{ACKNOWLEDGEMENTS}

The authors would like to thank Ann Gibbs for the many discussions and for providing technical review. The authors would like to extend their sincere thanks to Henry Burruss and Ernie All for scheduling and providing the operations support that made it possible for us to conduct this work.

\section{REFERENCES}

1. D. L. Honkonen, "Nuclear Criticality Safety Evaluation (NCSE): Enriched Uranium Removal From Building 321-M,” N-NCS-G-00051, February 2000.

2. Deactivation Project Plan 321-M Fuel Fabrication Facility, V-PMP-M-00004, January 2000.

3. WSRC 1S Manual, Procedure WAC 3.17, "Low Level Radioactive Waste Acceptance Criteria," October 2000.

4. WSRC 1S Manual, Procedure WAC 2.02, "Low Level, Hazardous, TRU, Mixed and PCB Waste Characterization Requirements," June 2000.

5. SRTC L7.13 Manual, Procedure 004, “Assay/Validation of SRTC Radioactive Waste," February 2000.

6. E. T. Sadowski, J. C. Griffin and P. M. Rinard, "Comparison of the Savannah River Site Billet Active Well Coincidence Counter and Two Californium Shufflers," Proceedings of the $32^{\text {nd }}$ Annual INMM Conference, July 1991.

7. Canberra Industries, “ $\mathrm{Q}^{2}$ Low Level Waste Assay System Operation Manual," October 1996.

8. R. Gianakos and M. Patterson, "Factory Efficiency Calibration of the Canberra $\mathrm{Q}^{2}$ System for WSRC," August 1994.

9. J. L. Parker, "Waste and Residue Non Destructive Assay Measurements," Safeguards Science and Technology Group (NIS-5), Los Alamos National Laboratory, June 1996.

10. S. R Salaymeh and R. A. Dewberry, "HEU Holdup Measurements in 321-M U-Al Casting Furnaces and Riser Crusher," WSRC-TR-2000-00431, October 2000. 
WSRC-TR-2001-00004 Revision 0

January 8, 2001 Page 21 of 69

\section{APPENDIX}

\begin{tabular}{|c|c|c|c|c|c|c|c|c|c|c|c|c|c|c|c|c|c|}
\hline Sample & File Name & \begin{tabular}{|l|} 
Count \\
Time
\end{tabular} & Area & $\% \sigma$ & $\sigma$ & cps & $\sigma \mathrm{cps}$ & $1 / T$ & \begin{tabular}{|c|} 
Cf $=$ \\
SQRT( \\
1/T)
\end{tabular} & $\begin{array}{l}\text { Corr. } \\
\text { cps }\end{array}$ & $\begin{array}{l}\text { Corr. } \\
\text { Sum } \\
\text { (cps) }\end{array}$ & $\begin{array}{c}\text { Drum } \\
\text { Activity } \\
\text { (dps) }\end{array}$ & $\begin{array}{c}\text { Drum } \\
\text { Activity } \\
\text { (nCi) }\end{array}$ & $\begin{array}{l}C_{f} \text { of } \\
\ln 1 / T\end{array}$ & $\begin{array}{l}\text { Corr. } \\
\text { Sum } \\
\text { (cps) }\end{array}$ & $\begin{array}{c}\text { Drum } \\
\text { Activity } \\
\text { (dps) }\end{array}$ & $\begin{array}{c}\text { Drum } \\
\text { Activity } \\
\text { (nCi) }\end{array}$ \\
\hline$T_{01}$ & T01725 & 281.52 & 4784 & 1.49 & 71.28 & 16.99 & 0.25 & & & & & & & & & & \\
\hline$T_{01}$ & T01det2 & 281.52 & 4621 & 1.52 & 70.24 & 16.41 & 0.25 & & & & & & & & & & \\
\hline$T_{01}$ & T01det3 & 281.52 & 3438 & 1.76 & 71.28 & 12.21 & 0.25 & & & & & & & & & & \\
\hline$T_{02}$ & T02det1 & 300 & 4396 & 156 & 68.58 & 1465 & 023 & & & & & & & & & & \\
\hline$T_{02}$ & T02det2 & 300 & 4944 & 1.47 & 72.68 & 16.48 & 0.24 & & & & & & & & & & \\
\hline$T_{02}$ & T02det3 & 300 & 4385 & 1.55 & 67.97 & 14.62 & 0.23 & & & & & & & & & & \\
\hline & & & & & & & & & & & & & & & & & \\
\hline$T_{03}$ & T03det1 & 300 & 3750 & 1.72 & 64.50 & 12.50 & 0.22 & & & & & & & & & & \\
\hline$T_{03}$ & T03det2 & 300 & 4670 & 1.52 & 70.98 & 15.57 & \begin{tabular}{|l|}
0.24 \\
\end{tabular} & & & & & & & & & & \\
\hline$T_{03}$ & T03det3 & 300 & 4955 & 1.47 & 72.84 & 16.52 & 0.24 & & & & & & & & & & \\
\hline & & & & & & & & 16.66 & & & & & & & & & \\
\hline $\mathrm{T}_{\text {empty drum }}$ & DMTdet1 & 300 & 3151 & 1.85 & 58.29 & 10.50 & 0.19 & 1.59 & 1.26 & & & & & 1.19 & & & \\
\hline $\mathrm{T}_{\text {empty drum }}$ & DMTdet2 & 300 & 3631 & 1.73 & 62.82 & 12.10 & 0.21 & 1.38 & 1.17 & & & & & 1.13 & & & \\
\hline $\mathrm{T}_{\text {empty drum }}$ & DMTdet3 & 300 & 3230 & 1.83 & 59.11 & 10.77 & 0.20 & 1.55 & 1.24 & & & & & 1.18 & & & \\
\hline FD000420 & T1420D1 & 271 & 2793 & 1.98 & 55.30 & 10.31 & 0.20 & 1.71 & \begin{tabular}{l|}
1.307 \\
\end{tabular} & & & & & 1.2251 & & & \\
\hline FD000420 & T1420D2 & 271 & 493 & 5.03 & 24.80 & 1.82 & 0.09 & & & & & & & & & & \\
\hline FD000420 & T1420D3 & 271 & 258 & 4.26 & 10.99 & 0.95 & 0.04 & & & & & & & & & & \\
\hline FD000420 & T2420D1 & 600 & 632 & 4.45 & 28.12 & 1.05 & 0.05 & & & & & & & & & & \\
\hline FD000420 & T2420D2 & 600 & 2140 & 2.36 & 50.50 & 3.57 & 0.08 & 6.41 & 2.533 & & & & & 1.9009 & & & \\
\hline FD000420 & T2420D3 & 600 & 1268 & 3.05 & 38.67 & 2.11 & 0.06 & & & & & & & & & & \\
\hline
\end{tabular}


WSRC-TR-2001-00004

Revision 0

January 8, 200

Page 22 of 69

\begin{tabular}{|c|c|c|c|c|c|c|c|c|c|c|c|c|c|c|c|c|c|}
\hline Sample & File Name & $\begin{array}{c}\text { Count } \\
\text { Time }\end{array}$ & Area & $\% \sigma$ & $\sigma$ & cps & $\sigma \mathrm{cps}$ & $1 / \mathrm{T}$ & \begin{tabular}{|c|} 
Cf $=$ \\
SQRT( \\
1/T)
\end{tabular} & $\begin{array}{c}\text { Corr. } \\
\text { cps }\end{array}$ & $\begin{array}{l}\text { Corr. } \\
\text { Sum } \\
\text { (cps) }\end{array}$ & $\begin{array}{c}\text { Drum } \\
\text { Activity } \\
\text { (dps) }\end{array}$ & $\begin{array}{c}\text { Drum } \\
\text { Activity } \\
\text { (nCi) }\end{array}$ & $\begin{array}{l}C_{f} \text { of } \\
\ln 1 / T\end{array}$ & $\begin{array}{l}\text { Corr. } \\
\text { Sum } \\
\text { (cps) }\end{array}$ & \begin{tabular}{|c|} 
Drum \\
Activity \\
(dps)
\end{tabular} & $\begin{array}{c}\text { Drum } \\
\text { Activity } \\
\text { (nCi) }\end{array}$ \\
\hline FD000420 & T3420D1 & 600 & 1644 & 2.66 & 43.73 & 2.74 & 0.07 & & & & & & & & & & \\
\hline FD000420 & T3420D2 & 600 & 1381 & 2.97 & 41.02 & 2.30 & 0.07 & & & & & & & & & & \\
\hline FD000420 & T3420D3 & 600 & 3325 & 1.28 & 42.56 & 5.54 & 0.07 & 3.37 & 1.835 & & & & & 1.5511 & & & \\
\hline FD000420 & 420D1 & 1292.3 & 719 & 4.19 & 30.13 & 0.56 & 0.02 & & & 0.73 & & & & 0.6816 & & & \\
\hline FD000420 & 420D2 & 1292.3 & 1252 & 3.14 & 39.31 & 0.97 & 0.03 & & & 2.45 & 4.269 & 9101.5 & 245.74 & 1.8416 & 3.443 & 7340.1 & 198.18 \\
\hline FD000420 & 420D3 & 1292.3 & 766 & 4.04 & 30.95 & 0.59 & 0.02 & & & 1.09 & & & & 0.9194 & & & \\
\hline & & & & 0.785 & & & & & & & & & & & & & \\
\hline & & & & & & & & & & & & & & & & & \\
\hline FD000413 & T1413D1 & 347.45 & 4429 & 1.56 & 69.09 & 12.75 & 0.20 & 1.36 & 1.168 & & & & & 1.1268 & & & \\
\hline FD000413 & T1413D2 & 347.45 & 659 & 4.27 & 28.14 & 1.90 & 0.08 & & & & & & & & & & \\
\hline FD000413 & T1413D3 & 347.45 & 135 & 10.92 & 14.74 & 0.39 & 0.04 & & & & & & & & & & \\
\hline & & & & & & & & & & & & & & & & & \\
\hline FD000413 & T2413D1 & 600 & 1611 & 2.66 & 42.85 & 2.69 & 0.07 & & & & & & & & & & \\
\hline FD000413 & T2413D2 & 600 & 929 & 3.97 & 36.88 & 1.55 & 0.06 & 13.88 & 3.726 & & & & & 2.365 & & & \\
\hline FD000413 & T2413D3 & 600 & 709 & 4.23 & 29.99 & 1.18 & 0.05 & & & & & & & & & & \\
\hline & & & & & & & & & & & & & & & & & \\
\hline FD000413 & T3413D1 & 600 & 478 & 5.16 & 24.66 & 0.80 & 0.04 & & & & & & & & & & \\
\hline FD000413 & T3413D2 & 600 & 882 & 3.82 & 33.69 & 1.47 & 0.06 & & & & & & & & & & \\
\hline FD000413 & T3413D3 & 600 & 1374 & 2.96 & 40.67 & 2.29 & 0.07 & 7.71 & 2.776 & & & & & 2.0069 & & & \\
\hline & & & & & & & & & & & & & & & & & \\
\hline FD000413 & 413D1 & 4166 & 2213 & 2.37 & 52.45 & 0.53 & 0.01 & & & 0.62 & & & & 0.5986 & & & \\
\hline FD000413 & 413D2 & 4166 & 1450 & 3.14 & 45.53 & 0.35 & \begin{tabular}{|l|}
0.01 \\
\end{tabular} & & & 1.30 & 2.271 & 4842.3 & 130.74 & 0.8231 & 1.677 & 3576.7 & 96.572 \\
\hline FD000413 & 413D3 & 4166 & 531 & 5.65 & 30.00 & 0.13 & 0.01 & & & 0.35 & & & & 0.2558 & & & \\
\hline & & & & & & & & & & & & & & & & & \\
\hline & & & & & & & & & & & & & & & & & \\
\hline FD000410 & T1410D1 & 254.67 & 3047 & 1.9 & 57.89 & 11.96 & 0.23 & 1.43 & 1.195 & & & & & 1.1461 & & & \\
\hline FD000410 & T1410D2 & 254.67 & 2047 & 2.38 & 48.72 & 8.04 & 0.19 & & & & & & & & & & \\
\hline FD000410 & T1410D3 & 254.67 & 515 & 4.46 & 22.97 & 2.02 & \begin{tabular}{|l|}
0.09 \\
\end{tabular} & & & & & & & & & & \\
\hline
\end{tabular}


WSRC-TR-2001-00004

Revision 0

January 8, 200

Page 23 of 69

\begin{tabular}{|c|c|c|c|c|c|c|c|c|c|c|c|c|c|c|c|c|c|}
\hline Sample & File Name & $\begin{array}{l}\text { Count } \\
\text { Time }\end{array}$ & Area & $\% \sigma$ & $\sigma$ & cps & $\sigma$ cps & $1 / T$ & $\begin{array}{c}\text { Cf }= \\
\text { SQRT( }( \\
\text { 1/T) }\end{array}$ & $\begin{array}{c}\text { Corr. } \\
\text { cps }\end{array}$ & $\begin{array}{l}\text { Corr. } \\
\text { Sum } \\
\text { (cps) }\end{array}$ & \begin{tabular}{|c|} 
Drum \\
Activity \\
(dps)
\end{tabular} & \begin{tabular}{|c|} 
Drum \\
Activity \\
(nCi)
\end{tabular} & $\begin{array}{l}C_{f} \text { of } \\
\ln 1 / T\end{array}$ & $\begin{array}{l}\text { Corr. } \\
\text { Sum } \\
\text { (cps) }\end{array}$ & \begin{tabular}{|c|} 
Drum \\
Activity \\
(dps)
\end{tabular} & \begin{tabular}{|c|} 
Drum \\
Activity \\
(nCi)
\end{tabular} \\
\hline FD000410 & T2410D1 & 600 & 4955 & 1.49 & 73.83 & 8.26 & 0.12 & & & & & & & & & & \\
\hline FD000410 & T2410D2 & 600 & 2533 & 2.2 & 55.73 & 4.22 & 0.09 & 4.94 & 2.222 & & & & & 1.7542 & & & \\
\hline \multirow[t]{2}{*}{ FD000410 } & T2410D3 & 600 & 1310 & 3.01 & 39.43 & 2.18 & 0.07 & & & & & & & & & & \\
\hline & & & & & & & & & & & & & & & & & \\
\hline FD000410 & T3410D1 & 600 & 1299 & 2.99 & 38.84 & 2.17 & 0.06 & & & & & & & & & & \\
\hline FD000410 & T3410D2 & 600 & 1554 & 2.84 & 44.13 & 2.59 & 0.07 & & & & & & & & & & \\
\hline \multirow[t]{2}{*}{ FD000410 } & T3410D3 & 600 & 578 & 4.72 & 27.28 & 0.96 & 0.05 & 163.46 & 12.78 & & & & & 4.0754 & & & \\
\hline & & & & & & & & & & & & & & & & & \\
\hline FD000410 & 410D1 & 3600 & 1035 & 3.78 & 39.12 & 0.29 & 0.01 & & & 0.34 & & & & 0.3295 & & & \\
\hline FD000410 & 410D2 & 3600 & 3045 & 2.1 & 63.95 & 0.85 & 0.02 & & & 1.88 & 13.24 & 28221 & 761.96 & 1.4838 & 5.324 & 11351 & 306.49 \\
\hline \multirow[t]{3}{*}{ FD000410 } & 410D3 & 3600 & 3101 & 1.98 & 61.40 & 0.86 & 0.02 & & & 11.01 & & & & 3.5105 & & & \\
\hline & & & & & & & & & & & & & & & & & \\
\hline & & & & & & & & & & & & & & & & & \\
\hline FD000594 & T1594D1 & 229.1 & 2919 & 1.93 & 56.34 & 12.74 & 0.25 & 1.31 & 1.147 & & & & & 1.1112 & & & \\
\hline FD000594 & T1594D2 & 229.1 & 1612 & 2.66 & 42.88 & 7.04 & 0.19 & & & & & & & & & & \\
\hline \multirow[t]{2}{*}{ FD000594 } & T1594D3 & 229.1 & 171 & 8.93 & 15.27 & 0.75 & 0.07 & & & & & & & & & & \\
\hline & & & & & & & & & & & & & & & & & \\
\hline FD000594 & T2594D1 & 600 & 5460 & 1.55 & 84.63 & 9.10 & 0.14 & & & & & & & & & & \\
\hline FD000594 & T2594D2 & 600 & 819 & 4.12 & 33.74 & 1.37 & 0.06 & 13.59 & 3.687 & & & & & 2.3517 & & & \\
\hline \multirow[t]{2}{*}{ FD000594 } & T2594D3 & 600 & 367 & 6.14 & 22.53 & 0.61 & 0.04 & & & & & & & & & & \\
\hline & & & & & & & & & & & & & & & & & \\
\hline FD000594 & T3594D1 & 600 & 711 & 4.21 & 29.93 & 1.19 & 0.05 & & & & & & & & & & \\
\hline FD000594 & T3594D2 & 600 & 501 & 5.95 & 29.81 & 0.84 & 0.05 & & & & & & & & & & \\
\hline \multirow[t]{2}{*}{ FD000594 } & T3594D3 & 600 & 458 & 5.31 & 24.32 & 0.76 & 0.04 & 24.80 & 4.98 & & & & & 2.7408 & & & \\
\hline & & & & & & & & & & & & & & & & & \\
\hline FD000594 & 594D1 & 6000 & 397 & 7.47 & 29.66 & 0.07 & 0.00 & & & 0.08 & & & & 0.0735 & & & \\
\hline FD000594 & 594D2 & 6000 & 835 & 4.98 & 41.58 & 0.14 & 0.01 & & & 0.51 & 1.044 & 2225.5 & 60.089 & 0.3273 & 0.651 & 1388.4 & 37.486 \\
\hline FD000594 & 594D3 & 6000 & 548 & 5.89 & 32.28 & 0.09 & 0.01 & & & 0.45 & & & & 0.2503 & & & \\
\hline & & & & & & & & & & & & & & & & & \\
\hline
\end{tabular}


WSRC-TR-2001-00004

Revision 0

January 8, 2001

Page 24 of 69

\begin{tabular}{|c|c|c|c|c|c|c|c|c|c|c|c|c|c|c|c|c|c|}
\hline Sample & File Name & \begin{tabular}{|l|} 
Count \\
Time
\end{tabular} & Area & $\% \sigma$ & $\sigma$ & cps & $\sigma$ cps & $1 / \mathbf{T}$ & $\begin{array}{c}\text { Cf }= \\
\text { SQRT( }( \\
\text { 1/T) }\end{array}$ & $\begin{array}{c}\text { Corr. } \\
\text { cps }\end{array}$ & $\begin{array}{l}\text { Corr. } \\
\text { Sum } \\
\text { (cps) }\end{array}$ & \begin{tabular}{|c|} 
Drum \\
Activity \\
(dps)
\end{tabular} & \begin{tabular}{|c|} 
Drum \\
Activity \\
(nCi)
\end{tabular} & $\begin{array}{l}C_{f} \text { of } \\
\ln 1 / T\end{array}$ & $\begin{array}{l}\text { Corr. } \\
\text { Sum } \\
\text { (cps) }\end{array}$ & $\begin{array}{c}\text { Drum } \\
\text { Activity } \\
\text { (dps) }\end{array}$ & \begin{tabular}{|c|} 
Drum \\
Activity \\
(nCi)
\end{tabular} \\
\hline \multirow[t]{3}{*}{ T01 } & T01det1 & 100 & 1577 & 2.57 & 41 & 15.77 & 0.41 & & & & & & & & & & \\
\hline & T01det2 & 100 & 1576 & 2.56 & 40 & 15.76 & 0.4 & & & & & & & & & & \\
\hline & T01det3 & 100 & 1203 & 2.96 & 36 & 12.03 & 0.36 & & & & & & & & & & \\
\hline & & & & & & & & & & & & & & & & & \\
\hline \multirow[t]{4}{*}{ T02 } & T02det1 & 100 & 1360 & 2.76 & 38 & 13.6 & 0.38 & & & & & & & & & & \\
\hline & T02det2 & 100 & 1634 & 2.52 & 41 & 16.34 & 0.41 & & & & & & & & & & \\
\hline & T02det3 & 100 & 1610 & 2.55 & 41 & 16.1 & 0.41 & & & & & & & & & & \\
\hline & & & & & & & & & & & & & & & & & \\
\hline \multirow[t]{4}{*}{ T03 } & T03det1 & 100 & 1055 & 3.18 & 34 & 10.55 & 0.34 & & & & & & & & & & \\
\hline & T03det2 & 100 & 1489 & 2.68 & 40 & 14.89 & 0.4 & & & & & & & & & & \\
\hline & T03det3 & 100 & 1714 & 2.49 & 43 & 17.14 & 0.43 & & & & & & & & & & \\
\hline & & & & & & & & 16.42 & & & & & & & & & \\
\hline \multirow[t]{17}{*}{ FD406 } & T1406D1 & 281.11 & 3557 & 1.74 & 61.89 & 12.65 & 0.22 & 1.33 & 1.153 & & & & & $\begin{array}{ll}1.1162 \\
\end{array}$ & & & \\
\hline & T1406D2 & 281.11 & 2107 & 2.31 & 48.67 & 7.495 & 0.17 & & & & & & & & & & \\
\hline & T1406D3 & 281.11 & 672 & 4.1 & 27.55 & 2.391 & $\begin{array}{ll}0.1 \\
\end{array}$ & & & & & & & & & & \\
\hline & & & & & & & & & & & & & & & & & \\
\hline & T2406D1 & 300 & 2298 & 2.19 & 50.33 & 7.66 & \begin{tabular}{|l|}
0.17 \\
\end{tabular} & & & & & & & & & & \\
\hline & T2406D2 & 300 & 2296 & 2.21 & 50.74 & 7.653 & 0.17 & 2.30 & 1.517 & & & & & 1.3624 & & & \\
\hline & T2406D3 & 300 & 132 & 10.56 & 13.94 & 0.44 & \begin{tabular}{|l|}
0.05 \\
\end{tabular} & & & & & & & & & & \\
\hline & & & & & & & & & & & & & & & & & \\
\hline & T3406D1 & 416.52 & 1528 & 2.73 & 41.71 & 3.668 & 0.1 & & & & & & & & & & \\
\hline & T3406D2 & 416.52 & 711 & 4.04 & 28.72 & 1.707 & \begin{tabular}{|l|}
0.07 \\
\end{tabular} & & & & & & & & & & \\
\hline & T3406D3 & 416.52 & 278 & 6.72 & 18.68 & 0.667 & \begin{tabular}{|l|}
0.04 \\
\end{tabular} & 38.26 & 6.185 & & & & & 3.0345 & & & \\
\hline & & & & & & & & & & & & & & & & & \\
\hline & 406D1 & 600 & 188 & 8.13 & \begin{tabular}{|l|}
15.28 \\
\end{tabular} & 0.313 & \begin{tabular}{|l|}
0.03 \\
\end{tabular} & & & 0.36 & & & & 0.3497 & & & \\
\hline & 406D2 & 600 & 310 & 6.5 & 20.15 & 0.517 & \begin{tabular}{|l|}
0.03 \\
\end{tabular} & & & 0.78 & 2.619 & 5584.6 & 150.78 & 0.7039 & 1.777 & 3788.6 & 102.29 \\
\hline & 406D3 & 600 & 143 & 10.08 & \begin{tabular}{l|}
14.41 \\
\end{tabular} & 0.238 & \begin{tabular}{|l|}
0.02 \\
\end{tabular} & & & 1.47 & & & & $\begin{array}{ll}0.7232 \\
\end{array}$ & & & \\
\hline & & & & & & & & & & & & & & & & & \\
\hline & & & & & & & & & & & & & & & & & \\
\hline
\end{tabular}


WSRC-TR-2001-00004

Revision 0

January 8, 2001

Page 25 of 69

\begin{tabular}{|c|c|c|c|c|c|c|c|c|c|c|c|c|c|c|c|c|c|}
\hline Sample & File Name & $\begin{array}{l}\text { Count } \\
\text { Time }\end{array}$ & Area & $\% \sigma$ & $\sigma$ & cps & $\sigma$ cps & $1 / T$ & \begin{tabular}{|c|} 
Cf $=$ \\
SQRT( ( \\
1/T)
\end{tabular} & $\begin{array}{c}\text { Corr. } \\
\text { cps }\end{array}$ & $\begin{array}{l}\text { Corr. } \\
\text { Sum } \\
\text { (cps) }\end{array}$ & \begin{tabular}{|c|} 
Drum \\
Activity \\
(dps)
\end{tabular} & \begin{tabular}{|c|} 
Drum \\
Activity \\
(nCi)
\end{tabular} & $\begin{array}{l}C_{f} \text { of } \\
\ln 1 / T\end{array}$ & $\begin{array}{l}\text { Corr. } \\
\text { Sum } \\
\text { (cps) }\end{array}$ & $\begin{array}{c}\text { Drum } \\
\text { Activity } \\
\text { (dps) }\end{array}$ & \begin{tabular}{|c|} 
Drum \\
Activity \\
(nCi)
\end{tabular} \\
\hline \multirow[t]{15}{*}{ FD562 } & T1562D1 & 300 & 3767 & 1.7 & 64.04 & 12.56 & 0.21 & 1.55 & 1.244 & & & & & 1.1809 & & & \\
\hline & T1562D2 & 300 & 1973 & 2.41 & 47.55 & 6.577 & 0.16 & & & & & & & & & & \\
\hline & T1562D3 & 300 & 1143 & 3.17 & 36.23 & 3.81 & \begin{tabular}{|l|}
0.12 \\
\end{tabular} & & & & & & & & & & \\
\hline & & & & & & & & & & & & & & & & & \\
\hline & T2562D1 & 300 & 1113 & 3.28 & 36.51 & 3.71 & \begin{tabular}{|l|}
0.12 \\
\end{tabular} & & & & & & & & & & \\
\hline & T2562D2 & 300 & 1556 & 2.75 & 42.79 & 5.187 & 0.14 & 11.03 & 3.321 & & & & & 2.222 & & & \\
\hline & T2562D3 & 300 & 1375 & 2.92 & 40.15 & 4.583 & 0.13 & & & & & & & & & & \\
\hline & & & & & & & & & & & & & & & & & \\
\hline & T3562D1 & 300 & 759 & 4.01 & 30.44 & 2.53 & 0.1 & & & & & & & & & & \\
\hline & T3562D2 & 300 & 1639 & 2.7 & 44.25 & 5.463 & 0.15 & & & & & & & & & & \\
\hline & T3562D3 & 300 & 930 & 3.62 & \begin{tabular}{|l|}
33.67 \\
\end{tabular} & 3.1 & \begin{tabular}{|l|}
0.11 \\
\end{tabular} & 56.29 & 7.502 & & & & & 3.3035 & & & \\
\hline & & & & & & & & & & & & & & & & & \\
\hline & 562D1 & 600 & 1165 & 3.26 & 37.98 & 1.942 & 0.06 & & & 2.41 & & & & 2.2929 & & & \\
\hline & 562D2 & 600 & 2219 & 2.31 & 51.26 & 3.698 & 0.09 & & & 12.28 & 35.77 & 76262 & 2059.1 & 8.2178 & 19.79 & 42192 & 1139.2 \\
\hline & 562D3 & 600 & 1685 & 2.64 & \begin{tabular}{|l|}
44.48 \\
\end{tabular} & 2.808 & 0.07 & & & 21.07 & & & & 9.2773 & & & \\
\hline & & & & & & & & & & & & & & & & & \\
\hline \multirow[t]{13}{*}{ FD554 } & T1554D1 & 300 & 1048 & 3.44 & 36.05 & 3.493 & 0.12 & 5.06 & 2.25 & & & & & 1.7684 & & & \\
\hline & T1554D2 & 300 & 339 & 7.7 & 26.1 & 1.13 & 0.09 & & & & & & & & & & \\
\hline & T1554D3 & 300 & 1521 & 2.97 & 45.17 & 5.07 & 0.15 & & & & & & & & & & \\
\hline & & & & & & & & & & & & & & & & & \\
\hline & T2554D1 & 300 & 125 & 15 & 18.75 & 0.417 & 0.06 & & & & & & & & & & \\
\hline & T2554D2 & 300 & 2576 & 2.15 & 55.38 & 8.587 & 0.18 & 2.03 & 1.426 & & & & & 1.3045 & & & \\
\hline & T2554D3 & 300 & 1405 & 3.13 & \begin{tabular}{|l|}
43.98 \\
\end{tabular} & 4.683 & 0.15 & & & & & & & & & & \\
\hline & & & & & & & & & & & & & & & & & \\
\hline & T3554D1 & 300 & 1134 & 3.33 & 37.76 & 3.78 & 0.13 & & & & & & & & & & \\
\hline & T3554D2 & 300 & 271 & 9.11 & 24.69 & 0.903 & 0.08 & & & & & & & & & & \\
\hline & T3554D3 & 300 & 1417 & 2.98 & 42.23 & 4.723 & 0.14 & 3.86 & 1.965 & & & & & 1.6222 & & & \\
\hline & & & & & & & & & & & & & & & & & \\
\hline & & & & & & & & & & & & & & & & & \\
\hline
\end{tabular}


WSRC-TR-2001-00004

Revision 0

January 8, 2001

Page 26 of 69

\begin{tabular}{|c|c|c|c|c|c|c|c|c|c|c|c|c|c|c|c|c|c|}
\hline Sample & File Name & $\begin{array}{l}\text { Count } \\
\text { Time }\end{array}$ & Area & $\% \sigma$ & $\sigma$ & cps & $\sigma$ cps & $1 / T$ & \begin{tabular}{|c|} 
Cf $=$ \\
SQRT( \\
1/T)
\end{tabular} & $\begin{array}{c}\text { Corr. } \\
\text { cps }\end{array}$ & $\begin{array}{l}\text { Corr. } \\
\text { Sum } \\
\text { (cps) }\end{array}$ & $\begin{array}{c}\text { Drum } \\
\text { Activity } \\
\text { (dps) }\end{array}$ & \begin{tabular}{|c|} 
Drum \\
Activity \\
(nCi)
\end{tabular} & $\begin{array}{l}C_{f} \text { of } \\
\ln 1 / T\end{array}$ & $\begin{array}{l}\text { Corr. } \\
\text { Sum } \\
\text { (cps) }\end{array}$ & \begin{tabular}{|c|} 
Drum \\
Activity \\
(dps)
\end{tabular} & $\begin{array}{c}\text { Drum } \\
\text { Activity } \\
\text { (nCi) }\end{array}$ \\
\hline & DF554D1 & 3600 & 907 & 5.81 & 52.7 & 0.252 & \begin{tabular}{|l|}
0.01 \\
\end{tabular} & & & 0.57 & & & & 0.4455 & & & \\
\hline & 554D2 & 3600 & 1862 & 3.91 & 72.8 & 0.517 & \begin{tabular}{|l|}
0.02 \\
\end{tabular} & & & 0.74 & 2.231 & 4756.9 & 128.44 & 0.6747 & 1.885 & 4019.1 & 108.52 \\
\hline & 554D3 & 3600 & 1697 & 4.41 & 74.84 & 0.471 & \begin{tabular}{|l|}
0.02 \\
\end{tabular} & & & 0.93 & & & & 0.7647 & & & \\
\hline & & & & & & & & & & & & & & & & & \\
\hline \multirow[t]{15}{*}{ FD559 } & T1559D1 & 335.22 & 4083 & 1.63 & 66.55 & 12.18 & 0.2 & 1.36 & \begin{tabular}{l|}
1.166 \\
\end{tabular} & & & & & 1.1255 & & & \\
\hline & T1559D2 & 335.22 & 816 & 3.85 & 31.42 & 2.434 & \begin{tabular}{|l|}
0.09 \\
\end{tabular} & & & & & & & & & & \\
\hline & T1559D3 & 335.22 & 682 & 4.11 & 28.03 & 2.034 & \begin{tabular}{|l|}
0.08 \\
\end{tabular} & & & & & & & & & & \\
\hline & & & & & & & & & & & & & & & & & \\
\hline & T2559D1 & 346.05 & 908 & 3.58 & 32.51 & 2.624 & \begin{tabular}{|l|}
0.09 \\
\end{tabular} & & & & & & & & & & \\
\hline & T2559D2 & 346.05 & 1327 & 2.97 & 39.41 & 3.835 & \begin{tabular}{|l|}
0.11 \\
\end{tabular} & 4.46 & 2.112 & & & & & 1.6993 & & & \\
\hline & T2559D3 & 346.05 & 952 & 3.53 & 33.61 & 2.751 & 0.1 & & & & & & & & & & \\
\hline & & & & & & & & & & & & & & & & & \\
\hline & T3559D1 & 600 & 661 & 4.33 & 28.62 & 1.102 & \begin{tabular}{|l|}
0.05 \\
\end{tabular} & & & & & & & & & & \\
\hline & T3559D2 & 600 & 1481 & 2.79 & 41.32 & 2.468 & \begin{tabular}{|l|}
0.07 \\
\end{tabular} & & & & & & & & & & \\
\hline & T3559D3 & 600 & 176 & 9.39 & 16.53 & 0.293 & \begin{tabular}{|l|}
0.03 \\
\end{tabular} & 81.95 & 9.052 & & & & & 3.5711 & & & \\
\hline & & & & & & & & & & & & & & & & & \\
\hline & 559D1 & 1000 & 108 & 12.03 & 12.99 & 0.108 & \begin{tabular}{|l|}
0.01 \\
\end{tabular} & & & 0.13 & & & & 0.1216 & & & \\
\hline & 559D2 & 1000 & 155 & 9.92 & 15.38 & 0.155 & \begin{tabular}{|l|}
0.02 \\
\end{tabular} & & & 0.33 & 1.295 & 2761.6 & 74.564 & 0.2634 & 0.717 & 1528.9 & 41.28 \\
\hline & 559D3 & 1000 & 93 & 14.13 & 13.14 & 0.093 & \begin{tabular}{|l|}
0.01 \\
\end{tabular} & & & 0.84 & & & & 0.3321 & & & \\
\hline & & & & & & & & & & & & & & & & & \\
\hline \multirow[t]{9}{*}{ FD563 } & T1563D1 & 271.01 & 2817 & 1.96 & 55.21 & 10.39 & 0.2 & 3.11 & 1.763 & & & & & & & & \\
\hline & T1563D2 & 271.01 & 647 & 4.31 & 27.89 & 2.387 & 0.1 & & & & & & & & & & \\
\hline & T1563D3 & 271.01 & 169 & 8.81 & 14.89 & 0.624 & \begin{tabular}{|l|}
0.05 \\
\end{tabular} & & & & & & & & & & \\
\hline & & & & & & & & & & & & & & & & & \\
\hline & T2563D1 & 463.04 & 1110 & 3.29 & 36.52 & 2.397 & \begin{tabular}{|l|}
0.08 \\
\end{tabular} & & & & & & & & & & \\
\hline & T2563D2 & 463.04 & 458 & 5.21 & 23.86 & 0.989 & \begin{tabular}{|l|}
0.05 \\
\end{tabular} & -6.47 & $\mathrm{~N} / \mathrm{A}$ & & & & & & & & \\
\hline & T2563D3 & 463.04 & 866 & 3.72 & 32.22 & 1.87 & \begin{tabular}{|l|}
0.07 \\
\end{tabular} & & & & & & & & & & \\
\hline & & & & & & & & & & & & & & & & & \\
\hline & & & & & & & & & & & & & & & & & \\
\hline
\end{tabular}


WSRC-TR-2001-00004

Revision 0

January 8, 2001

Page 27 of 69

\begin{tabular}{|c|c|c|c|c|c|c|c|c|c|c|c|c|c|c|c|c|c|}
\hline Sample & File Name & $\begin{array}{l}\text { Count } \\
\text { Time }\end{array}$ & Area & $\% \sigma$ & $\sigma$ & cps & $\sigma$ cps & $1 / \mathbf{T}$ & $\begin{array}{c}\text { Cf }= \\
\text { SQRT( }( \\
\text { 1/T) }\end{array}$ & $\begin{array}{c}\text { Corr. } \\
\text { cps }\end{array}$ & $\begin{array}{l}\text { Corr. } \\
\text { Sum } \\
\text { (cps) }\end{array}$ & \begin{tabular}{|c|} 
Drum \\
Activity \\
(dps)
\end{tabular} & \begin{tabular}{|c|} 
Drum \\
Activity \\
(nCi)
\end{tabular} & $\begin{array}{l}C_{f} \text { of } \\
\ln 1 / T\end{array}$ & $\begin{array}{l}\text { Corr. } \\
\text { Sum } \\
\text { (cps) }\end{array}$ & $\begin{array}{c}\text { Drum } \\
\text { Activity } \\
\text { (dps) }\end{array}$ & \begin{tabular}{|c|} 
Drum \\
Activity \\
(nCi)
\end{tabular} \\
\hline & T3563D1 & 300 & 277 & 6.75 & \begin{tabular}{ll|}
18.7 \\
\end{tabular} & 0.923 & 0.06 & & & & & & & & & & \\
\hline & T3563D2 & 300 & 349 & 6.19 & 21.6 & 1.163 & 0.07 & & & & & & & & & & \\
\hline & T3563D3 & 300 & 796 & 3.72 & 29.61 & 2.653 & 0.1 & 13.05 & 3.612 & & & & & & & & \\
\hline & & & & & & & & & & & & & & & & & \\
\hline & 563D1 & 3600 & 18400 & 0.82 & 150.9 & 5.111 & 0.04 & & & 9.01 & & & & & & & \\
\hline & 563D2 & 3600 & 12692 & 1.05 & 133.3 & 3.526 & 0.04 & & & $\mathrm{~N} / \mathrm{A}$ & $\mathrm{N} / \mathrm{A}$ & $\mathrm{N} / \mathrm{A}$ & $\mathrm{N} / \mathrm{A}$ & & & & \\
\hline & 563D3 & 3600 & 5022 & 1.82 & 91.4 & 1.395 & 0.03 & & & 5.04 & & & & & & & \\
\hline & & & & & & & & & & & & & & & & & \\
\hline \multirow[t]{16}{*}{ EMTY DRM } & T1EDD1 & 300 & 3115 & 1.86 & 57.94 & 10.38 & 0.19 & 1.58 & 1.258 & & & & & 1.1907 & & & \\
\hline & T1EDD2 & 300 & 3356 & 1.79 & 60.07 & 11.19 & 0.2 & & & & & & & & & & \\
\hline & T1EDD3 & 300 & 2431 & 2.11 & 51.29 & 8.103 & 0.17 & & & & & & & & & & \\
\hline & & & & & & & & & & & & & & & & & \\
\hline & T2EDD1 & 300 & 2804 & 1.97 & 55.24 & 9.347 & 0.18 & & & & & & & & & & \\
\hline & T2EDD2 & 300 & 3420 & 1.78 & 60.88 & 11.4 & 0.2 & 1.44 & 1.201 & & & & & 1.151 & & & \\
\hline & T2EDD3 & 300 & 3189 & 1.85 & 59 & 10.63 & 0.2 & & & & & & & & & & \\
\hline & & & & & & & & & & & & & & & & & \\
\hline & T3EDD1 & 300 & 2069 & 2.31 & 47.79 & 6.897 & 0.16 & & & & & & & & & & \\
\hline & T3EDD2 & 300 & 3046 & 1.89 & 57.57 & 10.15 & 0.19 & & & & & & & & & & \\
\hline & T3EDD3 & 300 & 2061 & 2.28 & 46.99 & 6.87 & 0.16 & 2.40 & 1.548 & & & & & 1.3818 & & & \\
\hline & & & & & & & & & & & & & & & & & \\
\hline & EPDD1 & 300 & 1 & 500 & 5 & 0.003 & \begin{tabular}{|l|}
0.02 \\
\end{tabular} & & & 0.00 & & & & 0.004 & & & \\
\hline & EPDD2 & 300 & 8 & 61 & 4.88 & 0.027 & \begin{tabular}{|l|}
0.02 \\
\end{tabular} & & & 0.03 & 0.062 & 132.25 & 3.5708 & 0.0307 & 0.058 & 123.01 & 3.3212 \\
\hline & EPDD3 & 300 & 5 & 89 & \begin{tabular}{l|l|}
4.45 \\
\end{tabular} & 0.017 & 0.01 & & & 0.03 & & & & 0.023 & & & \\
\hline & & & & & 0 & & & & & & & & & & & & \\
\hline \multirow[t]{5}{*}{ FD565 } & T1565D1 & 300 & 1090 & 3.65 & 39.79 & 3.633 & 0.13 & 24.28 & 4.927 & & & & & 2.7267 & & & \\
\hline & T1565D2 & 300 & 2425 & 2.31 & 56.02 & 8.083 & 0.19 & & & & & & & & & & \\
\hline & T1565D3 & 300 & 1040 & 3.68 & 38.27 & 3.467 & 0.13 & & & & & & & & & & \\
\hline & & & & & & & & & & & & & & & & & \\
\hline & & & & & & & & & & & & & & & & & \\
\hline
\end{tabular}


WSRC-TR-2001-00004

Revision 0

January 8, 2001

Page 28 of 69

\begin{tabular}{|c|c|c|c|c|c|c|c|c|c|c|c|c|c|c|c|c|c|}
\hline Sample & File Name & $\begin{array}{l}\text { Count } \\
\text { Time }\end{array}$ & Area & $\% \sigma$ & $\sigma$ & cps & $\sigma$ cps & $1 / T$ & $\begin{array}{c}\text { Cf }= \\
\text { SQRT( }( \\
\text { 1/T) }\end{array}$ & $\begin{array}{c}\text { Corr. } \\
\text { cps }\end{array}$ & $\begin{array}{l}\text { Corr. } \\
\text { Sum } \\
\text { (cps) }\end{array}$ & \begin{tabular}{|c|} 
Drum \\
Activity \\
(dps)
\end{tabular} & \begin{tabular}{|c|} 
Drum \\
Activity \\
(nCi)
\end{tabular} & $\begin{array}{l}C_{f} \text { of } \\
\ln 1 / T\end{array}$ & $\begin{array}{l}\text { Corr. } \\
\text { Sum } \\
\text { (cps) }\end{array}$ & $\begin{array}{c}\text { Drum } \\
\text { Activity } \\
\text { (dps) }\end{array}$ & \begin{tabular}{|c|} 
Drum \\
Activity \\
(nCi)
\end{tabular} \\
\hline & T2565D1 & 300 & 1312 & 3.27 & 42.9 & 4.373 & 0.14 & & & & & & & & & & \\
\hline & T2565D2 & 300 & 2224 & 2.46 & 54.71 & 7.413 & 0.18 & 6.83 & 2.614 & & & & & 1.9372 & & & \\
\hline & T2565D3 & 300 & 1883 & 2.6 & 48.96 & 6.277 & 0.16 & & & & & & & & & & \\
\hline & & & & & & & & & & & & & & & & & \\
\hline & T3565D1 & 300 & 3392 & 1.88 & 63.77 & 11.31 & 0.21 & & & & & & & & & & \\
\hline & T3565D2 & 300 & 2323 & 2.36 & 54.82 & 7.743 & 0.18 & & & & & & & & & & \\
\hline & T3565D3 & 300 & 984 & 3.8 & 37.39 & 3.28 & 0.12 & 16.68 & 4.085 & & & & & 2.4818 & & & \\
\hline & & & & & & & & & & & & & & & & & \\
\hline & 565D1 & \begin{tabular}{|c|}
661.13 \\
\end{tabular} & 1955 & 2.69 & 52.59 & 2.957 & 0.08 & & & 14.57 & & & & 8.063 & & & \\
\hline & 565D2 & 661.13 & 3313 & 2.69 & 89.12 & 5.011 & 0.13 & & & 13.10 & 37.05 & 78994 & 2132.8 & 9.7075 & 23.47 & 50040 & 1351.1 \\
\hline & 565D3 & 661.13 & 1518 & 3.13 & 47.51 & 2.296 & \begin{tabular}{|l|}
0.07 \\
\end{tabular} & & & 9.38 & & & & 5.6984 & & & \\
\hline 0.000469 & 0.027 & & & & & & & & & & & & & & & & \\
\hline & & & & & & & & & & & & & & & & & \\
\hline & & & & & & & & & & & & & & & & & \\
\hline T01 & T1DET1 & 101.87 & 1665 & 2.51 & 41.79 & 16.34 & 0.41 & & & & & & & & & & \\
\hline & T1det2 & 101.87 & 1617 & 2.55 & 41.23 & 15.87 & 0.4 & & & & & & & & & & \\
\hline & T1det3 & 101.87 & 1115 & 3.1 & 34.57 & 10.95 & 0.34 & & & & & & & & & & \\
\hline & & & & & & & & & & & & & & & & & \\
\hline T02 & T2det1 & 100 & 1432 & 2.7 & 38.66 & 14.32 & 0.39 & & & & & & & & & & \\
\hline & T2det2 & 100 & 1715 & 2.47 & 42.36 & 17.15 & 0.42 & & & & & & & & & & \\
\hline & T2det3 & 100 & 1623 & 2.55 & 41.39 & 16.23 & 0.41 & & & & & & & & & & \\
\hline & & & & & & & & & & & & & & & & & \\
\hline T03 & T3det1 & 100 & 1112 & 3.06 & 34.03 & 11.12 & 0.34 & & & & & & & & & & \\
\hline & T3det2 & 100 & 1576 & 2.59 & 40.82 & 15.76 & 0.41 & & & & & & & & & & \\
\hline & T3det3 & 100 & 1714 & 2.49 & 42.68 & 17.14 & 0.43 & & & & & & & & & & \\
\hline & & & & & & & & 16.88 & & & & & & & & & \\
\hline FD570 & T1570D1 & 271.45 & 2482 & 2.13 & 52.87 & 9.143 & 0.19 & 2.48 & 1.574 & & & & & $\begin{array}{l}1.3981 \\
\end{array}$ & & & \\
\hline & T1570D2 & 271.45 & 1932 & 2.44 & 47.14 & 7.117 & 0.17 & & & & & & & & & & \\
\hline & T1570D3 & 271.45 & 906 & 3.55 & 32.16 & 3.338 & 0.12 & & & & & & & & & & \\
\hline
\end{tabular}


WSRC-TR-2001-00004

Revision 0

January 8, 2001

Page 29 of 69

\begin{tabular}{|c|c|c|c|c|c|c|c|c|c|c|c|c|c|c|c|c|c|}
\hline Sample & File Name & $\begin{array}{l}\text { Count } \\
\text { Time }\end{array}$ & Area & $\% \sigma$ & $\sigma$ & cps & $\sigma$ cps & $1 / T$ & $\begin{array}{c}\text { Cf }= \\
\text { SQRT( }( \\
\text { 1/T) }\end{array}$ & $\begin{array}{c}\text { Corr. } \\
\text { cps }\end{array}$ & $\begin{array}{l}\text { Corr. } \\
\text { Sum } \\
\text { (cps) }\end{array}$ & \begin{tabular}{|c|} 
Drum \\
Activity \\
(dps)
\end{tabular} & \begin{tabular}{|c|} 
Drum \\
Activity \\
(nCi)
\end{tabular} & $\begin{array}{l}C_{f} \text { of } \\
\ln 1 / T\end{array}$ & $\begin{array}{l}\text { Corr. } \\
\text { Sum } \\
\text { (cps) }\end{array}$ & $\begin{array}{c}\text { Drum } \\
\text { Activity } \\
\text { (dps) }\end{array}$ & \begin{tabular}{|c|} 
Drum \\
Activity \\
(nCi)
\end{tabular} \\
\hline & T2570D1 & 300 & 1473 & 2.79 & 41.1 & 4.91 & \begin{tabular}{|l|}
0.14 \\
\end{tabular} & & & & & & & & & & \\
\hline & T2570D2 & 300 & 1836 & 2.5 & 45.9 & 6.12 & 0.15 & 5.36 & 2.316 & & & & & 1.7999 & & & \\
\hline & T2570D3 & 300 & 1460 & 2.86 & 41.76 & 4.867 & 0.14 & & & & & & & & & & \\
\hline & & & & & & & & & & & & & & & & & \\
\hline & T3570D2 & 300 & 1113 & 3.21 & 35.73 & 3.71 & 0.12 & & & & & & & & & & \\
\hline & T3570D2 & 300 & 1864 & 2.5 & 46.6 & 6.213 & 0.16 & & & & & & & & & & \\
\hline & T3570D3 & 300 & 1105 & 3.22 & 35.58 & 3.683 & 0.12 & 7.42 & 2.724 & & & & & 1.9851 & & & \\
\hline & & & & & & & & & & & & & & & & & \\
\hline & 570D1 & \begin{tabular}{l|}
1549.2 \\
\end{tabular} & 3614 & 1.82 & 65.77 & 2.333 & 0.04 & & & 3.67 & & & & 3.2615 & & & \\
\hline & 570D2 & 1549.2 & 4738 & 1.6 & 75.81 & 3.058 & 0.05 & & & 7.08 & 14.76 & 31479 & 849.93 & 5.5047 & 11.69 & 24920 & 672.85 \\
\hline & 570D3 & \begin{tabular}{l|l}
1549.2 \\
\end{tabular} & 2280 & 2.33 & 53.12 & 1.472 & 0.03 & & & 4.01 & & & & 2.9214 & & & \\
\hline & & & & & & & & & & & & & & & & & \\
\hline \multirow[t]{17}{*}{\begin{tabular}{|l|} 
FD564 \\
\end{tabular}} & T1564D1 & 166.17 & 1388 & 2.84 & 39.42 & 8.353 & 0.24 & 2.15 & 1.467 & & & & & 1.3307 & & & \\
\hline & T1564D2 & 166.17 & 410 & 5.56 & 22.8 & 2.467 & 0.14 & & & & & & & & & & \\
\hline & T1564D3 & 166.17 & 311 & 6.21 & 19.31 & 1.872 & 0.12 & & & & & & & & & & \\
\hline & & & & & & & & & & & & & & & & & \\
\hline & T2564D1 & 300 & 456 & 5.28 & 24.08 & 1.52 & 0.08 & & & & & & & & & & \\
\hline & \begin{tabular}{|l|} 
T2564D2 \\
\end{tabular} & 300 & 748 & 4.01 & 29.99 & 2.493 & 0.1 & 11.29 & 3.36 & & & & & 2.2362 & & & \\
\hline & T2564D3 & 300 & 785 & 3.96 & 31.09 & 2.617 & 0.1 & & & & & & & & & & \\
\hline & & & & & & & & & & & & & & & & & \\
\hline & T3564D1 & 300 & 338 & 6.24 & 21.09 & 1.127 & \begin{tabular}{|l|}
0.07 \\
\end{tabular} & & & & & & & & & & \\
\hline & T3564D2 & 300 & 725 & 4.09 & 29.65 & 2.417 & 0.1 & & & & & & & & & & \\
\hline & T3564D3 & 300 & 1077 & 3.31 & 35.65 & 3.59 & \begin{tabular}{|l|}
0.12 \\
\end{tabular} & 6.12 & 2.473 & & & & & 1.874 & & & \\
\hline & & & & & & & & & & & & & & & & & \\
\hline & 564D1 & 900 & 457 & 5.34 & 24.4 & 0.508 & 0.03 & & & 0.74 & & & & 0.6757 & & & \\
\hline & 564D2 & 900 & 935 & 3.67 & 34.31 & 1.039 & \begin{tabular}{|l|} 
\\
\end{tabular} & & & 3.49 & 6.478 & 13812 & 372.92 & 2.3231 & 4.698 & 10017 & 270.45 \\
\hline & 564D3 & 900 & 816 & 3.97 & 32.4 & 0.907 & \begin{tabular}{|l|}
0.04 \\
\end{tabular} & & & 2.24 & & & & 1.6991 & & & \\
\hline & & & & & & & & & & & & & & & & & \\
\hline & & & & & & & & & & & & & & & & & \\
\hline
\end{tabular}


WSRC-TR-2001-00004

Revision 0

January 8, 2001

Page 30 of 69

\begin{tabular}{|c|c|c|c|c|c|c|c|c|c|c|c|c|c|c|c|c|c|}
\hline Sample & File Name & \begin{tabular}{c|} 
Count \\
Time
\end{tabular} & Area & $\% \sigma$ & $\sigma$ & cps & $\sigma$ cps & $1 / \mathbf{T}$ & \begin{tabular}{|c|} 
Cf $=$ \\
SQRT( \\
1/T)
\end{tabular} & $\begin{array}{c}\text { Corr. } \\
\text { cps }\end{array}$ & $\begin{array}{l}\text { Corr. } \\
\text { Sum } \\
\text { (cps) }\end{array}$ & \begin{tabular}{|c|} 
Drum \\
Activity \\
(dps)
\end{tabular} & $\begin{array}{c}\text { Drum } \\
\text { Activity } \\
\text { (nCi) }\end{array}$ & $\begin{array}{l}C_{f} \text { of } \\
\ln 1 / T\end{array}$ & $\begin{array}{l}\text { Corr. } \\
\text { Sum } \\
\text { (cps) }\end{array}$ & $\begin{array}{c}\text { Drum } \\
\text { Activity } \\
\text { (dps) }\end{array}$ & $\begin{array}{c}\text { Drum } \\
\text { Activity } \\
\text { (nCi) }\end{array}$ \\
\hline \multirow[t]{15}{*}{\begin{tabular}{|l|} 
FD555 \\
\end{tabular}} & T1555D1 & 300 & 3432 & 1.78 & 61.09 & 11.44 & 0.2 & 1.48 & 1.216 & & & & & 1.1611 & & & \\
\hline & T1555D2 & 300 & 251 & 7.64 & 19.18 & 0.837 & 0.06 & & & & & & & & & & \\
\hline & T1555D3 & 300 & 466 & 5.08 & 23.67 & 1.553 & 0.08 & & & & & & & & & & \\
\hline & & & & & & & & & & & & & & & & & \\
\hline & T2555D1 & 300 & 217 & 6.73 & 14.6 & 0.723 & 0.05 & & & & & & & & & & \\
\hline & T2555D2 & 300 & 1371 & 2.9 & 39.76 & 4.57 & 0.13 & 3.62 & 1.901 & & & & & $\begin{array}{l}1.5878 \\
\end{array}$ & & & \\
\hline & T2555D3 & 300 & 489 & 5.05 & 24.69 & 1.63 & 0.08 & & & & & & & & & & \\
\hline & & & & & & & & & & & & & & & & & \\
\hline & T3555D1 & 300 & 281 & 6.66 & 18.71 & 0.937 & 0.06 & & & & & & & & & & \\
\hline & T3555D2 & 300 & 957 & 3.46 & 33.11 & 3.19 & 0.11 & & & & & & & & & & \\
\hline & T3555d2 & 300 & 169 & 9.4 & 15.89 & 0.563 & 0.05 & 30.21 & 5.497 & & & & & 2.8734 & & & \\
\hline & & & & & & & & & & & & & & & & & \\
\hline & 555d1 & 3600 & 70 & 28 & 19.6 & 0.019 & 0.01 & & & 0.02 & & & & $\begin{array}{ll}0.0226 \\
\end{array}$ & & & \\
\hline & $555 d 2$ & 3600 & 104 & 23 & 23.92 & 0.029 & 0.01 & & & 0.05 & 0.189 & 401.92 & 10.852 & 0.0459 & 0.126 & 268.48 & 7.2489 \\
\hline & $555 d 3$ & 3600 & 72 & 29 & 20.88 & 0.02 & 0.01 & & & 0.11 & & & & 0.0575 & & & \\
\hline & & & & & & & & & & & & & & & & & \\
\hline \multirow[t]{13}{*}{\begin{tabular}{|l|} 
FD419 \\
\end{tabular}} & T1419D1 & 136.32 & 1846 & 2.41 & 44.49 & 13.54 & 0.33 & 1.30 & 1.138 & & & & & 1.1052 & & & \\
\hline & T1419D2 & 136.32 & 779 & 3.92 & 30.54 & 5.714 & 0.22 & & & & & & & & & & \\
\hline & T1419D3 & 136.32 & 590 & 4.56 & 26.9 & 4.328 & 0.2 & & & & & & & & & & \\
\hline & & & & & & & & & & & & & & & & & \\
\hline & T2419D1 & 300 & 1642 & 2.61 & 42.86 & 5.473 & 0.14 & & & & & & & & & & \\
\hline & \begin{tabular}{|l|} 
T2419D2 \\
\end{tabular} & 300 & 1809 & 2.55 & 46.13 & 6.03 & 0.15 & 3.72 & 1.929 & & & & & 1.6028 & & & \\
\hline & T2419D3 & 300 & 1373 & 2.96 & 40.64 & 4.577 & 0.14 & & & & & & & & & & \\
\hline & & & & & & & & & & & & & & & & & \\
\hline & T3419D1 & 300 & 812 & 3.91 & 31.75 & 2.707 & 0.11 & & & & & & & & & & \\
\hline & T3419D2 & 300 & 932 & 3.64 & 33.92 & 3.107 & 0.11 & & & & & & & & & & \\
\hline & T3419D3 & 300 & 912 & 3.72 & 33.93 & 3.04 & 0.11 & 19.70 & 4.439 & & & & & 2.5893 & & & \\
\hline & & & & & & & & & & & & & & & & & \\
\hline & & & & & & & & & & & & & & & & & \\
\hline
\end{tabular}


WSRC-TR-2001-00004

Revision 0

January 8, 2001

Page 31 of 69

\begin{tabular}{|c|c|c|c|c|c|c|c|c|c|c|c|c|c|c|c|c|c|}
\hline Sample & File Name & \begin{tabular}{c|} 
Count \\
Time
\end{tabular} & Area & $\% \sigma$ & $\sigma$ & cps & $\sigma$ cps & $1 / \mathbf{T}$ & $\begin{array}{c}\text { Cf }= \\
\text { SQRT( }( \\
\text { 1/T) }\end{array}$ & $\begin{array}{c}\text { Corr. } \\
\text { cps }\end{array}$ & $\begin{array}{l}\text { Corr. } \\
\text { Sum } \\
\text { (cps) }\end{array}$ & \begin{tabular}{|c|} 
Drum \\
Activity \\
(dps)
\end{tabular} & $\begin{array}{c}\text { Drum } \\
\text { Activity } \\
\text { (nCi) }\end{array}$ & $\begin{array}{l}C_{f} \text { of } \\
\ln 1 / T\end{array}$ & $\begin{array}{l}\text { Corr. } \\
\text { Sum } \\
\text { (cps) }\end{array}$ & $\begin{array}{c}\text { Drum } \\
\text { Activity } \\
\text { (dps) }\end{array}$ & $\begin{array}{c}\text { Drum } \\
\text { Activity } \\
\text { (nCi) }\end{array}$ \\
\hline & 419D1 & 605.41 & 313 & 6.44 & 20.16 & 0.517 & 0.03 & & & 0.59 & & & & 0.5714 & & & \\
\hline & 419D2 & 605.41 & 979 & 3.56 & 34.85 & 1.617 & 0.06 & & & 3.12 & 13.5 & 28791 & 777.35 & 2.5918 & 8.877 & 18928 & 511.06 \\
\hline & 419D3 & 605.41 & 1336 & 3.01 & 40.21 & 2.207 & \begin{tabular}{|l|}
0.07 \\
\end{tabular} & & & 9.80 & & & & 5.7141 & & & \\
\hline & & & & & & & & & & & & & & & & & \\
\hline \multirow[t]{17}{*}{ FD572 } & T1572d1 & 300 & 1908 & 2.4 & 45.79 & 6.36 & 0.15 & 2.92 & 1.71 & & & & & 1.4796 & & & \\
\hline & T1572D2 & 300 & 2069 & 2.35 & 48.62 & 6.897 & 0.16 & & & & & & & & & & \\
\hline & T1572D3 & 300 & 2269 & 2.24 & 50.83 & 7.563 & 0.17 & & & & & & & & & & \\
\hline & & & & & & & & & & & & & & & & & \\
\hline & T2572D1 & 250.4 & 1360 & 2.88 & 39.17 & 5.431 & \begin{tabular}{|l|}
0.16 \\
\end{tabular} & & & & & & & & & & \\
\hline & T2572D2 & 250.4 & 2147 & 2.27 & 48.74 & 8.574 & 0.19 & 2.28 & 1.511 & & & & & 1.3587 & & & \\
\hline & T2572D3 & 250.4 & 1254 & 3.01 & 37.75 & 5.008 & 0.15 & & & & & & & & & & \\
\hline & & & & & & & & & & & & & & & & & \\
\hline & T3572D1 & 300 & 1488 & 2.76 & 41.07 & 4.96 & 0.14 & & & & & & & & & & \\
\hline & T3572D2 & 300 & 947 & 3.56 & 33.71 & 3.157 & 0.11 & & & & & & & & & & \\
\hline & T3672D3 & 300 & 1064 & 3.34 & 35.54 & 3.547 & 0.12 & 52.24 & 7.228 & & & & & 3.2511 & & & \\
\hline & & & & & & & & & & & & & & & & & \\
\hline & $572 d 1$ & 370.31 & 218 & 7.84 & 17.09 & 0.589 & 0.05 & & & 1.01 & & & & 0.8711 & & & \\
\hline & 572D2 & 370.31 & 512 & 4.94 & 25.29 & 1.383 & 0.07 & & & 2.09 & 26.46 & 56417 & 1523.2 & 1.8786 & 13.26 & 28270 & 763.28 \\
\hline & 572D3 & 370.31 & 1197 & 3.08 & 36.87 & 3.232 & 0.1 & & & 23.36 & & & & 10.509 & & & \\
\hline & & & & & & & & & & & & & & & & & \\
\hline & & & \#\#\#\#\# & & & & & & & & & & & & & & \\
\hline \multirow[t]{8}{*}{ T0 } & T01D1 & 100 & 1640 & 2.53 & 41.49 & 16.4 & 0.41 & & & & & & & & & & \\
\hline & T01D2 & 100 & 1503 & 2.68 & 40.28 & 15.03 & \begin{tabular}{|l|} 
\\
\end{tabular} & & & & & & & & & & \\
\hline & T01D3 & 100 & 1284 & 2.86 & 36.72 & 12.84 & 0.37 & & & & & & & & & & \\
\hline & & & & & & & & & & & & & & & & & \\
\hline & T02D1 & 100 & 1385 & 2.76 & 38.23 & 13.85 & 0.38 & & & & & & & & & & \\
\hline & T02D2 & 100 & 1653 & 2.54 & 41.99 & 16.53 & \begin{tabular}{|l|} 
\\
\end{tabular} & & & & & & & & & & \\
\hline & T02D3 & 100 & 1557 & 2.63 & 40.95 & 15.57 & 0.41 & & & & & & & & & & \\
\hline & & & & & & & & & & & & & & & & & \\
\hline
\end{tabular}


WSRC-TR-2001-00004

Revision 0

January 8, 2001

Page 32 of 69

\begin{tabular}{|c|c|c|c|c|c|c|c|c|c|c|c|c|c|c|c|c|c|}
\hline Sample & File Name & \begin{tabular}{c|} 
Count \\
Time
\end{tabular} & Area & $\% \sigma$ & $\sigma$ & cps & $\sigma$ cps & $1 / T$ & $\begin{array}{c}\text { Cf }= \\
\text { SQRT( }( \\
\text { 1/T) }\end{array}$ & $\begin{array}{c}\text { Corr. } \\
\text { cps }\end{array}$ & $\begin{array}{l}\text { Corr. } \\
\text { Sum } \\
\text { (cps) }\end{array}$ & \begin{tabular}{|c|} 
Drum \\
Activity \\
(dps)
\end{tabular} & $\begin{array}{c}\text { Drum } \\
\text { Activity } \\
\text { (nCi) }\end{array}$ & $\begin{array}{l}C_{f} \text { of } \\
\ln 1 / T\end{array}$ & $\begin{array}{l}\text { Corr. } \\
\text { Sum } \\
\text { (cps) }\end{array}$ & $\begin{array}{c}\text { Drum } \\
\text { Activity } \\
\text { (dps) }\end{array}$ & $\begin{array}{c}\text { Drum } \\
\text { Activity } \\
\text { (nCi) }\end{array}$ \\
\hline & T03D1 & 100 & 1024 & 3.22 & 32.97 & 10.24 & 0.33 & & & & & & & & & & \\
\hline & T03dD2 & 100 & 1449 & 2.71 & 39.27 & 14.49 & 0.39 & & & & & & & & & & \\
\hline & T03D3 & 100 & 1591 & 2.59 & 41.21 & 15.91 & 0.41 & & & & & & & & & & \\
\hline & & & & & & & & 16.28 & & & & & & & & & \\
\hline \multirow[t]{15}{*}{ FD412 } & T1412D1 & 300 & 2937 & 1.92 & 56.39 & 9.79 & 0.19 & 1.67 & 1.29 & & & & & 1.2135 & & & \\
\hline & T1412D2 & 300 & 424 & 5.69 & 24.13 & 1.413 & 0.08 & & & & & & & & & & \\
\hline & T1412D3 & 300 & 622 & 4.36 & 27.12 & 2.073 & 0.09 & & & & & & & & & & \\
\hline & & & & & & & & & & & & & & & & & \\
\hline & T2412D1 & 300 & 435 & 5.21 & 22.66 & 1.45 & 0.08 & & & & & & & & & & \\
\hline & T2412D2 & 300 & 916 & 3.69 & 33.8 & 3.053 & 0.11 & 5.41 & 2.326 & & & & & 1.8051 & & & \\
\hline & T2412D3 & 300 & 784 & 3.91 & 30.65 & 2.613 & 0.1 & & & & & & & & & & \\
\hline & & & & & & & & & & & & & & & & & \\
\hline & T3412D1 & 300 & 525 & 4.82 & 25.31 & 1.75 & 0.08 & & & & & & & & & & \\
\hline & T3412D2 & 300 & 763 & 3.56 & 27.16 & 2.543 & 0.09 & & & & & & & & & & \\
\hline & T3412D3 & 300 & 1069 & 3.28 & 35.06 & 3.563 & 0.12 & 4.57 & 2.137 & & & & & 1.7121 & & & \\
\hline & & & & & & & & & & & & & & & & & \\
\hline & 412D1 & 6000 & 85 & 119 & 101.2 & 0.014 & \begin{tabular}{|l|} 
\\
\end{tabular} & & & 0.02 & & & & 0.0172 & & & \\
\hline & 412D2 & 6000 & 273 & 50 & 136.5 & 0.046 & 0.02 & & & 0.11 & 0.124 & 264.68 & 7.1465 & 0.0821 & 0.099 & 211.78 & 5.7181 \\
\hline & 412D3 & 6000 & 0 & 0 & 0 & 0 & 0 & & & 0.00 & & & & 0 & & & \\
\hline & & & & & & & & & & & & & & & & & \\
\hline \multirow[t]{9}{*}{ FD567 } & T1567D1 & 300 & 3137 & 1.86 & 58.35 & 10.46 & 0.19 & 1.58 & 1.255 & & & & & 1.1891 & & & \\
\hline & T1567D2 & 300 & 633 & 4.39 & 27.79 & 2.11 & \begin{tabular}{|l|}
0.09 \\
\end{tabular} & & & & & & & & & & \\
\hline & T1567D3 & 300 & 60 & 20.56 & 12.34 & 0.2 & 0.04 & & & & & & & & & & \\
\hline & & & & & & & & & & & & & & & & & \\
\hline & T2567D1 & 300 & 542 & 4.7 & 25.47 & 1.807 & 0.08 & & & & & & & & & & \\
\hline & \begin{tabular}{|l|} 
T2567D2 \\
\end{tabular} & 300 & 153 & 9.86 & 15.09 & 0.51 & \begin{tabular}{|l|}
0.05 \\
\end{tabular} & 46.96 & 6.853 & & & & & 3.1765 & & & \\
\hline & T2567D3 & 300 & 545 & 4.82 & 26.27 & 1.817 & \begin{tabular}{|l|}
0.09 \\
\end{tabular} & & & & & & & & & & \\
\hline & & & & & & & & & & & & & & & & & \\
\hline & & & & & & & & & & & & & & & & & \\
\hline
\end{tabular}


WSRC-TR-2001-00004

Revision 0

January 8, 2001

Page 33 of 69

\begin{tabular}{|c|c|c|c|c|c|c|c|c|c|c|c|c|c|c|c|c|c|}
\hline Sample & File Name & \begin{tabular}{c|} 
Count \\
Time
\end{tabular} & Area & $\% \sigma$ & $\sigma$ & cps & $\sigma$ cps & $1 / T$ & $\begin{array}{c}\text { Cf }= \\
\text { SQRT( }( \\
\text { 1/T) }\end{array}$ & $\begin{array}{c}\text { Corr. } \\
\text { cps }\end{array}$ & $\begin{array}{l}\text { Corr. } \\
\text { Sum } \\
\text { (cps) }\end{array}$ & \begin{tabular}{|c|} 
Drum \\
Activity \\
(dps)
\end{tabular} & \begin{tabular}{|c|} 
Drum \\
Activity \\
(nCi)
\end{tabular} & $\begin{array}{l}C_{f} \text { of } \\
\ln 1 / T\end{array}$ & $\begin{array}{l}\text { Corr. } \\
\text { Sum } \\
\text { (cps) }\end{array}$ & $\begin{array}{c}\text { Drum } \\
\text { Activity } \\
\text { (dps) }\end{array}$ & \begin{tabular}{|c|} 
Drum \\
Activity \\
(nCi)
\end{tabular} \\
\hline & T3567D1 & 300 & 31 & 32 & 9.92 & 0.103 & 0.03 & & & & & & & & & & \\
\hline & T3567D2 & 300 & 521 & 4.99 & 26 & 1.737 & 0.09 & & & & & & & & & & \\
\hline & T3567D3 & 300 & 572 & 4.58 & 26.2 & 1.907 & \begin{tabular}{|l|}
0.09 \\
\end{tabular} & 0.90 & 0.951 & & & & & 0.961 & & & \\
\hline & & & & & & & & & & & & & & & & & \\
\hline & 567D1 & 600 & 75 & 15.6 & 11.7 & 0.125 & \begin{tabular}{|l|}
0.02 \\
\end{tabular} & & & 0.16 & & & & 0.1486 & & & \\
\hline & 567D2 & 600 & 98 & 13.6 & 13.33 & 0.163 & 0.02 & & & 1.12 & 1.338 & 2852.9 & 77.029 & 0.5188 & 0.73 & 1556.3 & 42.021 \\
\hline & 567D3 & 600 & 39 & 27.21 & 10.61 & 0.065 & 0.02 & & & 0.06 & & & & 0.0625 & & & \\
\hline & & & & & & & & & & & & & & & & & \\
\hline \multirow[t]{15}{*}{ FD416 } & T1416D1 & 300 & 3346 & 1.79 & 59.89 & 11.15 & 0.2 & 1.52 & 1.234 & & & & & 1.174 & & & \\
\hline & T1416D2 & 300 & 351 & 6.32 & 22.18 & 1.17 & 0.07 & & & & & & & & & & \\
\hline & T1416D3 & 300 & 1001 & 3.43 & 34.33 & 3.337 & 0.11 & & & & & & & & & & \\
\hline & & & & & & & & & & & & & & & & & \\
\hline & T2416D1 & 166.01 & 203 & 8.16 & 16.56 & 1.223 & 0.1 & & & & & & & & & & \\
\hline & T2416D2 & 166.01 & 1137 & 3.15 & 35.82 & 6.849 & 0.22 & 2.60 & 1.612 & & & & & 1.4213 & & & \\
\hline & T2416D3 & 166.01 & 127 & 5.33 & 6.769 & 0.765 & 0.04 & & & & & & & & & & \\
\hline & & & & & & & & & & & & & & & & & \\
\hline & T3416D1 & 300 & 752 & 3.94 & 29.63 & 2.507 & 0.1 & & & & & & & & & & \\
\hline & \begin{tabular}{|l|} 
T3416D2 \\
\end{tabular} & 300 & 1185 & 3.09 & 36.62 & 3.95 & 0.12 & & & & & & & & & & \\
\hline & T3416D3 & 300 & 351 & 6.28 & 22.04 & 1.17 & 0.07 & 23.48 & 4.845 & & & & & 2.7045 & & & \\
\hline & & & & & & & & & & & & & & & & & \\
\hline & 416D1 & 2000 & 919 & 3.7 & 34 & 0.46 & 0.02 & & & 0.57 & & & & 0.5395 & & & \\
\hline & 416D2 & 2000 & 1169 & 3.38 & 39.51 & 0.585 & 0.02 & & & 0.94 & 3.818 & 8140.5 & 219.79 & 0.8307 & 2.659 & 5669.2 & 153.07 \\
\hline & 416D3 & 2000 & 953 & 3.78 & 36.02 & 0.477 & \begin{tabular}{|l|}
0.02 \\
\end{tabular} & & & 2.31 & & & & 1.2887 & & & \\
\hline & & & & & & & & & & & & & & & & & \\
\hline \multirow[t]{5}{*}{ FD580 } & T1580 & 300 & 3659 & 1.72 & 62.93 & 12.2 & 0.21 & 1.34 & 1.157 & & & & & 1.1188 & & & \\
\hline & T1580 & 300 & 2397 & 2.14 & 51.3 & 7.99 & \begin{tabular}{|l|} 
\\
\end{tabular} & & & & & & & & & & \\
\hline & T1580 & 300 & 790 & 3.87 & 30.57 & 2.633 & 0.1 & & & & & & & & & & \\
\hline & & & & & & & & & & & & & & & & & \\
\hline & & & & & & & & & & & & & & & & & \\
\hline
\end{tabular}


WSRC-TR-2001-00004

Revision 0

January 8, 2001

Page 34 of 69

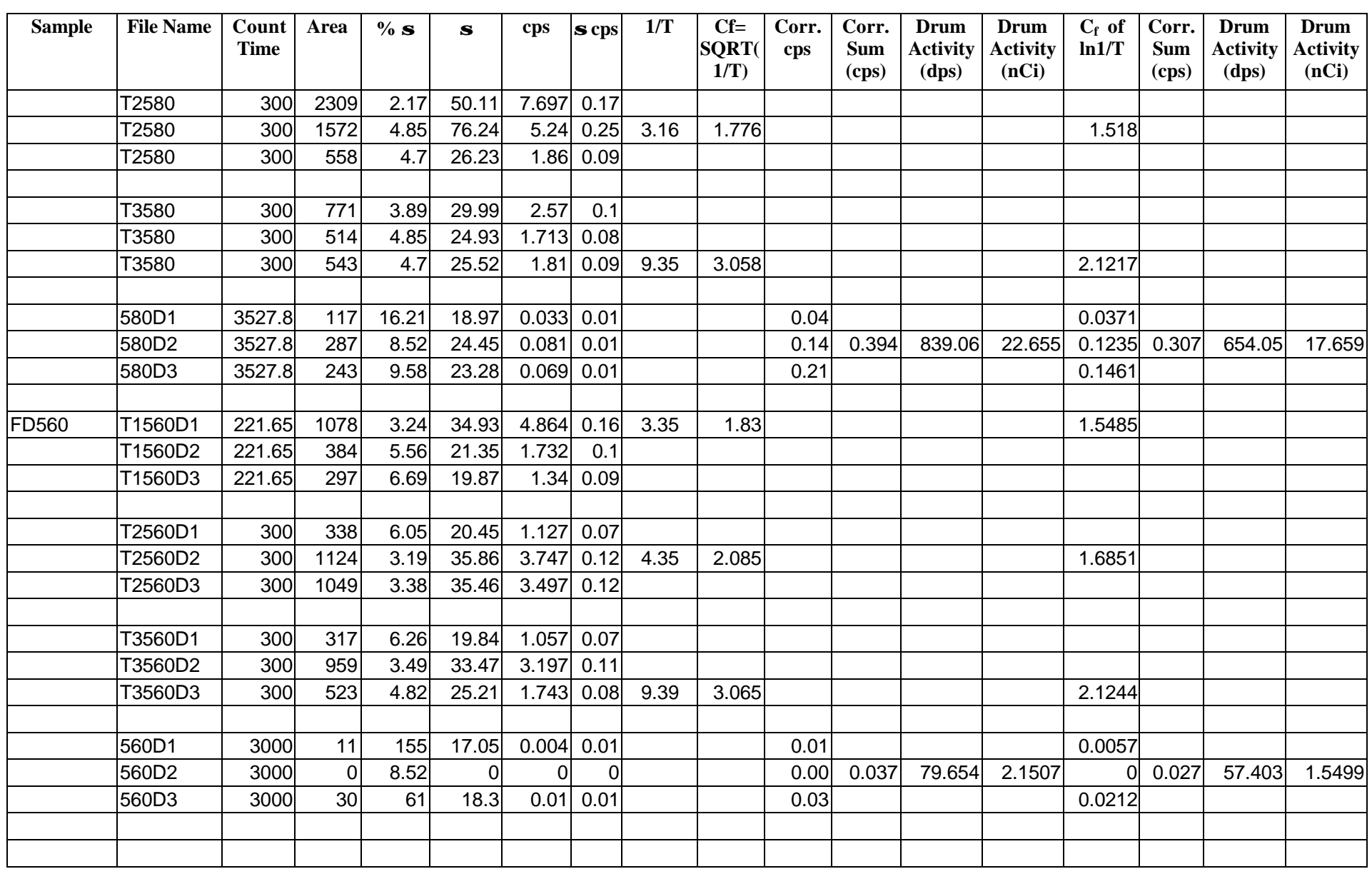


WSRC-TR-2001-00004

Revision 0

January 8, 2001

Page 35 of 69

\begin{tabular}{|c|c|c|c|c|c|c|c|c|c|c|c|c|c|c|c|c|c|}
\hline Sample & \begin{tabular}{|l|} 
File Name \\
\end{tabular} & $\begin{array}{l}\text { Count } \\
\text { Time }\end{array}$ & Area & $\% \sigma$ & $\sigma$ & cps & $\sigma$ cps & $1 / T$ & \begin{tabular}{|c|} 
Cf $=$ \\
SQRT( \\
1/T)
\end{tabular} & $\begin{array}{c}\text { Corr. } \\
\text { cps }\end{array}$ & $\begin{array}{l}\text { Corr. } \\
\text { Sum } \\
\text { (cps) }\end{array}$ & $\begin{array}{c}\text { Drum } \\
\text { Activity } \\
\text { (dps) }\end{array}$ & \begin{tabular}{|c|} 
Drum \\
Activity \\
(nCi)
\end{tabular} & $\begin{array}{l}C_{f} \text { of } \\
\ln 1 / T\end{array}$ & $\begin{array}{l}\text { Corr. } \\
\text { Sum } \\
\text { (cps) }\end{array}$ & $\begin{array}{c}\text { Drum } \\
\text { Activity } \\
\text { (dps) }\end{array}$ & $\begin{array}{c}\text { Drum } \\
\text { Activity } \\
\text { (nCi) }\end{array}$ \\
\hline \multirow[t]{15}{*}{\begin{tabular}{|l|} 
FD558 \\
\end{tabular}} & T1558D1 & 300 & 2564 & 2.07 & 53.07 & 8.547 & \begin{tabular}{l|l}
0.18 \\
\end{tabular} & 1.91 & 1.382 & & & & & 1.2755 & & & \\
\hline & T1558D2 & 300 & 2403 & 2.17 & 52.15 & 8.01 & 0.17 & & & & & & & & & & \\
\hline & T1558D3 & 300 & 515 & 3.94 & 20.29 & 1.717 & 0.07 & & & & & & & & & & \\
\hline & & & & & & & & & & & & & & & & & \\
\hline & T2558D1 & 300 & 2078 & 2.3 & 47.79 & 6.927 & 0.16 & & & & & & & & & & \\
\hline & T2558D2 & 300 & 1425 & 2.85 & 40.61 & 4.75 & 0.14 & 3.47 & 1.863 & & & & & 1.5666 & & & \\
\hline & T2558D3 & 300 & 608 & 4.43 & 26.93 & 2.027 & 0.09 & & & & & & & & & & \\
\hline & & & & & & & & & & & & & & & & & \\
\hline & T3558D1 & 300 & 749 & 3.96 & 29.66 & 2.497 & 0.1 & & & & & & & & & & \\
\hline & T3558D2 & 300 & 434 & 5.56 & 24.13 & 1.447 & 0.08 & & & & & & & & & & \\
\hline & T3558D3 & 300 & 689 & 4.13 & 28.46 & 2.297 & 0.09 & 7.25 & 2.693 & & & & & 1.9714 & & & \\
\hline & & & & & & & & & & & & & & & & & \\
\hline & 558D1 & 2211.7 & 55 & 30.68 & $\begin{array}{l}16.87 \\
\end{array}$ & 0.025 & 0.01 & & & & & & & & & & \\
\hline & 558D2 & 2211.7 & 130 & 16.48 & 21.42 & 0.059 & 0.01 & & & & & & & & & & \\
\hline & 558D3 & 2211.7 & 113 & 17.82 & 20.14 & 0.051 & 0.01 & & & & & & & & & & \\
\hline
\end{tabular}


WSRC-TR-2001-00004 Revision 0

January 8, 2001

Page 36 of 69

\begin{tabular}{|c|c|c|c|c|c|c|c|c|c|c|c|c|c|c|c|c|c|}
\hline Sample & File Name & \begin{tabular}{|l} 
Count \\
Time
\end{tabular} & Area & $\% \sigma$ & $\sigma$ & cps & $\sigma$ cps & $1 / T$ & \begin{tabular}{|c|}
$\mathbf{C f}=$ \\
SQRT( \\
1/T)
\end{tabular} & $\begin{array}{c}\text { Corr. } \\
\text { cps }\end{array}$ & $\begin{array}{l}\text { Corr. } \\
\text { Sum } \\
\text { (cps) }\end{array}$ & \begin{tabular}{|c|} 
Drum \\
Activity \\
(dps)
\end{tabular} & $\begin{array}{c}\text { Drum } \\
\text { Activity } \\
\text { (nCi) }\end{array}$ & $\begin{array}{l}C_{f} \text { of } \\
\ln 1 / T\end{array}$ & $\begin{array}{l}\text { Corr. } \\
\text { Sum } \\
\text { (cps) }\end{array}$ & $\begin{array}{c}\text { Drum } \\
\text { Activity } \\
\text { (dps) }\end{array}$ & $\begin{array}{c}\text { Drum } \\
\text { Activity } \\
\text { (nCi) }\end{array}$ \\
\hline$T_{01}$ & T01det1 & 100 & 1673 & 2.51 & 41.99 & 16.73 & 0.42 & & & & & & & & & & \\
\hline$T_{01}$ & T01det3 & 100 & 1199 & 2.97 & 41.99 & 11.99 & 0.42 & & & & & & & & & & \\
\hline & & & & & & & & & & & & & & & & & \\
\hline $\mathrm{T}_{02}$ & T02det1 & 69.06 & 990 & 3.29 & 32.57 & 14.34 & 0.47 & & & & & & & & & & \\
\hline $\mathrm{T}_{02}$ & T02det3 & \begin{tabular}{|l|}
69.06 \\
\end{tabular} & 1046 & 3.21 & 32.57 & 15.15 & \begin{tabular}{|l|}
0.47 \\
\end{tabular} & & & & & & & & & & \\
\hline & & & & & & & & & & & & & & & & & \\
\hline $\mathrm{T}_{03}$ & \begin{tabular}{|l|} 
T03det1 \\
\end{tabular} & \begin{tabular}{|l|}
72.82 \\
\end{tabular} & 807 & 3.66 & 29.54 & 11.08 & 0.41 & & & & & & & & & & \\
\hline $\mathrm{T}_{03}$ & T03det2 & \begin{tabular}{|l|}
72.82 \\
\end{tabular} & 1163 & 3 & 34.89 & 15.97 & 0.48 & & & & & & & & & & \\
\hline$T_{03}$ & T03det3 & \begin{tabular}{|l|}
72.82 \\
\end{tabular} & 1098 & 3.13 & 29.54 & 15.08 & 0.41 & & & & & & & & & & \\
\hline & & & & & & & & & & & & & & & & & \\
\hline & \begin{tabular}{|l|} 
T2418D1 \\
\end{tabular} & 600 & 3510 & 1.8 & 63.18 & 5.85 & 0.11 & & & & & & & & & & \\
\hline & T2418D2 & 600 & 1376 & 3 & 41.28 & 2.29 & 0.07 & 12.65 & 3.557 & & & & & 2.31 & & & \\
\hline & \begin{tabular}{|l|} 
T2418D3 \\
\end{tabular} & 600 & 1704 & 2.7 & 46.01 & 2.84 & 0.08 & & & & & & & & & & \\
\hline & & & & & & & & & & & & & & & & & \\
\hline & T3418D1 & 208.06 & 1644 & 2.66 & 43.73 & 7.90 & 0.21 & & & & & & & & & & \\
\hline & \begin{tabular}{|l|} 
T3418D2 \\
\end{tabular} & 208.06 & 1381 & 2.97 & 41.02 & 6.64 & 0.20 & & & & & & & & & & \\
\hline & T3418D3 & 208.06 & 3325 & 1.28 & 42.56 & 15.98 & 0.20 & 1.14 & 1.069 & & & & & 1.05 & & & \\
\hline & & & & & & & & & & & & & & & & & \\
\hline & & & & & & & & & & & & & & & & & \\
\hline & & & & & & & & & & & & & & & & & \\
\hline
\end{tabular}


WSRC-TR-2001-00004

Revision 0

January 8, 200

Page 37 of 69

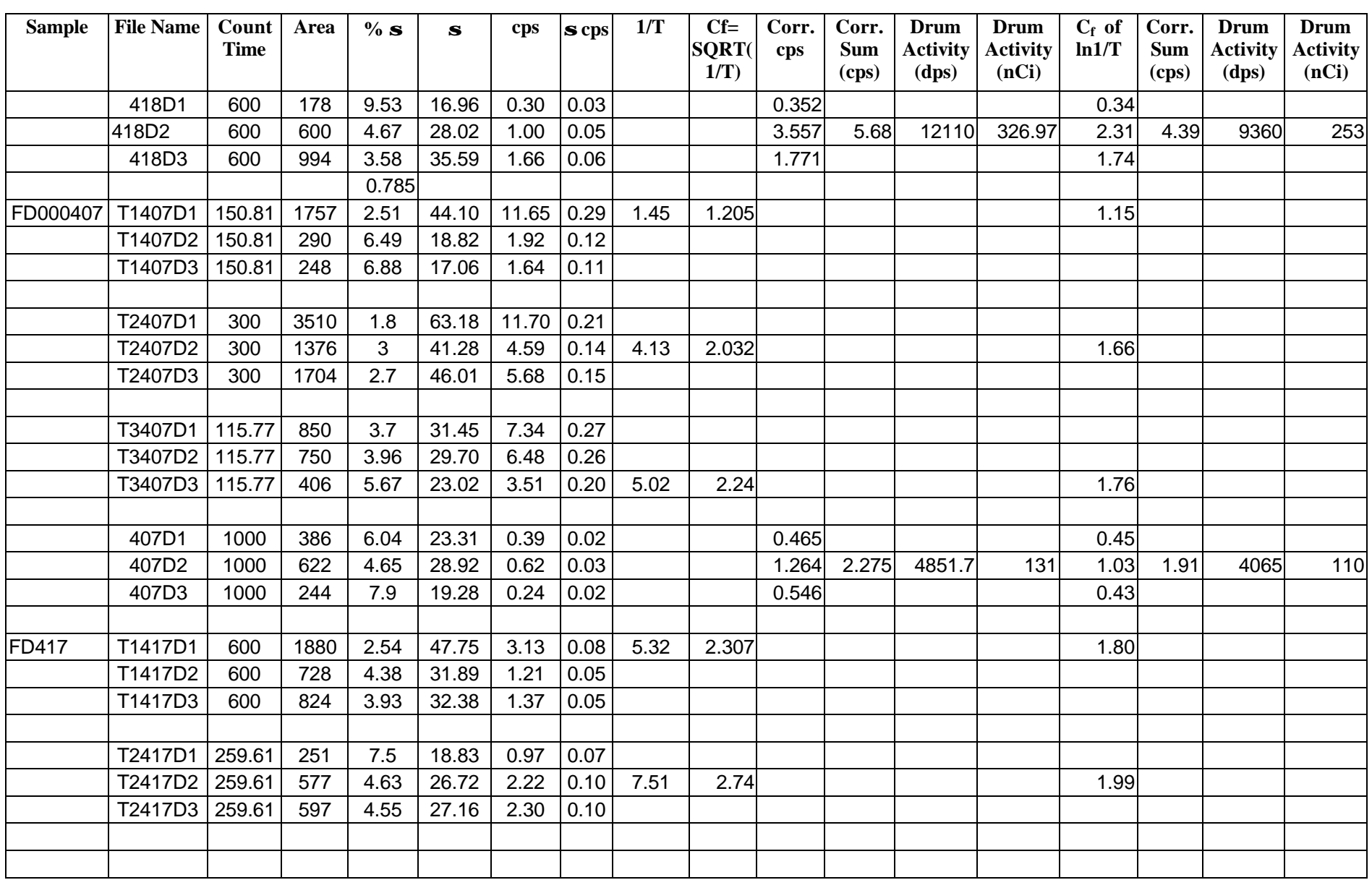


WSRC-TR-2001-00004

Revision 0

January 8, 200

Page 38 of 69

\begin{tabular}{|c|c|c|c|c|c|c|c|c|c|c|c|c|c|c|c|c|c|}
\hline Sample & File Name & \begin{tabular}{|l|} 
Count \\
Time
\end{tabular} & Area & $\% \sigma$ & $\sigma$ & cps & $\sigma \mathrm{cps}$ & $1 / T$ & \begin{tabular}{|c|} 
Cf $=$ \\
SQRT( \\
1/T)
\end{tabular} & $\begin{array}{c}\text { Corr. } \\
\text { cps }\end{array}$ & $\begin{array}{l}\text { Corr. } \\
\text { Sum } \\
\text { (cps) }\end{array}$ & $\begin{array}{c}\text { Drum } \\
\text { Activity } \\
\text { (dps) }\end{array}$ & \begin{tabular}{|c|} 
Drum \\
Activity \\
(nCi)
\end{tabular} & $\begin{array}{l}C_{\mathrm{f}} \text { of } \\
\ln 1 / T\end{array}$ & $\begin{array}{l}\text { Corr. } \\
\text { Sum } \\
\text { (cps) }\end{array}$ & $\begin{array}{c}\text { Drum } \\
\text { Activity } \\
\text { (dps) }\end{array}$ & $\begin{array}{c}\text { Drum } \\
\text { Activity } \\
\text { (nCi) }\end{array}$ \\
\hline & T3417D1 & \begin{tabular}{|l|}
445.64 \\
\end{tabular} & 490 & 5.21 & 25.53 & 1.10 & \begin{tabular}{|l|}
0.06 \\
\end{tabular} & & & & & & & & & & \\
\hline & T3417D3 & 445.64 & 3547 & 1.77 & 62.78 & 7.96 & 0.14 & 2.07 & 1.437 & & & & & 1.31 & & & \\
\hline & & & & & & & & & & & & & & & & & \\
\hline & 417D1 & 1000 & 59 & 22.39 & 13.21 & 0.06 & 0.01 & & & 0.136 & & & & 0.11 & & & \\
\hline \multicolumn{18}{|c|}{ 9/27 Acquisitions } \\
\hline$T_{01}$ & T01det1 & 100 & 1610 & 2.57 & 41.38 & 16.10 & 0.41 & & & & & & & & & & \\
\hline $\mathrm{T}_{01}$ & T01det2 & 100 & 1701 & 2.5 & 42.53 & 17.01 & 0.43 & & & & & & & & & & \\
\hline$T_{01}$ & T01det3 & 100 & 1214 & 2.98 & 41.38 & 12.14 & 0.41 & & & & & & & & & & \\
\hline & & & & & & & & & & & & & & & & & \\
\hline $\mathrm{T}_{02}$ & T02det1 & 100 & 1314 & 2.89 & 37.97 & 13.14 & 0.38 & & & & & & & & & & \\
\hline $\mathrm{T}_{03}$ & T03det1 & 72.82 & 807 & 3.66 & 29.54 & 11.08 & 0.41 & & & & & & & & & & \\
\hline $\mathrm{T}_{03}$ & T03det2 & 72.82 & 1163 & 3 & 34.89 & 15.97 & 0.48 & & & & & & & & & & \\
\hline \multirow[t]{2}{*}{$\mathrm{T}_{03}$} & T03det3 & 55 & 850 & 3.73 & 29.54 & 15.45 & 0.54 & & & & & & & & & & \\
\hline & & & & & & & & 16.13 & & & & & & & & & \\
\hline \multirow[t]{7}{*}{ FD385 } & T1385D1 & 84.35 & 977 & 3.41 & 33.32 & 11.58 & 0.39 & 1.43 & 1.195 & & & & & 1.15 & & & \\
\hline & T1385D2 & 84.35 & 921 & 3.45 & 31.77 & 10.92 & 0.38 & & & & & & & & & & \\
\hline & T1385D3 & 84.35 & 179 & 7.94 & 14.21 & 2.12 & 0.17 & & & & & & & & & & \\
\hline & & & & & & & & & & & & & & & & & \\
\hline & T2385D1 & 300 & 2686 & 2.04 & 54.79 & 8.95 & 0.18 & & & & & & & & & & \\
\hline & T2385D2 & 300 & 1592 & 2.73 & 43.46 & 5.31 & 0.14 & 3.60 & 1.898 & & & & & 1.59 & & & \\
\hline & T2385D3 & 300 & 235 & 7.37 & 17.32 & 0.78 & 0.06 & & & & & & & & & & \\
\hline
\end{tabular}


WSRC-TR-2001-00004

Revision 0

January 8, 200

Page 39 of 69

\begin{tabular}{|c|c|c|c|c|c|c|c|c|c|c|c|c|c|c|c|c|c|}
\hline Sample & File Name & \begin{tabular}{|l|} 
Count \\
Time
\end{tabular} & Area & $\% \sigma$ & $\sigma$ & cps & $\sigma$ cps & $1 / T$ & $\begin{array}{c}\text { Cf }= \\
\text { SQRT( }( \\
\text { 1/T) }\end{array}$ & $\begin{array}{c}\text { Corr. } \\
\text { cps }\end{array}$ & $\begin{array}{l}\text { Corr. } \\
\text { Sum } \\
\text { (cps) }\end{array}$ & \begin{tabular}{|c|} 
Drum \\
Activity \\
(dps)
\end{tabular} & $\begin{array}{c}\text { Drum } \\
\text { Activity } \\
\text { (nCi) }\end{array}$ & $\begin{array}{l}C_{\mathrm{f}} \text { of } \\
\ln 1 / \mathrm{T}\end{array}$ & \begin{tabular}{|l|} 
Corr. \\
Sum \\
(cps)
\end{tabular} & \begin{tabular}{|c|} 
Drum \\
Activity \\
(dps)
\end{tabular} & $\begin{array}{c}\text { Drum } \\
\text { Activity } \\
\text { (nCi) }\end{array}$ \\
\hline & T3385D1 & 120.99 & 371 & 5.67 & 21.04 & 3.07 & 0.17 & & & & & & & & & & \\
\hline & T3385D3 & 120.99 & 62 & 16.01 & 9.93 & 0.51 & 0.08 & -249.86 & $\mathrm{~N} / \mathrm{A}$ & & & & & $\mathrm{N} / \mathrm{A}$ & & & \\
\hline & & & & & & & & & & & & & & & & & \\
\hline & 385D1 & 5000 & 1458 & 3.17 & 46.22 & 0.29 & 0.01 & & & 0.349 & & & & 0.33 & & & \\
\hline & & & & & & & & & & & & & & & & & \\
\hline \multirow[t]{11}{*}{\begin{tabular}{|l|} 
FD378 \\
\end{tabular}} & T1378D1 & 289.35 & 7990 & 1.19 & 95.08 & 27.61 & 0.33 & 1.35 & 1.16 & & & & & 1.12 & & & \\
\hline & T1378D2 & 289.35 & 13087 & 0.93 & 121.71 & 45.23 & 0.42 & & & & & & & & & & \\
\hline & T1378D3 & 289.35 & 6215 & 1.37 & 85.15 & 21.48 & 0.29 & & & & & & & & & & \\
\hline & & & & & & & & & & & & & & & & & \\
\hline & T2378D1 & 600 & 15568 & 0.86 & 133.88 & 25.95 & 0.22 & & & & & & & & & & \\
\hline & T3378D2 & 300 & 11629 & 0.99 & 115.13 & 38.76 & 0.38 & & & & & & & & & & \\
\hline & T3378D3 & 300 & \begin{tabular}{|l|}
4585 \\
\end{tabular} & 1.62 & \begin{tabular}{|l|}
74.28 \\
\end{tabular} & 15.28 & 0.25 & 36.02 & 6.002 & & & & & 2.99 & & & \\
\hline & & & & & & & & & & & & & & & & & \\
\hline & 378D1 & 1130 & 17658 & 0.82 & 144.80 & 15.63 & 0.13 & & & 18.13 & & & & 17.52 & & & \\
\hline & 378D2 & 1130 & 37703 & 0.55 & 207.37 & 33.37 & 0.18 & & & 38.31 & 145.5 & \begin{tabular}{|l|}
310189 \\
\end{tabular} & 8375.1 & 37.11 & 99.04 & \begin{tabular}{|l|}
211168 \\
\end{tabular} & 5702 \\
\hline & 378D3 & 1130 & $16764 \mid$ & 0.84 & 140.82 & 14.84 & 0.12 & & & 89.04 & & & & 44.40 & & & \\
\hline & & & & & & & & & & & & & & & & & \\
\hline \multirow[t]{5}{*}{\begin{tabular}{|l|} 
FD394 \\
\end{tabular}} & T1394D1 & 300 & 4785 & 1.56 & 74.65 & 15.95 & 0.25 & 1.47 & 1.214 & & & & & 1.16 & & & \\
\hline & T1394D2 & 300 & 6276 & 1.38 & \begin{tabular}{|l|}
86.61 \\
\end{tabular} & 20.92 & 0.29 & & & & & & & & & & \\
\hline & T1394D3 & 300 & 5112 & 1.56 & \begin{tabular}{|l|}
79.75 \\
\end{tabular} & 17.04 & 0.27 & & & & & & & & & & \\
\hline & & & & & & & & & & & & & & & & & \\
\hline & & & & & & & & & & & & & & & & & \\
\hline
\end{tabular}


WSRC-TR-2001-00004

Revision 0

January 8, 200

Page 40 of 69

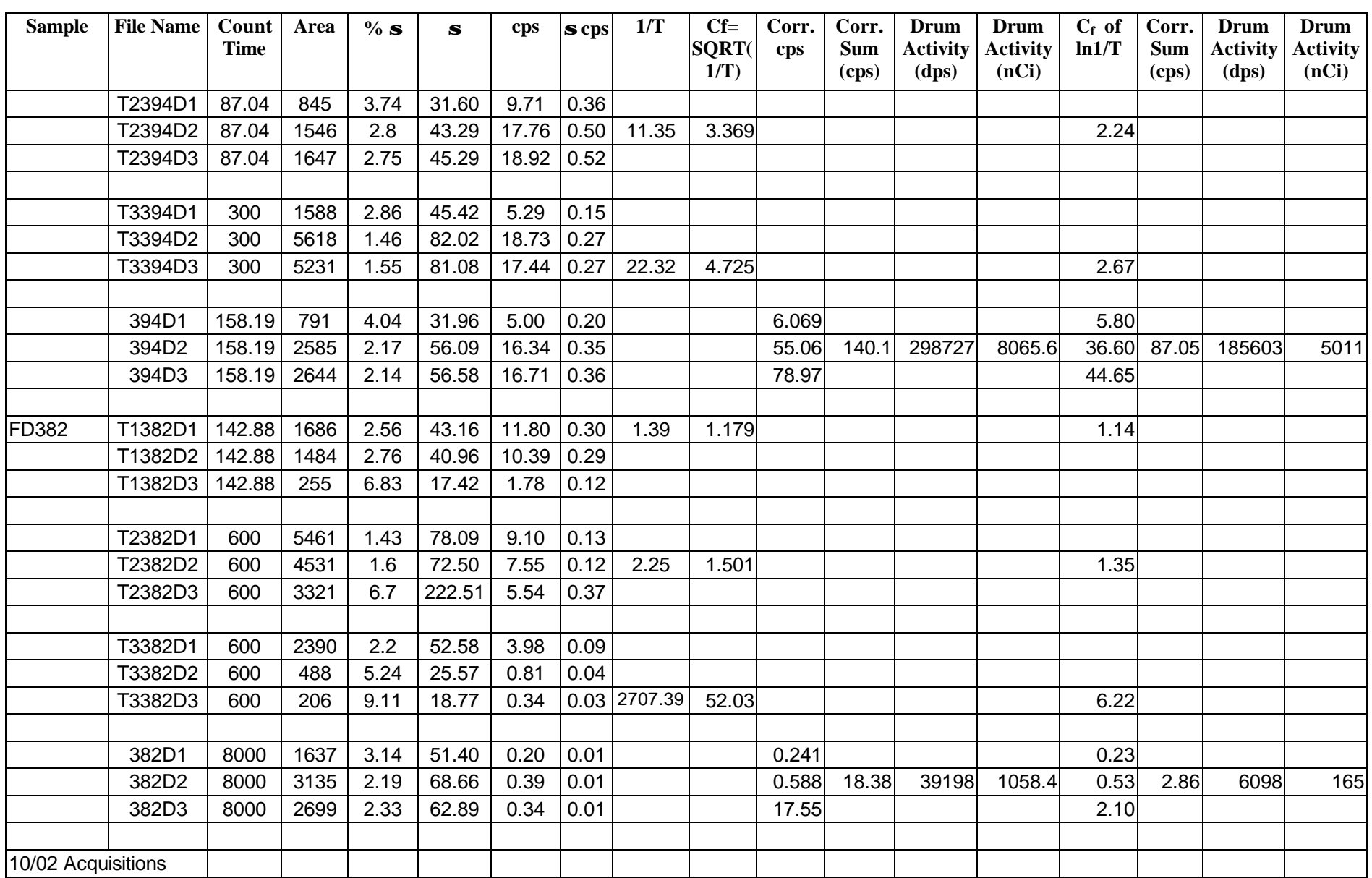


WSRC-TR-2001-00004

Revision 0

January 8, 200

Page 41 of 69

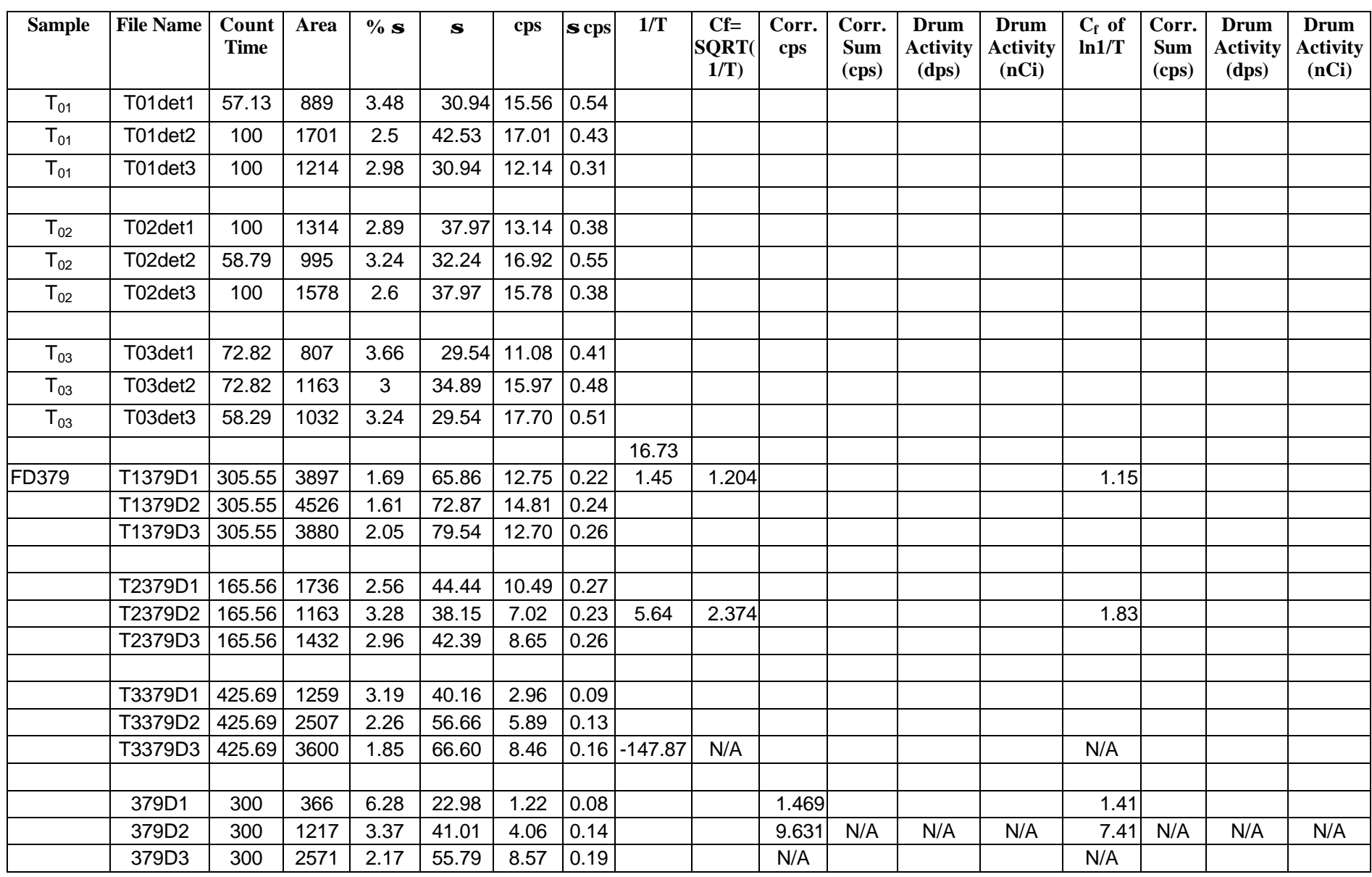


WSRC-TR-2001-00004

Revision 0

January 8, 200

Page 42 of 69

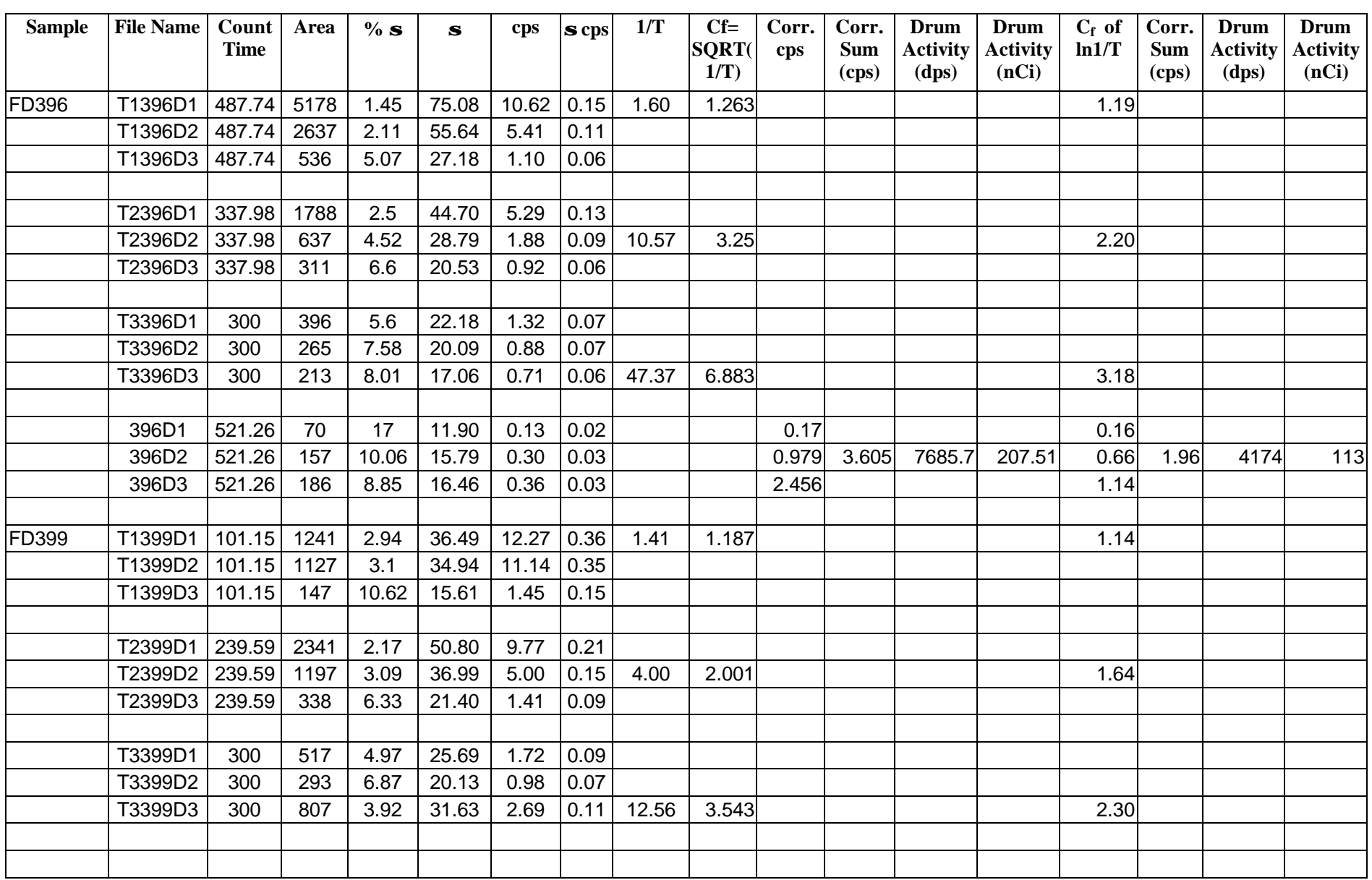


WSRC-TR-2001-00004

Revision 0

January 8, 200

Page 43 of 69

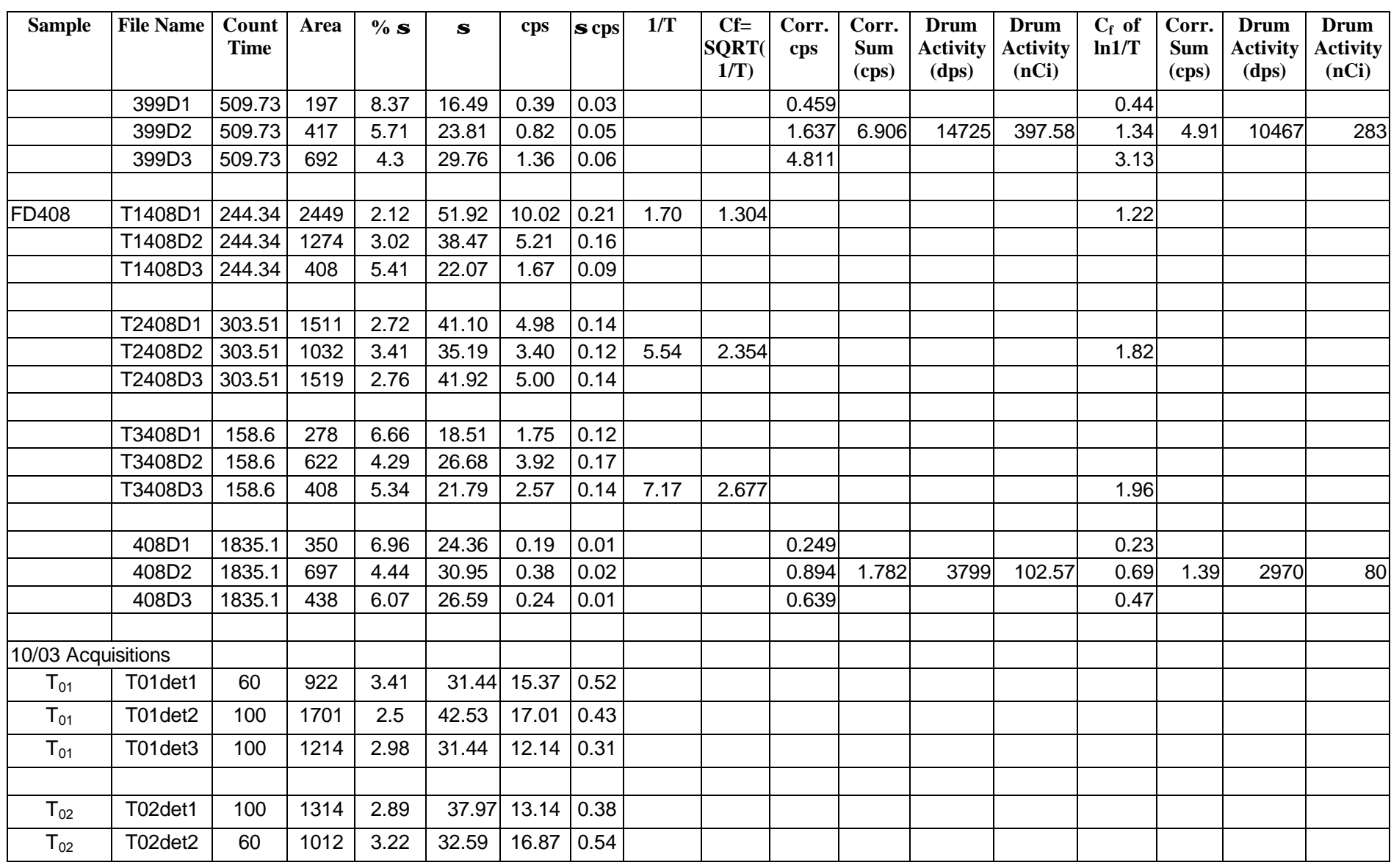


WSRC-TR-2001-00004

Revision 0

January 8, 200

Page 44 of 69

\begin{tabular}{|c|c|c|c|c|c|c|c|c|c|c|c|c|c|c|c|c|c|}
\hline$T_{02}$ & T02det3 & 100 & 1578 & 2.6 & 37.97 & 15.78 & 0.38 & & & & & & & & & & \\
\hline Sample & File Name & $\begin{array}{c}\text { Count } \\
\text { Time }\end{array}$ & Area & $\% \sigma$ & $\sigma$ & cps & $\sigma$ cps & $1 / \mathrm{T}$ & \begin{tabular}{|c|} 
Cf $=$ \\
SQRT( \\
1/T)
\end{tabular} & $\begin{array}{c}\text { Corr. } \\
\text { cps }\end{array}$ & $\begin{array}{l}\text { Corr. } \\
\text { Sum } \\
\text { (cps) }\end{array}$ & \begin{tabular}{|c|} 
Drum \\
Activity \\
(dps)
\end{tabular} & $\begin{array}{c}\text { Drum } \\
\text { Activity } \\
\text { (nCi) }\end{array}$ & $\begin{array}{l}C_{f} \text { of } \\
\ln 1 / T\end{array}$ & $\begin{array}{l}\text { Corr. } \\
\text { Sum } \\
\text { (cps) }\end{array}$ & \begin{tabular}{|c|} 
Drum \\
Activity \\
(dps)
\end{tabular} & \begin{tabular}{|c|} 
Drum \\
Activity \\
(nCi)
\end{tabular} \\
\hline$T_{03}$ & T03det2 & 72.82 & 1163 & 3 & 34.89 & 15.97 & 0.48 & & & & & & & & & & \\
\hline \multirow[t]{2}{*}{$\mathrm{T}_{03}$} & T03det3 & 60 & 995 & 3.27 & 29.54 & 16.58 & 0.49 & & & & & & & & & & \\
\hline & & & & & & & & 16.27 & & & & & & & & & \\
\hline \multirow{10}{*}{ FD403 } & \begin{tabular}{|l|} 
T1403D2 \\
\end{tabular} & 233.24 & 2188 & 2.27 & 49.67 & 9.38 & 0.21 & & & & & & & & & & \\
\hline & T1403D3 & 233.24 & 1491 & 2.77 & 41.30 & 6.39 & 0.18 & & & & & & & & & & \\
\hline & & & & & & & & & & & & & & & & & \\
\hline & T2403D1 & 206.13 & 1384 & 2.84 & 39.31 & 6.71 & 0.19 & & & & & & & & & & \\
\hline & T2403D2 & 206.13 & 1665 & 2.63 & 43.79 & 8.08 & 0.21 & 2.30 & 1.518 & & & & & 1.36 & & & \\
\hline & T2403D3 & 206.13 & 1705 & 2.6 & 44.33 & 8.27 & 0.22 & & & & & & & & & & \\
\hline & & & & & & & & & & & & & & & & & \\
\hline & 403D1 & 600 & 229 & 7.74 & 17.72 & 0.38 & 0.03 & & & 0.517 & & & & 0.48 & & & \\
\hline & 403D2 & 600 & 608 & 4.35 & 26.45 & 1.01 & \begin{tabular}{|l|}
0.04 \\
\end{tabular} & & & 1.538 & 7.313 & 15594 & 421.03 & 1.38 & 5.76 & 12280 & 332 \\
\hline & 403D3 & 600 & 1208 & 3.11 & 37.57 & 2.01 & 0.06 & & & 5.258 & & & & 3.90 & & & \\
\hline & & & & & & & & & & & & & & & & & \\
\hline \multirow[t]{7}{*}{ FD556 } & T1556D1 & 136.94 & 1625 & 2.63 & 42.74 & 11.87 & 0.31 & 1.42 & 1.191 & & & & & 1.14 & & & \\
\hline & \begin{tabular}{|l|} 
T1556D2 \\
\end{tabular} & 136.94 & 491 & 4.83 & 23.72 & 3.59 & 0.17 & & & & & & & & & & \\
\hline & T1556D3 & 136.94 & 414 & 5.44 & 22.52 & 3.02 & 0.16 & & & & & & & & & & \\
\hline & & & & & & & & & & & & & & & & & \\
\hline & T2556D1 & 375.64 & 699 & 4.39 & 30.69 & 1.86 & 0.08 & & & & & & & & & & \\
\hline & T2556D2 & 375.64 & 2880 & 1.99 & 57.31 & 7.67 & 0.15 & 2.35 & 1.533 & & & & & 1.37 & & & \\
\hline & T2556D3 & 375.64 & 1693 & 2.65 & 44.86 & 4.51 & 0.12 & & & & & & & & & & \\
\hline
\end{tabular}


WSRC-TR-2001-00004

Revision 0

January 8, 2001

Page 45 of 69

\begin{tabular}{|c|c|c|c|c|c|c|c|c|c|c|c|c|c|c|c|c|c|}
\hline & & & & & & & & & & & & & & & & & \\
\hline \multirow[t]{7}{*}{ Sample } & File Name & \begin{tabular}{|l|} 
Count \\
Time
\end{tabular} & Area & $\% \sigma$ & $\sigma$ & cps & $\sigma$ cps & $1 / T$ & \begin{tabular}{|c|} 
Cf $=$ \\
SQRT( ( \\
1/T)
\end{tabular} & $\begin{array}{c}\text { Corr. } \\
\text { cps }\end{array}$ & $\begin{array}{l}\text { Corr. } \\
\text { Sum } \\
\text { (cps) }\end{array}$ & $\begin{array}{c}\text { Drum } \\
\text { Activity } \\
\text { (dps) }\end{array}$ & \begin{tabular}{|c|} 
Drum \\
Activity \\
(nCi)
\end{tabular} & $\begin{array}{l}C_{f} \text { of } \\
\ln 1 / T\end{array}$ & $\begin{array}{l}\text { Corr. } \\
\text { Sum } \\
\text { (cps) }\end{array}$ & $\begin{array}{c}\text { Drum } \\
\text { Activity } \\
\text { (dps) }\end{array}$ & $\begin{array}{c}\text { Drum } \\
\text { Activity } \\
\text { (nCi) }\end{array}$ \\
\hline & T3556D1 & 210.06 & 396 & 4.39 & 17.38 & 1.89 & 0.08 & & & & & & & & & & \\
\hline & T3556D2 & 210.06 & 716 & 4.21 & 30.14 & 3.41 & 0.14 & & & & & & & & & & \\
\hline & T3556D3 & 210.06 & 918 & 3.56 & 32.68 & 4.37 & \begin{tabular}{|l|}
0.16 \\
\end{tabular} & 4.23 & 2.055 & & & & & 1.67 & & & \\
\hline & 556D2 & 597.56 & 443 & 6.08 & 26.93 & 0.74 & 0.05 & & & 1.136 & 2.677 & 5708.2 & 154.12 & 1.02 & 2.34 & 4988 & 135 \\
\hline & 556D3 & 597.56 & 310 & 6.9 & 21.39 & 0.52 & 0.04 & & & 1.066 & & & & 0.87 & & & \\
\hline & & & & & & & & & & & & & & & & & \\
\hline \multirow[t]{11}{*}{\begin{tabular}{|l|} 
FD557 \\
\end{tabular}} & T1557D1 & \begin{tabular}{|l|}
304.74 \\
\end{tabular} & 1778 & 2.58 & 45.87 & 5.83 & \begin{tabular}{|l|}
0.15 \\
\end{tabular} & 3.13 & 1.768 & & & & & 1.51 & & & \\
\hline & T1557D2 & 304.74 & 1579 & 2.74 & 43.26 & 5.18 & 0.14 & & & & & & & & & & \\
\hline & T1557D3 & 304.74 & 997 & 3.48 & 34.70 & 3.27 & 0.11 & & & & & & & & & & \\
\hline & & & & & & & & & & & & & & & & & \\
\hline & T3557D1 & 300 & 713 & 4.24 & 30.23 & 2.38 & 0.10 & & & & & & & & & & \\
\hline & T3557D2 & 300 & 1886 & 2.51 & 47.34 & 6.29 & 0.16 & & & & & & & & & & \\
\hline & T3557D3 & 300 & 3239 & 1.85 & 59.92 & 10.80 & 0.20 & 1.70 & 1.304 & & & & & 1.22 & & & \\
\hline & & & & & & & & & & & & & & & & & \\
\hline & 557D1 & 600 & 378 & 6.03 & 22.79 & 0.63 & \begin{tabular}{|l|l|}
0.04 \\
\end{tabular} & & & 1.114 & & & & 0.95 & & & \\
\hline & 557D2 & 600 & 672 & 4.52 & 30.37 & 1.12 & 0.05 & & & 2.212 & 4.921 & 10492 & 283.29 & 1.82 & 4.27 & 9109 & 246 \\
\hline & 557D3 & 600 & 734 & 4.13 & 30.31 & 1.22 & 0.05 & & & 1.595 & & & & 1.50 & & & \\
\hline & & & & & & & & & & & & & & & & & \\
\hline \multirow[t]{3}{*}{\begin{tabular}{|l|} 
FD573 \\
\end{tabular}} & T1573D1 & \begin{tabular}{|l|}
140.99 \\
\end{tabular} & 1827 & 2.43 & 44.40 & 12.96 & \begin{tabular}{|l|}
0.31 \\
\end{tabular} & 1.27 & 1.127 & & & & & 1.10 & & & \\
\hline & T1573D2 & 140.99 & 1366 & 2.86 & 39.07 & 9.69 & 0.28 & & & & & & & & & & \\
\hline & T1573D3 & 140.99 & 368 & 5.95 & 21.90 & 2.61 & \begin{tabular}{|l|}
0.16 \\
\end{tabular} & & & & & & & & & & \\
\hline
\end{tabular}


WSRC-TR-2001-00004

Revision 0

January 8, 200

Page 46 of 69

\begin{tabular}{|c|c|c|c|c|c|c|c|c|c|c|c|c|c|c|c|c|c|}
\hline Sample & File Name & \begin{tabular}{|l|} 
Count \\
Time
\end{tabular} & Area & $\% \sigma$ & $\sigma$ & cps & $\sigma$ cps & $1 / T$ & $\begin{array}{c}\text { Cf }= \\
\text { SQRT( }( \\
\text { 1/T) }\end{array}$ & $\begin{array}{c}\text { Corr. } \\
\text { cps }\end{array}$ & $\begin{array}{l}\text { Corr. } \\
\text { Sum } \\
\text { (cps) }\end{array}$ & \begin{tabular}{|c|} 
Drum \\
Activity \\
(dps)
\end{tabular} & $\begin{array}{c}\text { Drum } \\
\text { Activity } \\
\text { (nCi) }\end{array}$ & $\begin{array}{l}C_{\mathrm{f}} \text { of } \\
\ln 1 / \mathrm{T}\end{array}$ & $\begin{array}{l}\text { Corr. } \\
\text { Sum } \\
\text { (cps) }\end{array}$ & \begin{tabular}{|c|} 
Drum \\
Activity \\
(dps)
\end{tabular} & $\begin{array}{c}\text { Drum } \\
\text { Activity } \\
\text { (nCi) }\end{array}$ \\
\hline & T2573D1 & 157 & 1304 & 2.93 & 38.21 & 8.31 & 0.24 & & & & & & & & & & \\
\hline & T2573D3 & 157 & 492 & 4.93 & 24.26 & 3.13 & 0.15 & & & & & & & & & & \\
\hline & & & & & & & & & & & & & & & & & \\
\hline & T3573D1 & \begin{tabular}{|l|}
156.15 \\
\end{tabular} & 327 & 6.19 & 20.24 & 2.09 & 0.13 & & & & & & & & & & \\
\hline & & & & & & & & & & & & & & & & & \\
\hline & 573D1 & 670.24 & 106 & 14.23 & \begin{tabular}{|l|}
15.08 \\
\end{tabular} & 0.16 & 0.02 & & & 0.178 & & & & 0.17 & & & \\
\hline & 573D2 & 670.24 & 336 & 7.16 & 24.06 & 0.50 & 0.04 & & & 1.173 & 2.603 & 5551 & 149.88 & 0.91 & 2.19 & 4680 & 126 \\
\hline & 573D3 & 670.24 & 535 & 5.23 & 27.98 & 0.80 & 0.04 & & & 1.252 & & & & 1.11 & & & \\
\hline & & & & & & & & & & & & & & & & & \\
\hline FD405 & T1405D1 & 178.29 & 1516 & 2.72 & 41.24 & 8.50 & 0.23 & 1.91 & 1.383 & & & & & 1.28 & & & \\
\hline & T2405D2 & 192.34 & 317 & 8.42 & 26.69 & 1.65 & 0.14 & 10.01 & 3.164 & & & & & 2.16 & & & \\
\hline & T2405D3 & 192.34 & 517 & 4.89 & 25.28 & 2.69 & 0.13 & & & & & & & & & & \\
\hline & & & & & & & & & & & & & & & & & \\
\hline & T3405D1 & 300 & 239 & 7.47 & 17.85 & 0.80 & 0.06 & & & & & & & & & & \\
\hline & T3405D2 & 300 & 709 & 4.34 & 30.77 & 2.36 & 0.10 & & & & & & & & & & \\
\hline & T3405D3 & 300 & 564 & 4.68 & 26.40 & 1.88 & 0.09 & 8.89 & 2.981 & & & & & 2.09 & & & \\
\hline & & & & & & & & & & & & & & & & & \\
\hline & 405D1 & 1800 & 1 & 21155 & 211.55 & 0.00 & 0.12 & & & 8E-04 & & & & 0.00 & & & \\
\hline & 405D2 & 1800 & 40 & 49.78 & 19.91 & 0.02 & 0.01 & & & 0.07 & 0.217 & 462.28 & 12.482 & 0.05 & 0.15 & 322 & 9 \\
\hline & 405D3 & 1800 & 88 & 21.26 & 18.71 & 0.05 & 0.01 & & & 0.146 & & & & 0.10 & & & \\
\hline & & & & & & & & & & & & & & & & & \\
\hline & & & & & & & & & & & & & & & & & \\
\hline
\end{tabular}


WSRC-TR-2001-00004

Revision 0

January 8, 2001

Page 47 of 69

\begin{tabular}{|c|c|c|c|c|c|c|c|c|c|c|c|c|c|c|c|c|c|}
\hline Sample & $\begin{array}{c}\text { File } \\
\text { Name }\end{array}$ & $\begin{array}{l}\text { Count } \\
\text { Time }\end{array}$ & Area & $\% \sigma$ & $\sigma$ & cps & $\sigma$ cps & $1 / \mathbf{T}$ & \begin{tabular}{|c|} 
Cf $=$ \\
SQRT ( \\
1/T)
\end{tabular} & \begin{tabular}{|c|} 
Corr. \\
cps
\end{tabular} & $\begin{array}{l}\text { Corr. } \\
\text { Sum } \\
\text { (cps) }\end{array}$ & \begin{tabular}{|c|} 
Drum \\
Activity \\
(dps)
\end{tabular} & \begin{tabular}{|c|} 
Drum \\
Activity \\
(nCi)
\end{tabular} & $\begin{array}{l}C_{\mathrm{f}} \text { of } \\
\ln 1 / \mathrm{T}\end{array}$ & $\begin{array}{l}\text { Corr. } \\
\text { Sum } \\
\text { (cps) }\end{array}$ & \begin{tabular}{|c|} 
Drum \\
Activity \\
(dps)
\end{tabular} & $\begin{array}{c}\text { Drum } \\
\text { Activity } \\
\text { (nCi) }\end{array}$ \\
\hline \multirow[t]{10}{*}{ FD561 } & T1561D1 & 310.62 & 558 & 4.91 & 27.40 & 1.80 & 0.09 & 39.81 & 6.309 & & & & & 3.06 & & & \\
\hline & T1561D3 & 310.62 & 408 & 5.6 & 22.85 & 1.31 & 0.07 & & & & & & & & & & \\
\hline & & & & & & & & & & & & & & & & & \\
\hline & T2561D1 & 600 & 1194 & 3.19 & 38.09 & 1.99 & 0.06 & & & & & & & & & & \\
\hline & & & & & & & & & & & & & & & & & \\
\hline & T3561D1 & 449.84 & 794 & 4.06 & 32.24 & 1.77 & \begin{tabular}{|l|}
0.07 \\
\end{tabular} & & & & & & & & & & \\
\hline & T3561D2 & \begin{tabular}{|l|}
449.84 \\
\end{tabular} & 1443 & 2.93 & 42.28 & 3.21 & 0.09 & & & & & & & & & & \\
\hline & T3561D3 & 449.84 & 586 & 4.85 & 28.42 & 1.30 & 0.06 & 34.73 & \begin{tabular}{l|}
5.893 \\
\end{tabular} & & & & & 2.97 & & & \\
\hline & & & & & & & & & & & & & & & & & \\
\hline & 561D1 & 252.95 & 351 & 6.03 & 21.17 & 1.39 & 0.08 & & & 8.755 & & & & 4.25 & & & \\
\hline
\end{tabular}


WSRC-TR-2001-00004 Revision 0

January 8, 2001

Page 48 of 69

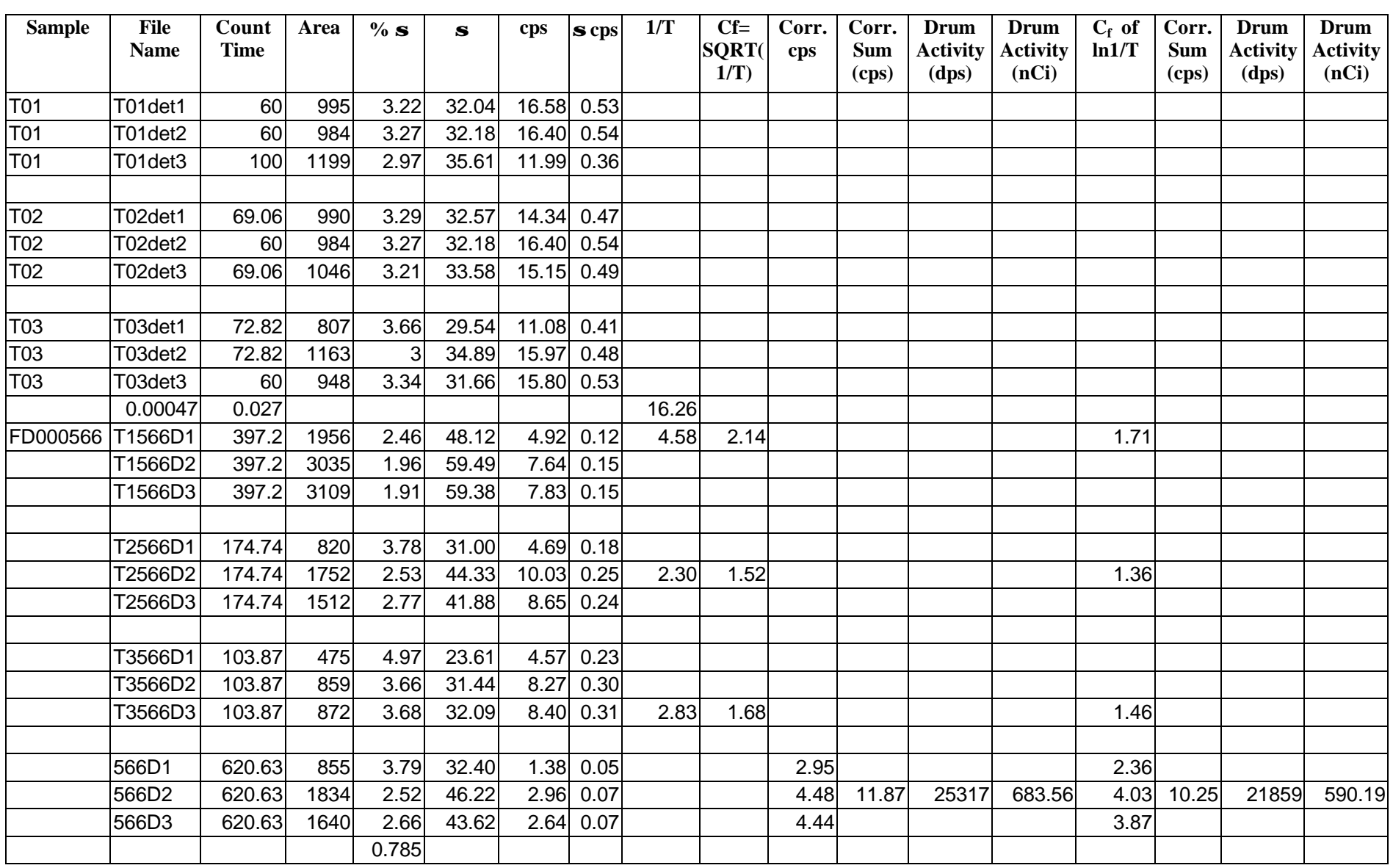


WSRC-TR-2001-00004

Revision 0

January 8, 2001

Page 49 of 69

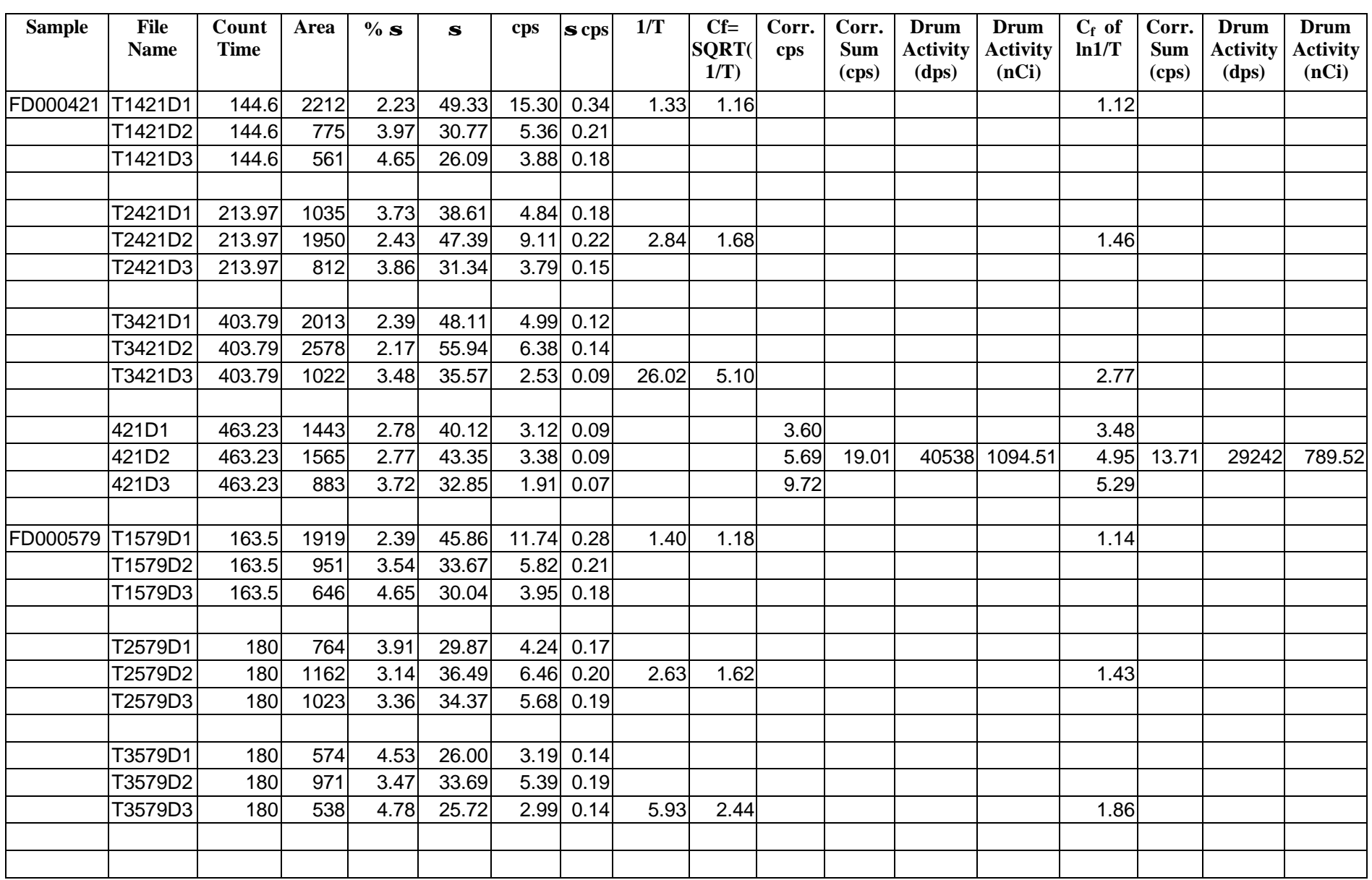


WSRC-TR-2001-00004

Revision 0

January 8, 200

Page 50 of 69

\begin{tabular}{|c|c|c|c|c|c|c|c|c|c|c|c|c|c|c|c|c|c|}
\hline Sample & $\begin{array}{c}\text { File } \\
\text { Name }\end{array}$ & $\begin{array}{c}\text { Count } \\
\text { Time }\end{array}$ & Area & $\% \sigma$ & $\sigma$ & cps & $\sigma$ cps & $1 / T$ & $\begin{array}{c}\text { Cf }= \\
\text { SQRT( }( \\
\text { 1/T) }\end{array}$ & $\begin{array}{c}\text { Corr. } \\
\text { cps }\end{array}$ & $\begin{array}{l}\text { Corr. } \\
\text { Sum } \\
\text { (cps) }\end{array}$ & \begin{tabular}{|c|} 
Drum \\
Activity \\
(dps)
\end{tabular} & $\begin{array}{c}\text { Drum } \\
\text { Activity } \\
\text { (nCi) }\end{array}$ & $\begin{array}{l}C_{\mathrm{f}} \text { of } \\
\ln 1 / \mathrm{T}\end{array}$ & \begin{tabular}{|l|} 
Corr. \\
Sum \\
(cps)
\end{tabular} & $\begin{array}{c}\text { Drum } \\
\text { Activity } \\
\text { (dps) }\end{array}$ & $\begin{array}{c}\text { Drum } \\
\text { Activity } \\
\text { (nCi) }\end{array}$ \\
\hline & 579D1 & 600 & 93 & 13.14 & 12.22 & 0.16 & 0.02 & & & 0.18 & & & & 0.18 & & & \\
\hline & 579D3 & 600 & 149 & 10.04 & 14.96 & 0.25 & 0.02 & & & 0.60 & & & & 0.46 & & & \\
\hline & & & & & & & & & & & & & & & & & \\
\hline \multirow[t]{9}{*}{\begin{tabular}{|l|} 
FD000582 \\
\end{tabular}} & T1582D1 & 94.05 & 1399 & 2.87 & 40.15 & 14.88 & 0.43 & 1.35 & 1.16 & & & & & 1.12 & & & \\
\hline & & & & & & & & & & & & & & & & & \\
\hline & T2582D1 & 222.52 & 1399 & 2.98 & 41.69 & 6.29 & 0.19 & & & & & & & & & & \\
\hline & T2582D2 & 222.52 & 2943 & 2.01 & 59.15 & 13.23 & 0.27 & 5.10 & 2.26 & & & & & 1.77 & & & \\
\hline & T2582D3 & 222.52 & 3020 & 2 & 60.40 & 13.57 & 0.27 & & & & & & & & & & \\
\hline & & & & & & & & & & & & & & & & & \\
\hline & T3582D1 & 165.88 & 782 & 4 & 31.28 & 4.71 & 0.19 & & & & & & & & & & \\
\hline & 582D2 & 120.96 & 1214 & 3.11 & 37.76 & 10.04 & 0.31 & & & 22.66 & 87.45 & \begin{tabular}{|l|}
186470 \\
\end{tabular} & \begin{tabular}{|l|}
5034.70 \\
\end{tabular} & 17.78 & 52.48 & 111888 & 3020.98 \\
\hline & 582D3 & 120.96 & 1306 & 3.03 & 39.57 & 10.80 & 0.33 & & & 61.55 & & & & 31.56 & & & \\
\hline & & & & & & & & & & & & & & & & & \\
\hline \multirow[t]{9}{*}{\begin{tabular}{|l} 
FD000569 \\
\end{tabular}} & T1569D1 & 300 & 3138 & 1.9 & 59.62 & 10.46 & 0.20 & 2.00 & 1.41 & & & & & 1.30 & & & \\
\hline & T1569D2 & 300 & 2905 & 1.97 & 57.23 & 9.68 & 0.19 & & & & & & & & & & \\
\hline & T1569D3 & 300 & 925 & 3.65 & 33.76 & 3.08 & 0.11 & & & & & & & & & & \\
\hline & & & & & & & & & & & & & & & & & \\
\hline & T2569D1 & 107.64 & 897 & 3.59 & 32.20 & 8.33 & 0.30 & & & & & & & & & & \\
\hline & T2569D2 & 107.64 & 597 & 4.42 & 26.39 & 5.55 & 0.25 & 5.30 & 2.30 & & & & & 1.79 & & & \\
\hline & T2569D3 & 107.64 & 796 & 3.73 & 29.69 & 7.40 & 0.28 & & & & & & & & & & \\
\hline & & & & & & & & & & & & & & & & & \\
\hline & & & & & & & & & & & & & & & & & \\
\hline
\end{tabular}


WSRC-TR-2001-00004

Revision 0

January 8, 200

Page 51 of 69

\begin{tabular}{|c|c|c|c|c|c|c|c|c|c|c|c|c|c|c|c|c|c|}
\hline Sample & $\begin{array}{c}\text { File } \\
\text { Name }\end{array}$ & $\begin{array}{l}\text { Count } \\
\text { Time }\end{array}$ & Area & $\% \sigma$ & $\sigma$ & cps & $\sigma$ cps & $\mathbf{1} / \mathbf{T}$ & $\begin{array}{c}\text { Cf }= \\
\text { SQRT( }( \\
\text { 1/T) }\end{array}$ & \begin{tabular}{|c|} 
Corr. \\
cps
\end{tabular} & $\begin{array}{l}\text { Corr. } \\
\text { Sum } \\
\text { (cps) }\end{array}$ & \begin{tabular}{|c|} 
Drum \\
Activity \\
(dps)
\end{tabular} & $\begin{array}{c}\text { Drum } \\
\text { Activity } \\
\text { (nCi) }\end{array}$ & $\begin{array}{l}C_{\mathrm{f}} \text { of } \\
\ln 1 / \mathrm{T}\end{array}$ & \begin{tabular}{|l|} 
Corr. \\
Sum \\
(cps)
\end{tabular} & \begin{tabular}{|c|} 
Drum \\
Activity \\
(dps)
\end{tabular} & $\begin{array}{c}\text { Drum } \\
\text { Activity } \\
\text { (nCi) }\end{array}$ \\
\hline & T3569D1 & 283.65 & 1311 & 2.94 & 38.54 & 4.62 & 0.14 & & & & & & & & & & \\
\hline & T3569D3 & 283.65 & 2013 & 2.41 & 48.51 & 7.10 & 0.17 & 3.47 & 1.86 & & & & & 1.57 & & & \\
\hline & & & & & & & & & & & & & & & & & \\
\hline & 569D1 & 577 & 1333 & 3 & 39.99 & 2.31 & \begin{tabular}{|l|}
0.07 \\
\end{tabular} & & & 3.26 & & & & 2.99 & & & \\
\hline & & & & & & & & & & & & & & & & & \\
\hline \multicolumn{18}{|c|}{ 10/05 acquisitions } \\
\hline T01 & T01det1 & 60 & 994 & 3.22 & 32.01 & 16.57 & 0.53 & & & & & & & & & & \\
\hline T01 & T01det2 & 60 & 984 & 3.27 & 32.18 & 16.40 & 0.54 & & & & & & & & & & \\
\hline \multirow[t]{2}{*}{ T01 } & T01det3 & 100 & 1199 & 2.97 & 35.61 & 11.99 & 0.36 & & & & & & & & & & \\
\hline & & & & & & & & & & & & & & & & & \\
\hline T03 & T03det1 & 72.82 & 807 & 3.66 & 29.54 & 11.08 & 0.41 & & & & & & & & & & \\
\hline T03 & T03det2 & 72.82 & 1163 & 3 & 34.89 & 15.97 & 0.48 & & & & & & & & & & \\
\hline \multirow[t]{2}{*}{ T03 } & T03det3 & 60 & 986 & 3.34 & 32.93 & 16.43 & 0.55 & & & & & & & & & & \\
\hline & 0.00047 & 0.027 & & & & & & 16.53 & & & & & & & & & \\
\hline & & & & & & & & & & & & & & & & & \\
\hline \multirow[t]{7}{*}{ FD000404 } & T1404D1 & 159.79 & 1995 & 2.34 & 46.68 & 12.49 & 0.29 & 1.36 & 1.17 & & & & & 1.13 & & & \\
\hline & T1404D2 & 159.79 & 1495 & 2.77 & 41.41 & 9.36 & 0.26 & & & & & & & & & & \\
\hline & T1404D3 & 159.79 & 158 & 9.62 & 15.20 & 0.99 & 0.10 & & & & & & & & & & \\
\hline & & & & & & & & & & & & & & & & & \\
\hline & T2404D1 & 321.56 & 2684 & 2.05 & 55.02 & 8.35 & 0.17 & & & & & & & & & & \\
\hline & T2404D2 & 321.56 & 1249 & 3.12 & 38.97 & 3.88 & 0.12 & 5.10 & 2.26 & & & & & 1.77 & & & \\
\hline & T2404D3 & 321.56 & 812 & 3.85 & 31.26 & 2.53 & 0.10 & & & & & & & & & & \\
\hline
\end{tabular}


WSRC-TR-2001-00004

Revision 0

January 8, 200

Page 52 of 69

\begin{tabular}{|c|c|c|c|c|c|c|c|c|c|c|c|c|c|c|c|c|c|}
\hline Sample & $\begin{array}{c}\text { File } \\
\text { Name }\end{array}$ & $\begin{array}{l}\text { Count } \\
\text { Time }\end{array}$ & Area & $\% \sigma$ & $\sigma$ & cps & $\sigma$ cps & $1 / T$ & $\begin{array}{c}\mathbf{C f}= \\
\text { SQRT( } \\
\text { 1/T) }\end{array}$ & $\begin{array}{c}\text { Corr. } \\
\text { cps }\end{array}$ & $\begin{array}{l}\text { Corr. } \\
\text { Sum } \\
\text { (cps) }\end{array}$ & \begin{tabular}{|c|} 
Drum \\
Activity \\
(dps)
\end{tabular} & $\begin{array}{c}\text { Drum } \\
\text { Activity } \\
\text { (nCi) }\end{array}$ & $\begin{array}{l}C_{\mathrm{f}} \text { of } \\
\ln 1 / \mathrm{T}\end{array}$ & \begin{tabular}{|l|} 
Corr. \\
Sum \\
(cps)
\end{tabular} & $\begin{array}{c}\text { Drum } \\
\text { Activity } \\
\text { (dps) }\end{array}$ & $\begin{array}{c}\text { Drum } \\
\text { Activity } \\
\text { (nCi) }\end{array}$ \\
\hline & T3404D1 & 301.06 & 566 & 4.81 & 27.22 & 1.88 & 0.09 & & & & & & & & & & \\
\hline & T3404D3 & 301.06 & 599 & 4.59 & 27.49 & 1.99 & 0.09 & 9.79 & 3.13 & & & & & 2.15 & & & \\
\hline & & & & & & & & & & & & & & & & & \\
\hline & 404D1 & 600 & 216 & 7.89 & 17.04 & 0.36 & 0.03 & & & 0.42 & & & & 0.41 & & & \\
\hline & & & & & & & & & & & & & & & & & \\
\hline \multirow[t]{11}{*}{ FD000384 } & T1384D1 & 143.04 & 1643 & 2.61 & 42.88 & 11.49 & 0.30 & 1.53 & 1.24 & & & & & 1.18 & & & \\
\hline & T1384D2 & 143.04 & 1791 & 2.49 & 44.60 & 12.52 & 0.31 & & & & & & & & & & \\
\hline & T1384D3 & 143.04 & 1069 & 3.27 & 34.96 & 7.47 & 0.24 & & & & & & & & & & \\
\hline & & & & & & & & & & & & & & & & & \\
\hline & T2384D1 & 141.76 & 1344 & 2.87 & 38.57 & 9.48 & 0.27 & & & & & & & & & & \\
\hline & T3384D2 & 241.54 & 733 & 4.01 & 29.39 & 3.03 & 0.12 & & & & & & & & & & \\
\hline & T3384D3 & 241.54 & 536 & 4.81 & 25.78 & 2.22 & 0.11 & -129.60 & $\mathrm{~N} / \mathrm{A}$ & & & & & $\mathrm{N} / \mathrm{A}$ & & & \\
\hline & & & & & & & & & & & & & & & & & \\
\hline & 384D1 & 600 & 409 & 5.75 & 23.52 & 0.68 & 0.04 & & & 0.84 & & & & 0.80 & & & \\
\hline & 384D2 & 600 & 927 & 3.66 & 33.93 & 1.55 & 0.06 & & & 2.08 & $\mathrm{~N} / \mathrm{A}$ & $\mathrm{N} / \mathrm{A}$ & $\mathrm{N} / \mathrm{A}$ & 1.93 & N/A & $\mathrm{N} / \mathrm{A}$ & $\mathrm{N} / \mathrm{A}$ \\
\hline & 384D3 & 600 & 1408 & 2.93 & 41.25 & 2.35 & 0.07 & & & $\mathrm{~N} / \mathrm{A}$ & & & & $\mathrm{N} / \mathrm{A}$ & & & \\
\hline & & & & & & & & & & & & & & & & & \\
\hline \multirow[t]{5}{*}{ FD000400 } & T1400D1 & 133.4 & 1480 & 2.7 & 39.96 & 11.09 & 0.30 & 1.51 & 1.23 & & & & & 1.17 & & & \\
\hline & T1400D2 & 133.4 & 1190 & 3.08 & 36.65 & 8.92 & 0.27 & & & & & & & & & & \\
\hline & T1400D3 & 133.4 & 539 & 4.64 & 25.01 & 4.04 & 0.19 & & & & & & & & & & \\
\hline & & & & & & & & & & & & & & & & & \\
\hline & & & & & & & & & & & & & & & & & \\
\hline
\end{tabular}


WSRC-TR-2001-00004

Revision 0

January 8, 200

Page 53 of 69

\begin{tabular}{|c|c|c|c|c|c|c|c|c|c|c|c|c|c|c|c|c|c|}
\hline Sample & $\begin{array}{c}\text { File } \\
\text { Name }\end{array}$ & $\begin{array}{c}\text { Count } \\
\text { Time }\end{array}$ & Area & $\% \sigma$ & $\sigma$ & cps & $\sigma \mathrm{cps}$ & $1 / \mathrm{T}$ & \begin{tabular}{|c|} 
Cf $=$ \\
SQRT( ( \\
1/T)
\end{tabular} & $\begin{array}{c}\text { Corr. } \\
\text { cps }\end{array}$ & $\begin{array}{l}\text { Corr. } \\
\text { Sum } \\
\text { (cps) }\end{array}$ & \begin{tabular}{|c|} 
Drum \\
Activity \\
(dps)
\end{tabular} & $\begin{array}{c}\text { Drum } \\
\text { Activity } \\
\text { (nCi) }\end{array}$ & $\begin{array}{l}C_{\mathrm{f}} \text { of } \\
\ln 1 / \mathrm{T}\end{array}$ & $\begin{array}{l}\text { Corr. } \\
\text { Sum } \\
\text { (cps) }\end{array}$ & \begin{tabular}{|c|} 
Drum \\
Activity \\
(dps)
\end{tabular} & \begin{tabular}{|c|} 
Drum \\
Activity \\
(nCi)
\end{tabular} \\
\hline & T2400D1 & 242.47 & 1780 & 2.53 & 45.03 & 7.34 & 0.19 & & & & & & & & & & \\
\hline & T2400D3 & 242.47 & 692 & 4.2 & 29.06 & 2.85 & 0.12 & & & & & & & & & & \\
\hline & & & & & & & & & & & & & & & & & \\
\hline & T3400D1 & 300.53 & 1118 & 3.25 & 36.34 & 3.72 & \begin{tabular}{|l|}
0.12 \\
\end{tabular} & & & & & & & & & & \\
\hline & & & & & & & & & & & & & & & & & \\
\hline & 400D1 & 600 & 82 & 14.19 & 11.64 & 0.14 & \begin{tabular}{|l|}
0.02 \\
\end{tabular} & & & 0.17 & & & & 0.16 & & & \\
\hline & $400 \mathrm{D} 2$ & 600 & 210 & 8.55 & 17.96 & 0.35 & 0.03 & & & 0.63 & 4.22 & 8995.75 & 242.89 & 0.53 & 2.87 & 6124.97 & 165.37 \\
\hline & 400D3 & 600 & 554 & 4.77 & 26.43 & 0.92 & \begin{tabular}{|l|}
0.04 \\
\end{tabular} & & & 3.42 & & & & 2.18 & & & \\
\hline FD000398 & T1398D1 & 113.87 & 1260 & 2.95 & 37.17 & 11.07 & 0.33 & 1.54 & 1.24 & & & & & 1.18 & & & \\
\hline & T2398D2 & 319.15 & 413 & 5.83 & 24.08 & 1.29 & 0.08 & 101.81 & 10.09 & & & & & 3.73 & & & \\
\hline & T2398D3 & 319.15 & 931 & 3.58 & 33.33 & 2.92 & 0.10 & & & & & & & & & & \\
\hline & & & & & & & & & & & & & & & & & \\
\hline & T3398D1 & 333.87 & 149 & 10.69 & 15.93 & 0.45 & \begin{tabular}{|l|}
0.05 \\
\end{tabular} & & & & & & & & & & \\
\hline & T3398D2 & 333.87 & 817 & 3.91 & 31.94 & 2.45 & 0.10 & & & & & & & & & & \\
\hline & T3398D3 & 333.87 & 964 & 3.52 & 33.93 & 2.89 & 0.10 & 10.00 & 3.16 & & & & & 2.16 & & & \\
\hline & & & & & & & & & & & & & & & & & \\
\hline & 398D1 & 600 & 186 & 8.98 & 16.70 & 0.31 & 0.03 & & & 0.38 & & & & 0.37 & & & \\
\hline & 398D2 & 600 & 679 & 4.31 & 29.26 & 1.13 & \begin{tabular}{|l|}
0.05 \\
\end{tabular} & & & 11.42 & 15.70 & 33480 & 903.97 & 4.22 & 7.25 & 15460 & 417.42 \\
\hline & 398D3 & 600 & 740 & 4.09 & 30.27 & 1.23 & \begin{tabular}{|l|}
0.05 \\
\end{tabular} & & & 3.90 & & & & 2.67 & & & \\
\hline & & & & & & & & & & & & & & & & & \\
\hline \multicolumn{2}{|c|}{ 10/09 Acquisitions } & & & & & & & & & & & & & & & & \\
\hline
\end{tabular}


WSRC-TR-2001-00004

Revision 0

January 8, 200

Page 54 of 69

\begin{tabular}{|c|c|c|c|c|c|c|c|c|c|c|c|c|c|c|c|c|c|}
\hline Sample & $\begin{array}{c}\text { File } \\
\text { Name }\end{array}$ & $\begin{array}{l}\text { Count } \\
\text { Time }\end{array}$ & Area & $\% \sigma$ & $\sigma$ & cps & $\sigma$ cps & $1 / T$ & \begin{tabular}{|c|}
$\mathbf{C f}=$ \\
SQRT( \\
1/T)
\end{tabular} & $\begin{array}{c}\text { Corr. } \\
\text { cps }\end{array}$ & $\begin{array}{l}\text { Corr. } \\
\text { Sum } \\
\text { (cps) }\end{array}$ & \begin{tabular}{|c|} 
Drum \\
Activity \\
(dps)
\end{tabular} & $\begin{array}{c}\text { Drum } \\
\text { Activity } \\
\text { (nCi) }\end{array}$ & $\begin{array}{l}C_{f} \text { of } \\
\ln 1 / T\end{array}$ & \begin{tabular}{|l|} 
Corr. \\
Sum \\
(cps)
\end{tabular} & \begin{tabular}{|c|} 
Drum \\
Activity \\
(dps)
\end{tabular} & $\begin{array}{c}\text { Drum } \\
\text { Activity } \\
\text { (nCi) }\end{array}$ \\
\hline T01 & T01det1 & 60 & 977 & 3.27 & 31.95 & 16.28 & \begin{tabular}{|l|}
0.53 \\
\end{tabular} & & & & & & & & & & \\
\hline T01 & T01det2 & 60 & 984 & 3.27 & 32.18 & 16.40 & 0.54 & & & & & & & & & & \\
\hline \multirow[t]{2}{*}{ T01 } & T01det3 & 100 & 1199 & 2.97 & 35.61 & 11.99 & 0.36 & & & & & & & & & & \\
\hline & & & & & & & & & & & & & & & & & \\
\hline T02 & T02det1 & 69.06 & 990 & 3.29 & 32.57 & 14.34 & 0.47 & & & & & & & & & & \\
\hline T02 & T02det2 & 60 & 963 & 3.33 & 32.07 & 16.05 & 0.53 & & & & & & & & & & \\
\hline \multirow[t]{2}{*}{ T02 } & T02det3 & 69.06 & 1046 & 3.21 & 33.58 & 15.15 & 0.49 & & & & & & & & & & \\
\hline & & & & & & & & & & & & & & & & & \\
\hline T03 & T03det1 & 72.82 & 807 & 3.66 & 29.54 & 11.08 & 0.41 & & & & & & & & & & \\
\hline T03 & T03det2 & 72.82 & 1163 & 3 & 34.89 & 15.97 & 0.48 & & & & & & & & & & \\
\hline \multirow[t]{2}{*}{ T03 } & T03det3 & 60 & 950 & 3.35 & 31.83 & 15.83 & 0.53 & & & & & & & & & & \\
\hline & 0.00047 & 0.027 & & & & & & 16.06 & & & & & & & & & \\
\hline & & & & & & & & & & & & & & & & & \\
\hline \multirow[t]{3}{*}{ FD000415 } & T1415D1 & 319.3 & 3780 & 1.71 & 64.64 & 11.84 & 0.20 & 1.38 & 1.17 & & & & & 1.13 & & & \\
\hline & T1415D2 & 319.3 & 829 & 4 & 33.16 & 2.60 & 0.10 & & & & & & & & & & \\
\hline & T1415D3 & 319.3 & 206 & 8.3 & 17.10 & 0.65 & 0.05 & & & & & & & & & & \\
\hline & & & & & & & & & & & & & & & & & \\
\hline & T2415D1 & 300 & 873 & 3.7 & 32.30 & 2.91 & 0.11 & & & & & & & & & & \\
\hline & T2415D2 & 300 & 467 & 5.24 & 24.47 & 1.56 & 0.08 & 14.71 & 3.84 & & & & & 2.40 & & & \\
\hline & T2415D3 & 300 & 428 & 5.52 & 23.63 & 1.43 & 0.08 & & & & & & & & & & \\
\hline & & & & & & & & & & & & & & & & & \\
\hline & T3415D1 & 300 & 250 & 7.53 & 18.83 & 0.83 & 0.06 & & & & & & & & & & \\
\hline & T3415D2 & 300 & 490 & 5.26 & 25.77 & 1.63 & 0.09 & & & & & & & & & & \\
\hline & T3415D3 & 300 & 508 & 5.12 & 26.01 & 1.69 & 0.09 & 11.50 & 3.39 & & & & & 2.25 & & & \\
\hline & & & & & & & & & & & & & & & & & \\
\hline & 415D1 & 3600 & 725 & 4.84 & 35.09 & 0.20 & 0.01 & & & 0.24 & & & & 0.23 & & & \\
\hline & 415D2 & 3600 & 1675 & 2.96 & 49.58 & 0.47 & 0.01 & & & 1.78 & 3.03 & \begin{tabular}{|l|}
6454.12 \\
\end{tabular} & 174.26 & \begin{tabular}{l|l|}
1.12 \\
\end{tabular} & 2.01 & \begin{tabular}{|l|}
4290.05 \\
\end{tabular} & 115.83 \\
\hline & 415D3 & 3600 & 1068 & 3.77 & 40.26 & 0.30 & 0.01 & & & 1.01 & & & & 0.67 & & & \\
\hline & & & & & & & & & & & & & & & & & \\
\hline
\end{tabular}


WSRC-TR-2001-00004

Revision 0

January 8, 200

Page 55 of 69

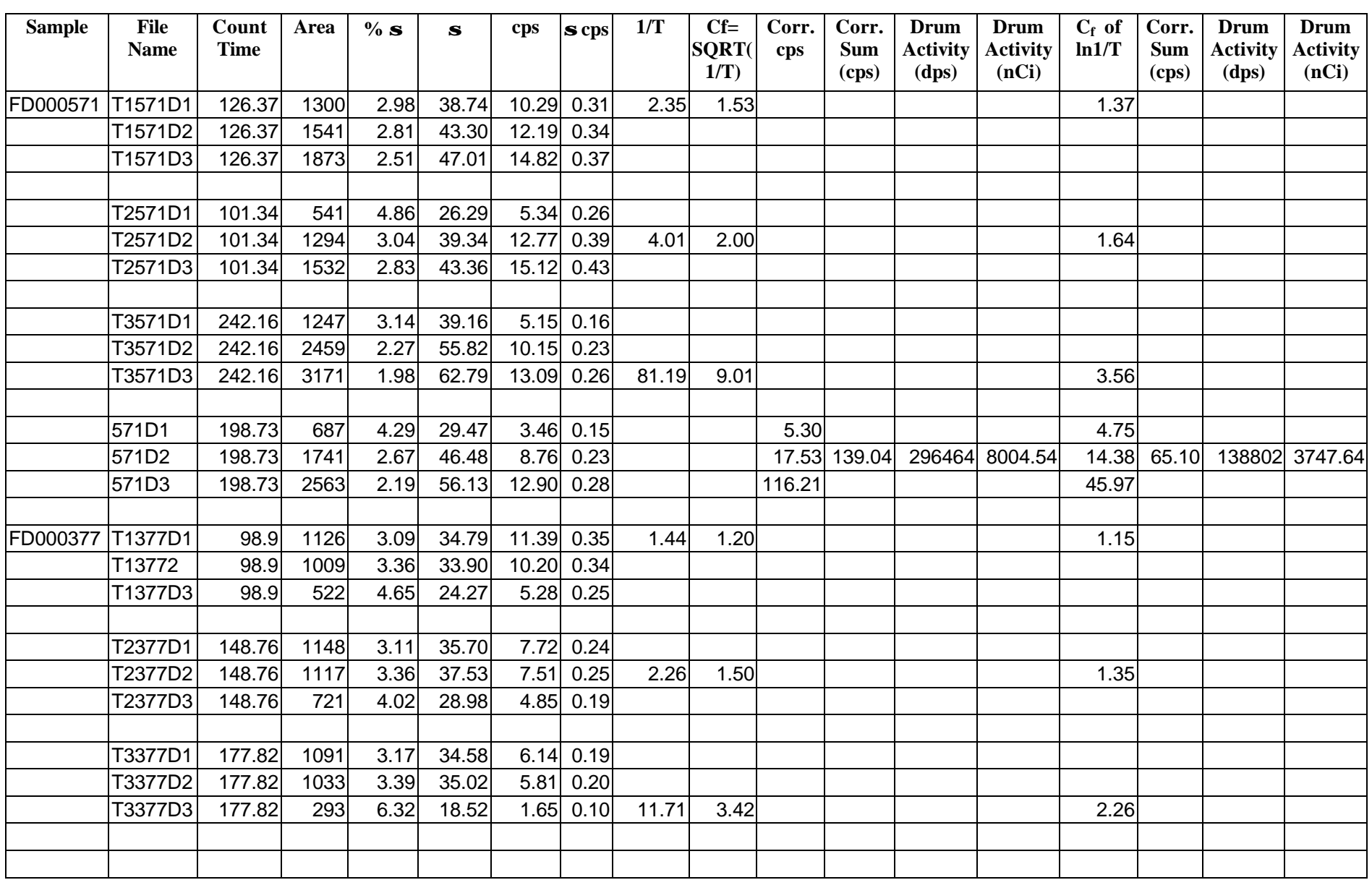


WSRC-TR-2001-00004 Revision 0

January 8, 2001 Page 56 of 69

\begin{tabular}{|c|c|c|c|c|c|c|c|c|c|c|c|c|c|c|c|c|c|}
\hline Sample & $\begin{array}{c}\text { File } \\
\text { Name }\end{array}$ & $\begin{array}{l}\text { Count } \\
\text { Time }\end{array}$ & Area & $\% \sigma$ & $\sigma$ & cps & $\sigma$ cps & $1 / T$ & $\begin{array}{c}\text { Cf }= \\
\text { SQRT( } \\
\text { 1/T) }\end{array}$ & $\begin{array}{c}\text { Corr. } \\
\text { cps }\end{array}$ & $\begin{array}{l}\text { Corr. } \\
\text { Sum } \\
\text { (cps) }\end{array}$ & $\begin{array}{c}\text { Drum } \\
\text { Activity } \\
\text { (dps) }\end{array}$ & $\begin{array}{c}\text { Drum } \\
\text { Activity } \\
\text { (nCi) }\end{array}$ & $\begin{array}{l}C_{\mathrm{f}} \text { of } \\
\ln 1 / \mathrm{T}\end{array}$ & $\begin{array}{l}\text { Corr. } \\
\text { Sum } \\
(\text { cps })\end{array}$ & \begin{tabular}{|c|} 
Drum \\
Activity \\
(dps)
\end{tabular} & $\begin{array}{c}\text { Drum } \\
\text { Activity } \\
\text { (nCi) }\end{array}$ \\
\hline & 377D1 & 351 & 77 & 13.25 & 10.20 & 0.22 & 0.03 & & & 0.26 & & & & 0.25 & & & \\
\hline & 377D3 & 351 & 97 & \begin{tabular}{|l|}
12.93 \\
\end{tabular} & \begin{tabular}{l|l}
12.54 \\
\end{tabular} & 0.28 & \begin{tabular}{|l|l|}
30.04 \\
\end{tabular} & & & 0.95 & & & & 0.62 & & & \\
\hline & & & & & & & & & & & & & & & & & \\
\hline \multirow[t]{9}{*}{\begin{tabular}{|l|} 
FD000411 \\
\end{tabular}} & T1411D1 & 95.02 & 1167 & 3.06 & 35.71 & 12.28 & 0.38 & 1.32 & 1.15 & & & & & 1.11 & & & \\
\hline & & & & & & & & & & & & & & & & & \\
\hline & T2411D1 & 89.25 & 522 & 4.69 & 24.48 & 5.85 & 0.27 & & & & & & & & & & \\
\hline & T2411D2 & 89.25 & 358 & 5.71 & 20.44 & 4.01 & 0.23 & 4.31 & 2.08 & & & & & 1.68 & & & \\
\hline & T2411D3 & 89.25 & 503 & 4.89 & 24.60 & 5.64 & 0.28 & & & & & & & & & & \\
\hline & & & & & & & & & & & & & & & & & \\
\hline & T3411D1 & 152.98 & 343 & 5.96 & 20.44 & 2.24 & 0.13 & & & & & & & & & & \\
\hline & 411D2 & 1000 & 287 & 7.52 & 21.58 & 0.29 & 0.02 & & & 0.60 & 1.15 & 2446.32 & 66.05 & 0.48 & 0.92 & \begin{tabular}{|l|}
1969.06 \\
\end{tabular} & 53.16 \\
\hline & 411D3 & 1000 & 188 & 10.45 & $\begin{array}{l}19.65 \\
\end{array}$ & 0.19 & 0.02 & & & 0.46 & & & & 0.35 & & & \\
\hline & & & & & & & & & & & & & & & & & \\
\hline \multirow[t]{7}{*}{\begin{tabular}{|l|} 
FD000389 \\
\end{tabular}} & T1389D1 & 116.23 & 1408 & 5.63 & 79.27 & 12.11 & 0.68 & 1.60 & 1.27 & & & & & 1.20 & & & \\
\hline & T1389D2 & 116.53 & 465 & 5.27 & 24.51 & 3.99 & 0.21 & & & & & & & & & & \\
\hline & T1389D3 & 116.23 & 193 & 8.44 & 16.29 & 1.66 & \begin{tabular}{|l|}
0.14 \\
\end{tabular} & & & & & & & & & & \\
\hline & & & & & & & & & & & & & & & & & \\
\hline & T2389D1 & 170.94 & 751 & 3.98 & 29.89 & 4.39 & \begin{tabular}{|l|}
0.17 \\
\end{tabular} & & & & & & & & & & \\
\hline & T2389D2 & 170.94 & 565 & 4.87 & 27.52 & 3.31 & 0.16 & 189.59 & 13.77 & & & & & 4.19 & & & \\
\hline & T2389D3 & 170.94 & 328 & \begin{tabular}{l|l}
6.3 \\
\end{tabular} & 20.66 & 1.92 & \begin{tabular}{|l|}
0.12 \\
\end{tabular} & & & & & & & & & & \\
\hline & & & & & & & & & & & & & & & & & \\
\hline & & & & & & & & & & & & & & & & & \\
\hline
\end{tabular}


WSRC-TR-2001-00004

Revision 0

January 8, 200

Page 57 of 69

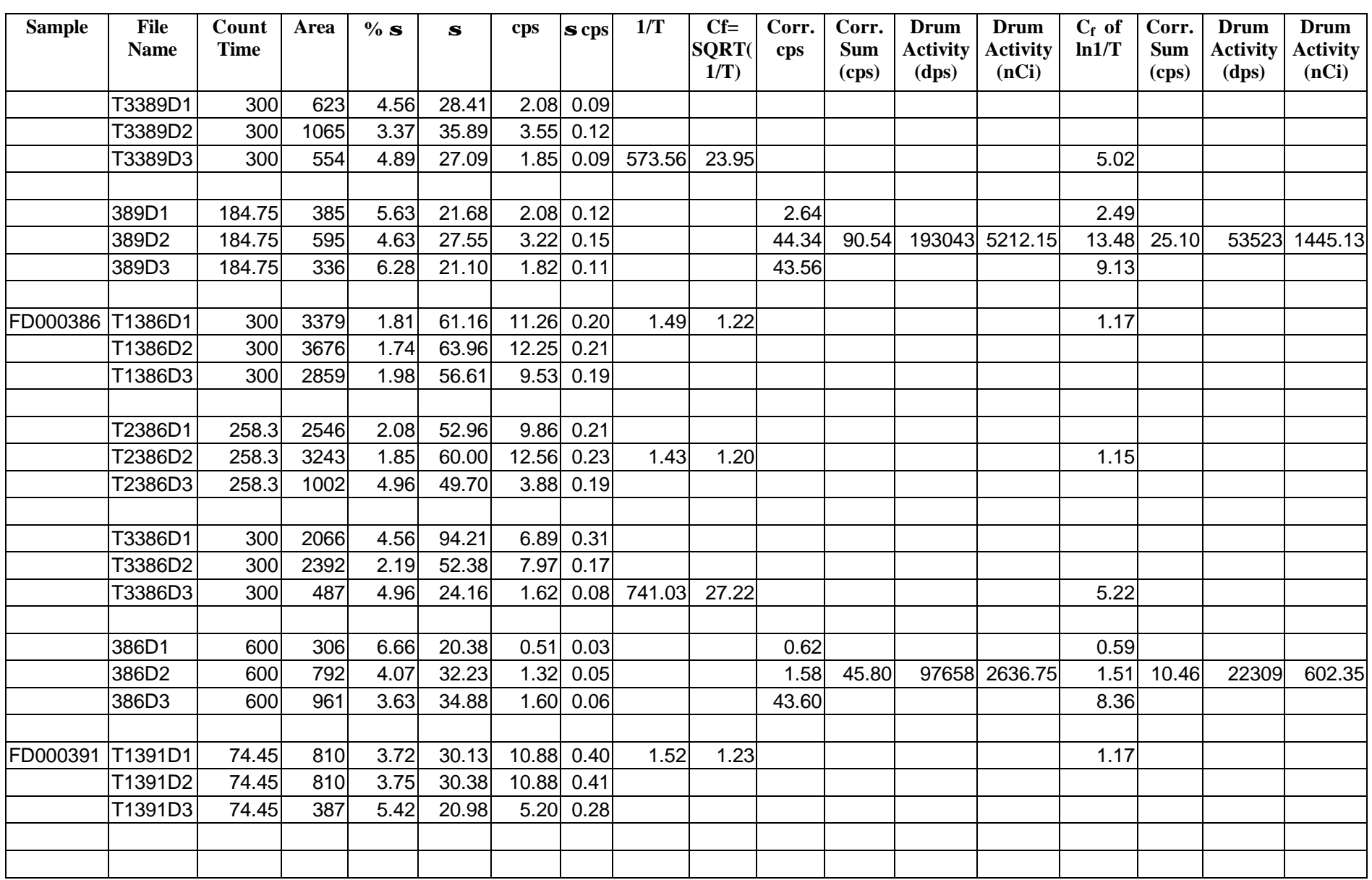


WSRC-TR-2001-00004

Revision 0

January 8, 2001

Page 58 of 69

\begin{tabular}{|c|c|c|c|c|c|c|c|c|c|c|c|c|c|c|c|c|c|}
\hline Sample & $\begin{array}{c}\text { File } \\
\text { Name }\end{array}$ & $\begin{array}{l}\text { Count } \\
\text { Time }\end{array}$ & Area & $\% \sigma$ & $\sigma$ & cps & $\sigma$ cps & $1 / T$ & \begin{tabular}{|c|} 
Cf $=$ \\
SQRT( ( \\
1/T)
\end{tabular} & $\begin{array}{c}\text { Corr. } \\
\text { cps }\end{array}$ & $\begin{array}{l}\text { Corr. } \\
\text { Sum } \\
\text { (cps) }\end{array}$ & $\begin{array}{c}\text { Drum } \\
\text { Activity } \\
\text { (dps) }\end{array}$ & $\begin{array}{c}\text { Drum } \\
\text { Activity } \\
\text { (nCi) }\end{array}$ & $\begin{array}{l}C_{f} \text { of } \\
\ln 1 / T\end{array}$ & $\begin{array}{l}\text { Corr. } \\
\text { Sum } \\
\text { (cps) }\end{array}$ & $\begin{array}{c}\text { Drum } \\
\text { Activity } \\
\text { (dps) }\end{array}$ & $\begin{array}{c}\text { Drum } \\
\text { Activity } \\
\text { (nCi) }\end{array}$ \\
\hline & T2391D1 & 90.82 & 834 & 3.64 & 30.36 & 9.18 & \begin{tabular}{|l|l|}
0.33 \\
\end{tabular} & & & & & & & & & & \\
\hline & T2391D3 & 90.82 & 132 & 10 & 13.20 & 1.45 & 0.15 & & & & & & & & & & \\
\hline & & & & & & & & & & & & & & & & & \\
\hline & T3391D1 & 360.76 & 1843 & 2.5 & 46.08 & 5.11 & \begin{tabular}{|l|}
0.13 \\
\end{tabular} & & & & & & & & & & \\
\hline & & & & & & & & & & & & & & & & & \\
\hline & 391D1 & 590.85 & 199 & 8.64 & 17.19 & 0.34 & \begin{tabular}{|l|}
0.03 \\
\end{tabular} & & & 0.42 & & & & 0.40 & & & \\
\hline & 391D2 & 590.85 & 480 & 5.48 & 26.30 & 0.81 & 0.04 & & & 1.08 & 10.53 & 22455 & 606.28 & 1.00 & 5.43 & 11588 & 312.87 \\
\hline & 391D3 & 590.85 & 729 & 4.19 & 30.55 & 1.23 & 0.05 & & & 9.04 & & & & 4.03 & & & \\
\hline & & & & & & & & & & & & & & & & & \\
\hline \multicolumn{18}{|c|}{ 10/10 Acquisitions } \\
\hline T03 & T03det1 & 43.38 & 686 & 3.95 & 27.10 & 15.81 & \begin{tabular}{|l|}
0.62 \\
\end{tabular} & & & & & & & & & & \\
\hline & \begin{tabular}{|l|}
0.00047 \\
\end{tabular} & 0.027 & & & & & & 16.09 & & & & & & & & & \\
\hline & & & & & & & & & & & & & & & & & \\
\hline FD000390 & T1390D1 & 313.67 & 3644 & 1.76 & 64.13 & 11.62 & 0.20 & 1.48 & 1.22 & & & & & 1.16 & & & \\
\hline & T1390D2 & 313.67 & 3267 & 1.94 & 63.38 & 10.42 & \begin{tabular}{|l|}
0.20 \\
\end{tabular} & & & & & & & & & & \\
\hline & T1390D3 & 313.67 & 1151 & 3.77 & 43.39 & 3.67 & 0.14 & & & & & & & & & & \\
\hline & & & & & & & & & & & & & & & & & \\
\hline & T2390D1 & 490.05 & 3767 & 1.76 & 66.30 & 7.69 & 0.14 & & & & & & & & & & \\
\hline & T2390D2 & 490.05 & 3938 & 1.77 & 69.70 & 8.04 & 0.14 & 2.66 & 1.63 & & & & & 1.43 & & & \\
\hline & T2390D3 & 490.05 & 1367 & 3.56 & 48.67 & 2.79 & 0.10 & & & & & & & & & & \\
\hline & & & & & & & & & & & & & & & & & \\
\hline & & & & & & & & & & & & & & & & & \\
\hline
\end{tabular}


WSRC-TR-2001-00004

Revision 0

January 8, 200

Page 59 of 69

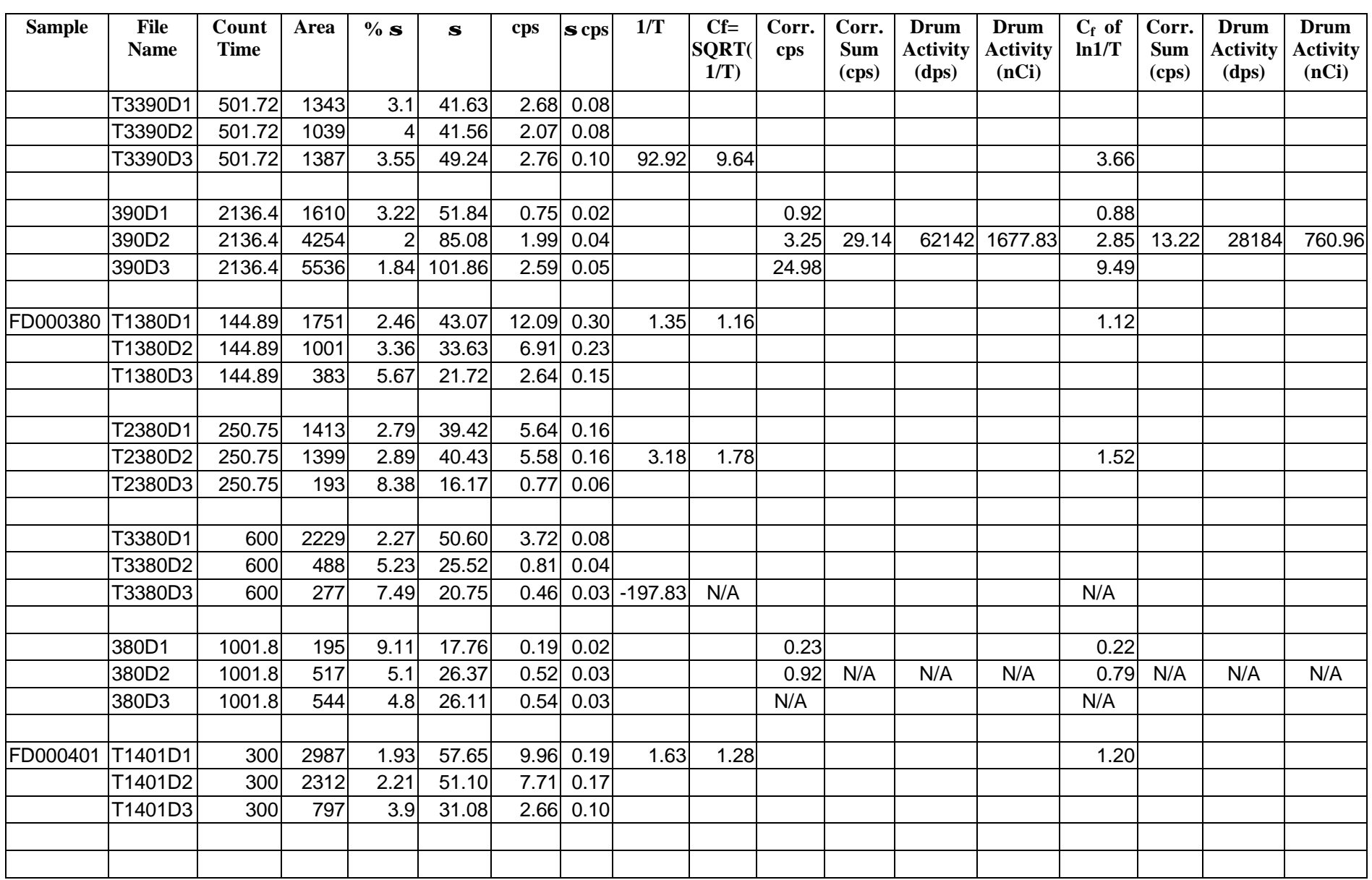


WSRC-TR-2001-00004

Revision 0

January 8, 2001

Page 60 of 69

\begin{tabular}{|c|c|c|c|c|c|c|c|c|c|c|c|c|c|c|c|c|c|}
\hline \multirow[t]{3}{*}{ Sample } & $\begin{array}{c}\text { File } \\
\text { Name }\end{array}$ & $\begin{array}{c}\text { Count } \\
\text { Time }\end{array}$ & Area & $\% \sigma$ & $\sigma$ & cps & $\sigma$ cps & $1 / T$ & $\begin{array}{c}\text { Cf }= \\
\text { SQRT( }( \\
\text { 1/T) }\end{array}$ & $\begin{array}{c}\text { Corr. } \\
\text { cps }\end{array}$ & $\begin{array}{l}\text { Corr. } \\
\text { Sum } \\
\text { (cps) }\end{array}$ & \begin{tabular}{|c|} 
Drum \\
Activity \\
(dps)
\end{tabular} & $\begin{array}{c}\text { Drum } \\
\text { Activity } \\
\text { (nCi) }\end{array}$ & $\begin{array}{l}C_{\mathrm{f}} \text { of } \\
\ln 1 / \mathrm{T}\end{array}$ & $\begin{array}{l}\text { Corr. } \\
\text { Sum } \\
\text { (cps) }\end{array}$ & $\begin{array}{c}\text { Drum } \\
\text { Activity } \\
\text { (dps) }\end{array}$ & \multirow{2}{*}{\begin{tabular}{|c}
$\begin{array}{c}\text { Drum } \\
\text { Activity } \\
\text { (nCi) }\end{array}$ \\
\end{tabular}} \\
\hline & T2401D1 & 300 & 1872 & 2.46 & 46.05 & 6.24 & 0.15 & & & & & & & & & & \\
\hline & T2401D3 & 300 & 447 & 5.45 & 24.36 & 1.49 & 0.08 & & & & & & & & & & \\
\hline & & & & & & & & & & & & & & & & & \\
\hline & T3401D1 & 300 & 1010 & 3.39 & 34.24 & 3.37 & 0.11 & & & & & & & & & & \\
\hline & & & & & & & & & & & & & & & & & \\
\hline & 401D1 & 600 & 51 & 20.28 & 10.34 & 0.09 & 0.02 & & & 0.11 & & & & 0.10 & & & \\
\hline & 401D2 & 600 & 151 & 9.9 & 14.95 & 0.25 & 0.02 & & & 0.52 & 1.50 & 3205.35 & 86.54 & 0.42 & 1.01 & 2160.98 & 58.35 \\
\hline & 401D3 & 600 & 109 & 12.57 & 13.70 & 0.18 & 0.02 & & & 0.88 & & & & 0.49 & & & \\
\hline & & & & & & & & & & & & & & & & & \\
\hline FD000381 & T1381D1 & 146.14 & 1612 & 2.62 & 42.23 & 11.03 & 0.29 & 1.47 & 1.21 & & & & & 1.16 & & & \\
\hline & T2381D2 & 190.81 & 1134 & 3.14 & 35.61 & 5.94 & 0.19 & 2.83 & 1.68 & & & & & 1.46 & & & \\
\hline & T2381D3 & 190.81 & 170 & 8.94 & 15.20 & 0.89 & 0.08 & & & & & & & & & & \\
\hline & & & & & & & & & & & & & & & & & \\
\hline & T3381D1 & 300 & 1177 & 3.09 & 36.37 & 3.92 & 0.12 & & & & & & & & & & \\
\hline & T3381D2 & 300 & 171 & 9.57 & 16.36 & 0.57 & 0.05 & & & & & & & & & & \\
\hline & T3381D3 & 300 & 136 & 10.76 & 14.63 & 0.45 & 0.05 & 111.92 & 10.58 & & & & & 3.80 & & & \\
\hline & & & & & & & & & & & & & & & & & \\
\hline & 381D1 & 1757.5 & 182 & 9.97 & 18.15 & 0.10 & 0.01 & & & 0.13 & & & & 0.12 & & & \\
\hline & 381D2 & 1757.5 & 443 & 5.73 & 25.38 & 0.25 & 0.01 & & & 0.42 & 3.82 & \begin{tabular}{|l|}
8153.98 \\
\end{tabular} & 220.16 & 0.37 & 1.66 & 3548.31 & 95.80 \\
\hline & 381D3 & 1757.5 & 544 & 5.14 & 27.96 & 0.31 & 0.02 & & & 3.27 & & & & 1.18 & & & \\
\hline & & & & & & & & & & & & & & & & & \\
\hline & & & & & & & & & & & & & & & & & \\
\hline
\end{tabular}


WSRC-TR-2001-00004

Revision 0

January 8, 2001

Page 61 of 69

\begin{tabular}{|c|c|c|c|c|c|c|c|c|c|c|c|c|c|c|c|c|c|}
\hline Sample & $\begin{array}{c}\text { File } \\
\text { Name }\end{array}$ & $\begin{array}{l}\text { Count } \\
\text { Time }\end{array}$ & Area & $\% \sigma$ & $\sigma$ & cps & $\sigma$ cps & $1 / T$ & \begin{tabular}{|c|} 
Cf $=$ \\
SQRT( \\
1/T)
\end{tabular} & $\begin{array}{c}\text { Corr. } \\
\text { cps }\end{array}$ & $\begin{array}{l}\text { Corr. } \\
\text { Sum } \\
\text { (cps) }\end{array}$ & $\begin{array}{c}\text { Drum } \\
\text { Activity } \\
\text { (dps) }\end{array}$ & $\begin{array}{c}\text { Drum } \\
\text { Activity } \\
\text { (nCi) }\end{array}$ & $\begin{array}{l}C_{f} \text { of } \\
\ln 1 / T\end{array}$ & $\begin{array}{l}\text { Corr. } \\
\text { Sum } \\
\text { (cps) }\end{array}$ & \begin{tabular}{|c|} 
Drum \\
Activity \\
(dps)
\end{tabular} & $\begin{array}{c}\text { Drum } \\
\text { Activity } \\
\text { (nCi) }\end{array}$ \\
\hline \multirow[t]{15}{*}{ FD000392 } & T1392D1 & 110.11 & 1394 & 2.85 & 39.73 & 12.66 & 0.36 & 1.59 & 1.26 & & & & & 1.19 & & & \\
\hline & T1392D2 & 110.11 & 1044 & 3.38 & 35.29 & 9.48 & 0.32 & & & & & & & & & & \\
\hline & T1392D3 & 110.11 & 894 & 3.7 & 33.08 & 8.12 & 0.30 & & & & & & & & & & \\
\hline & & & & & & & & & & & & & & & & & \\
\hline & T2392D1 & 68.63 & 409 & 5.41 & 22.13 & 5.96 & 0.32 & & & & & & & & & & \\
\hline & T2392D2 & 68.63 & 734 & 4 & 29.36 & 10.70 & 0.43 & 3.37 & 1.83 & & & & & 1.55 & & & \\
\hline & T2392D3 & 68.63 & 667 & 4.38 & 29.21 & 9.72 & 0.43 & & & & & & & & & & \\
\hline & & & & & & & & & & & & & & & & & \\
\hline & T3392D1 & 149.01 & 601 & 4.44 & 26.68 & 4.03 & 0.18 & & & & & & & & & & \\
\hline & T3392D2 & 149.01 & 1463 & 2.85 & 41.70 & 9.82 & 0.28 & & & & & & & & & & \\
\hline & T3392D3 & 149.01 & 1091 & 3.4 & 37.09 & 7.32 & 0.25 & 16.15 & \begin{tabular}{l|l}
4.02 \\
\end{tabular} & & & & & 2.46 & & & \\
\hline & & & & & & & & & & & & & & & & & \\
\hline & 392D1 & 517.31 & 1303 & 3.06 & 39.87 & 2.52 & 0.08 & & & 3.17 & & & & 3.00 & & & \\
\hline & 329D2 & 517.31 & 3060 & 2 & 61.20 & 5.92 & 0.12 & & & 10.85 & 39.44 & 84102 & 2270.75 & 9.17 & 27.74 & 59153 & 1597.13 \\
\hline & 392D3 & 517.31 & 3272 & \begin{tabular}{l|}
1.95 \\
\end{tabular} & 63.80 & 6.33 & 0.12 & & & 25.42 & & & & 15.56 & & & \\
\hline & & & & & & & & & & & & & & & & & \\
\hline \multicolumn{18}{|c|}{ 10/11 Acquisitions } \\
\hline \multirow[t]{2}{*}{ T01 } & T01det1 & 60 & 989 & 3.27 & 32.34 & 16.48 & 0.54 & & & & & & & & & & \\
\hline & & & & & & & & & & & & & & & & & \\
\hline \multirow[t]{2}{*}{ T02 } & T02det1 & 60 & 1040 & 3.16 & 32.86 & 17.33 & 0.55 & & & & & & & & & & \\
\hline & & & & & & & & & & & & & & & & & \\
\hline \multirow[t]{2}{*}{ T03 } & T03det1 & 60 & 973 & 3.4 & 33.08 & 16.22 & 0.55 & & & & & & & & & & \\
\hline & 0.00047 & 0.027 & & & & & & 16.68 & & & & & & & & & \\
\hline & & & & & & & & & & & & & & & & & \\
\hline \multirow[t]{5}{*}{ FD000387 } & T1387D1 & 133.37 & 2087 & 2.34 & 48.84 & 15.65 & 0.37 & 1.37 & 1.17 & & & & & 1.13 & & & \\
\hline & T1387D2 & 133.37 & 3037 & 1.99 & 60.44 & 22.77 & 0.45 & & & & & & & & & & \\
\hline & T1387D3 & 133.37 & 1611 & 2.83 & 45.59 & 12.08 & 0.34 & & & & & & & & & & \\
\hline & & & & & & & & & & & & & & & & & \\
\hline & & & & & & & & & & & & & & & & & \\
\hline
\end{tabular}


WSRC-TR-2001-00004

Revision 0

January 8, 200

Page 62 of 69

\begin{tabular}{|c|c|c|c|c|c|c|c|c|c|c|c|c|c|c|c|c|c|}
\hline Sample & $\begin{array}{c}\text { File } \\
\text { Name }\end{array}$ & $\begin{array}{l}\text { Count } \\
\text { Time }\end{array}$ & Area & $\% \sigma$ & $\sigma$ & cps & $\sigma$ cps & $1 / T$ & \begin{tabular}{|c|} 
Cf $=$ \\
SQRT( ( \\
1/T)
\end{tabular} & $\begin{array}{c}\text { Corr. } \\
\text { cps }\end{array}$ & $\begin{array}{l}\text { Corr. } \\
\text { Sum } \\
\text { (cps) }\end{array}$ & \begin{tabular}{|c|} 
Drum \\
Activity \\
(dps)
\end{tabular} & \begin{tabular}{|c|} 
Drum \\
Activity \\
(nCi)
\end{tabular} & $\begin{array}{l}C_{f} \text { of } \\
\ln 1 / T\end{array}$ & $\begin{array}{l}\text { Corr. } \\
\text { Sum } \\
\text { (cps) }\end{array}$ & \begin{tabular}{|c|} 
Drum \\
Activity \\
(dps)
\end{tabular} & \begin{tabular}{|c|} 
Drum \\
Activity \\
(nCi)
\end{tabular} \\
\hline & T2387D1 & 136.32 & 1787 & 2.54 & 45.39 & 13.11 & 0.33 & & & & & & & & & & \\
\hline & T2387D3 & 136.32 & 1698 & 2.75 & 46.70 & 12.46 & 0.34 & & & & & & & & & & \\
\hline & & & & & & & & & & & & & & & & & \\
\hline & T3387D1 & 268.04 & 1024 & 3.64 & 37.27 & 3.82 & 0.14 & & & & & & & & & & \\
\hline & & & & & & & & & & & & & & & & & \\
\hline & 387D1 & 3600 & 12398 & 1.06 & 131.42 & 3.44 & 0.04 & & & 4.03 & & & & 3.88 & & & \\
\hline & 387D2 & 3600 & 44682 & 0.54 & 241.28 & 12.41 & \begin{tabular}{|l|}
0.07 \\
\end{tabular} & & & 34.00 & $\mathrm{~N} / \mathrm{A}$ & $\mathrm{N} / \mathrm{A}$ & $\mathrm{N} / \mathrm{A}$ & 24.72 & $\mathrm{~N} / \mathrm{A}$ & $\mathrm{N} / \mathrm{A}$ & $\mathrm{N} / \mathrm{A}$ \\
\hline & 387D3 & 3600 & 43061 & 0.55 & 236.84 & 11.96 & 0.07 & & & $\mathrm{~N} / \mathrm{A}$ & & & & $\mathrm{N} / \mathrm{A}$ & & & \\
\hline & & & & & & & & & & & & & & & & & \\
\hline FD000395 & T1395D1 & 101.45 & 1143 & 3.11 & 35.55 & 11.27 & 0.35 & 1.66 & 1.29 & & & & & 1.21 & & & \\
\hline & T2395D2 & 300 & 706 & 4.3 & 30.36 & 2.35 & 0.10 & 32.36 & 5.69 & & & & & 2.92 & & & \\
\hline & T2395D3 & 300 & 1549 & 2.78 & 43.06 & 5.16 & 0.14 & & & & & & & & & & \\
\hline & & & & & & & & & & & & & & & & & \\
\hline & T3395D1 & 222.44 & 294 & 6.53 & 19.20 & 1.32 & 0.09 & & & & & & & & & & \\
\hline & T3395D2 & 222.44 & 861 & 3.81 & 32.80 & 3.87 & 0.15 & & & & & & & & & & \\
\hline & T3395D3 & 222.44 & 854 & 3.87 & 33.05 & 3.84 & 0.15 & 19.86 & 4.46 & & & & & 2.59 & & & \\
\hline & & & & & & & & & & & & & & & & & \\
\hline & 395D1 & 523.43 & 653 & 4.3 & 28.08 & 1.25 & 0.05 & & & 1.61 & & & & \begin{tabular}{l|l|}
1.51 \\
\end{tabular} & & & \\
\hline & 395D2 & 523.43 & 962 & 3.81 & 36.65 & 1.84 & 0.07 & & & 10.45 & 25.43 & 54223 & \begin{tabular}{|l|}
1464.01 \\
\end{tabular} & 5.37 & 14.66 & 31262 & 844.08 \\
\hline & 395D3 & 523.43 & 1570 & 2.81 & \begin{tabular}{l|}
44.12 \\
\end{tabular} & 3.00 & 0.08 & & & 13.37 & & & & 7.78 & & & \\
\hline & & & & & & & & & & & & & & & & & \\
\hline & & & & & & & & & & & & & & & & & \\
\hline
\end{tabular}


WSRC-TR-2001-00004

Revision 0

January 8, 2001

Page 63 of 69

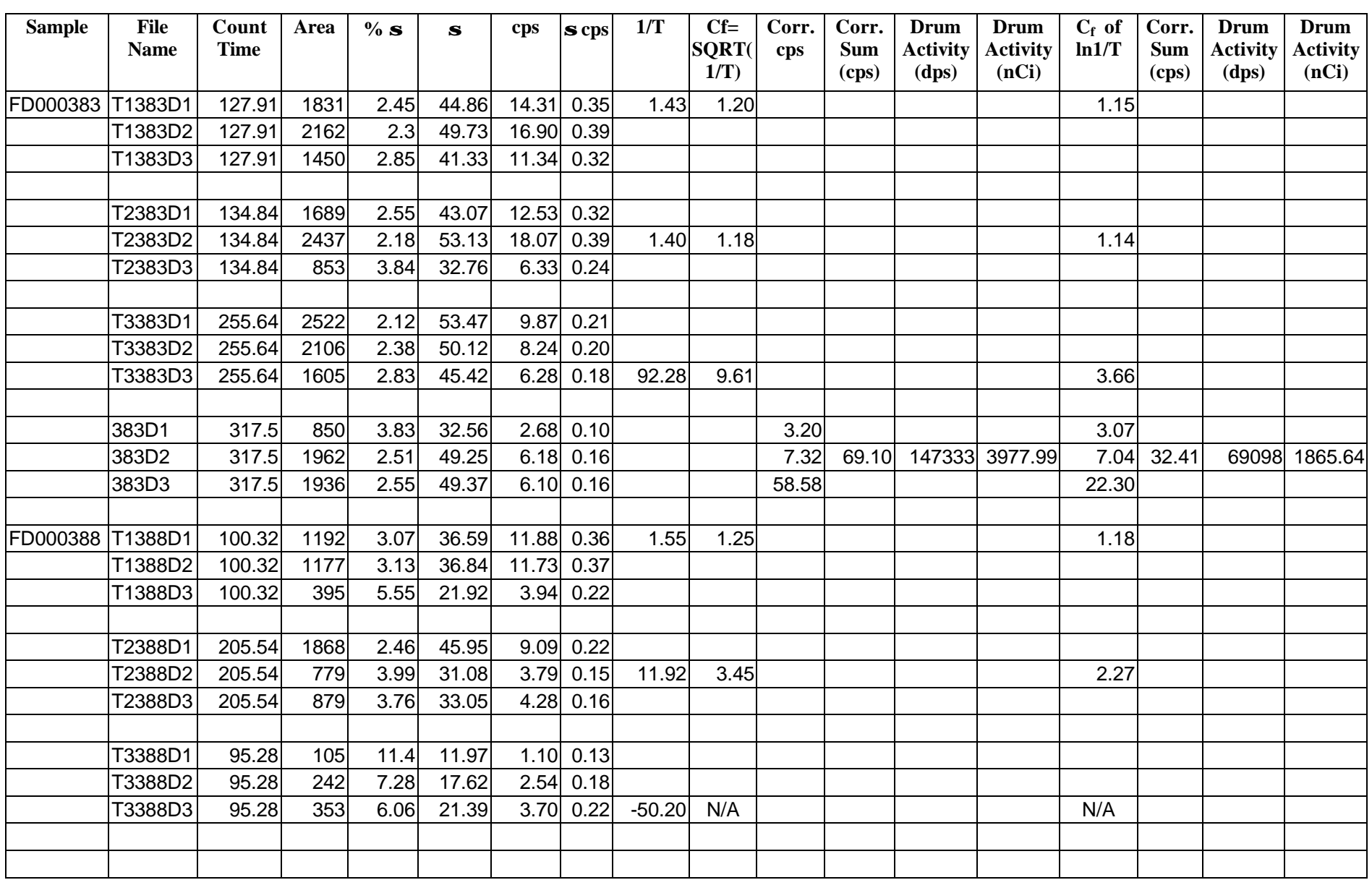


WSRC-TR-2001-00004

Revision 0

January 8, 200

Page 64 of 69

\begin{tabular}{|c|c|c|c|c|c|c|c|c|c|c|c|c|c|c|c|c|c|}
\hline Sample & $\begin{array}{c}\text { File } \\
\text { Name }\end{array}$ & $\begin{array}{l}\text { Count } \\
\text { Time }\end{array}$ & Area & $\% \sigma$ & $\sigma$ & cps & $\sigma$ cps & $1 / T$ & $\begin{array}{c}\text { Cf }= \\
\text { SQRT( }( \\
\text { 1/T) }\end{array}$ & $\begin{array}{c}\text { Corr. } \\
\text { cps }\end{array}$ & $\begin{array}{l}\text { Corr. } \\
\text { Sum } \\
\text { (cps) }\end{array}$ & \begin{tabular}{|c|} 
Drum \\
Activity \\
(dps)
\end{tabular} & $\begin{array}{c}\text { Drum } \\
\text { Activity } \\
\text { (nCi) }\end{array}$ & $\begin{array}{l}C_{\mathrm{f}} \text { of } \\
\ln 1 / \mathrm{T}\end{array}$ & \begin{tabular}{|l|} 
Corr. \\
Sum \\
(cps)
\end{tabular} & \begin{tabular}{|c|} 
Drum \\
Activity \\
(dps)
\end{tabular} & $\begin{array}{c}\text { Drum } \\
\text { Activity } \\
\text { (nCi) }\end{array}$ \\
\hline & 388D1 & 557.83 & 634 & 4.51 & 28.59 & 1.14 & 0.05 & & & 1.42 & & & & 1.34 & & & \\
\hline & 388D3 & 557.83 & 2252 & 2.4 & 54.05 & 4.04 & 0.10 & & & $\mathrm{~N} / \mathrm{A}$ & & & & $\mathrm{N} / \mathrm{A}$ & & & \\
\hline & & & & & & & & & & & & & & & & & \\
\hline \multirow[t]{9}{*}{\begin{tabular}{|l|l|} 
FD000583 \\
\end{tabular}} & T1583D1 & 277.12 & 2409 & 2.14 & 51.55 & 8.69 & 0.19 & 1.93 & 1.39 & & & & & 1.28 & & & \\
\hline & & & & & & & & & & & & & & & & & \\
\hline & T2583D1 & 300 & 404 & 5.68 & 22.95 & 1.35 & 0.08 & & & & & & & & & & \\
\hline & T2583D2 & 300 & 222 & 7.81 & 17.34 & 0.74 & 0.06 & 27.93 & 5.29 & & & & & 2.82 & & & \\
\hline & T2583D3 & 300 & 292 & 6.63 & 19.36 & 0.97 & 0.06 & & & & & & & & & & \\
\hline & & & & & & & & & & & & & & & & & \\
\hline & T3583D1 & 300.88 & 95 & 12.95 & 12.30 & 0.32 & 0.04 & & & & & & & & & & \\
\hline & 583D2 & 1021.6 & 146 & 11.43 & 16.69 & 0.14 & 0.02 & & & 0.76 & 1.21 & \begin{tabular}{|l|}
2582.79 \\
\end{tabular} & 69.74 & 0.40 & 0.71 & \begin{tabular}{|l|}
1516.05 \\
\end{tabular} & 40.93 \\
\hline & 583D3 & 1021.6 & 104 & 14.3 & 14.87 & 0.10 & 0.01 & & & 0.39 & & & & 0.24 & & & \\
\hline & & & & & & & & & & & & & & & & & \\
\hline \multirow[t]{9}{*}{\begin{tabular}{|l} 
FD000596 \\
\end{tabular}} & T1596D1 & 300 & 2248 & 2.23 & 50.13 & 7.49 & 0.17 & 2.63 & 1.62 & & & & & 1.43 & & & \\
\hline & T1596D2 & 300 & 906 & 3.71 & 33.61 & 3.02 & 0.11 & & & & & & & & & & \\
\hline & T1596D3 & 300 & 409 & 5.82 & 23.80 & 1.36 & 0.08 & & & & & & & & & & \\
\hline & & & & & & & & & & & & & & & & & \\
\hline & T2596D1 & 300 & 913 & 3.63 & 33.14 & 3.04 & 0.11 & & & & & & & & & & \\
\hline & T2596D2 & 300 & 847 & 3.86 & 32.69 & 2.82 & 0.11 & 8.35 & 2.89 & & & & & 2.05 & & & \\
\hline & T2596D3 & 300 & 540 & 5.01 & 27.05 & 1.80 & 0.09 & & & & & & & & & & \\
\hline & & & & & & & & & & & & & & & & & \\
\hline & & & & & & & & & & & & & & & & & \\
\hline
\end{tabular}


WSRC-TR-2001-00004

Revision 0

January 8, 2001

Page 65 of 69

\begin{tabular}{|c|c|c|c|c|c|c|c|c|c|c|c|c|c|c|c|c|c|}
\hline Sample & $\begin{array}{c}\text { File } \\
\text { Name }\end{array}$ & $\begin{array}{l}\text { Count } \\
\text { Time }\end{array}$ & Area & $\% \sigma$ & $\sigma$ & cps & $\sigma$ cps & $1 / T$ & \begin{tabular}{|c|}
$\mathbf{C f}=$ \\
SQRT( \\
1/T)
\end{tabular} & $\begin{array}{c}\text { Corr. } \\
\text { cps }\end{array}$ & $\begin{array}{l}\text { Corr. } \\
\text { Sum } \\
\text { (cps) }\end{array}$ & \begin{tabular}{|c|} 
Drum \\
Activity \\
(dps)
\end{tabular} & $\begin{array}{c}\text { Drum } \\
\text { Activity } \\
\text { (nCi) }\end{array}$ & $\begin{array}{l}C_{f} \text { of } \\
\ln 1 / T\end{array}$ & $\begin{array}{l}\text { Corr. } \\
\text { Sum } \\
(\text { cps })\end{array}$ & \begin{tabular}{|c|} 
Drum \\
Activity \\
(dps)
\end{tabular} & $\begin{array}{c}\text { Drum } \\
\text { Activity } \\
\text { (nCi) }\end{array}$ \\
\hline & T3596D1 & 300 & 585 & 4.74 & 27.73 & 1.95 & 0.09 & & & & & & & & & & \\
\hline & T3596D2 & 300 & 569 & 4.9 & 27.88 & 1.90 & 0.09 & & & & & & & & & & \\
\hline & T3596D3 & 300 & 1261 & 3.09 & 38.96 & 4.20 & 0.13 & 4.20 & 2.05 & & & & & \begin{tabular}{l|l|}
1.67 \\
\end{tabular} & & & \\
\hline & & & & & & & & & & & & & & & & & \\
\hline & 596D1 & 3600 & 4117 & 1.7 & 69.99 & 1.14 & 0.02 & & & 1.85 & & & & 1.63 & & & \\
\hline & 596D2 & 3600 & 2971 & 2.08 & 61.80 & 0.83 & 0.02 & & & 2.38 & 4.71 & 10053 & 271.43 & 1.70 & 3.71 & \begin{tabular}{|l|}
7919.99 \\
\end{tabular} & 213.84 \\
\hline & 596D3 & 3600 & 838 & 4.55 & 38.13 & 0.23 & 0.01 & & & 0.48 & & & & 0.39 & & & \\
\hline & & & & & & & & & & & & & & & & & \\
\hline FD000581 & T1581D1 & 105.61 & 1347 & 2.83 & 38.12 & 12.75 & 0.36 & 1.31 & 1.15 & & & & & \begin{tabular}{l|}
1.11 \\
\end{tabular} & & & \\
\hline & T1581D2 & 105.61 & 1211 & 3.04 & 36.81 & 11.47 & 0.35 & & & & & & & & & & \\
\hline & T1581D3 & 105.61 & 320 & 6.12 & 19.58 & 3.03 & 0.19 & & & & & & & & & & \\
\hline & & & & & & & & & & & & & & & & & \\
\hline & T2581D1 & 183.18 & 1972 & 2.33 & 45.95 & 10.77 & 0.25 & & & & & & & & & & \\
\hline & T2581D2 & 183.18 & 892 & 3.55 & 31.67 & 4.87 & 0.17 & 3.51 & 1.87 & & & & & 1.57 & & & \\
\hline & T2581D3 & 183.18 & 44 & 19.47 & 8.57 & 0.24 & 0.05 & & & & & & & & & & \\
\hline & & & & & & & & & & & & & & & & & \\
\hline & T3581D1 & 300 & 1453 & 2.76 & 40.10 & 4.84 & 0.13 & & & & & & & & & & \\
\hline & T3581D2 & 300 & 85 & 14.67 & 12.47 & 0.28 & 0.04 & & & & & & & & & & \\
\hline & T3581D3 & 300 & 295 & 6.74 & 19.88 & 0.98 & 0.07 & 19.37 & 4.40 & & & & & 2.58 & & & \\
\hline & & & & & & & & & & & & & & & & & \\
\hline & 581D1 & 3600 & 186 & 12.34 & 22.95 & 0.05 & 0.01 & & & 0.06 & & & & 0.06 & & & \\
\hline & 581D2 & 3600 & 442 & 6.73 & 29.75 & 0.12 & 0.01 & & & 0.23 & 0.83 & \begin{tabular}{|l|}
1766.60 \\
\end{tabular} & 47.70 & 0.19 & 0.57 & \begin{tabular}{|l|}
1207.61 \\
\end{tabular} & 32.61 \\
\hline & 581D3 & 3600 & 441 & 6.65 & 29.33 & 0.12 & \begin{tabular}{|l|}
0.01 \\
\end{tabular} & & & 0.54 & & & & \begin{tabular}{l|l}
0.32 \\
\end{tabular} & & & \\
\hline & & & & & & & & & & & & & & & & & \\
\hline 10/12 Acqu & disitions & & & & & & & & & & & & & & & & \\
\hline & & & & & & & & & & & & & & & & & \\
\hline & & & & & & & & & & & & & & & & & \\
\hline
\end{tabular}


WSRC-TR-2001-00004

Revision 0

January 8, 2001

Page 66 of 69

\begin{tabular}{|c|c|c|c|c|c|c|c|c|c|c|c|c|c|c|c|c|c|}
\hline Sample & $\begin{array}{c}\text { File } \\
\text { Name }\end{array}$ & $\begin{array}{l}\text { Count } \\
\text { Time }\end{array}$ & Area & $\% \sigma$ & $\sigma$ & cps & $\sigma$ cps & $1 / T$ & \begin{tabular}{|c|}
$\mathbf{C f}=$ \\
SQRT( \\
1/T)
\end{tabular} & $\begin{array}{c}\text { Corr. } \\
\text { cps }\end{array}$ & $\begin{array}{l}\text { Corr. } \\
\text { Sum } \\
\text { (cps) }\end{array}$ & \begin{tabular}{|c|} 
Drum \\
Activity \\
(dps)
\end{tabular} & $\begin{array}{c}\text { Drum } \\
\text { Activity } \\
\text { (nCi) }\end{array}$ & $\begin{array}{l}C_{f} \text { of } \\
\ln 1 / T\end{array}$ & $\begin{array}{l}\text { Corr. } \\
\text { Sum } \\
\text { (cps) }\end{array}$ & \begin{tabular}{|c|} 
Drum \\
Activity \\
(dps)
\end{tabular} & $\begin{array}{c}\text { Drum } \\
\text { Activity } \\
\text { (nCi) }\end{array}$ \\
\hline \multirow[t]{2}{*}{ T01 } & T01det1 & 60 & 902 & 3.47 & 31.30 & 15.03 & \begin{tabular}{|l|}
0.52 \\
\end{tabular} & & & & & & & & & & \\
\hline & & & & & & & & & & & & & & & & & \\
\hline \multirow[t]{2}{*}{ T02 } & T02det1 & 60 & 1036 & 3.17 & 32.84 & 17.27 & \begin{tabular}{|l|}
0.55 \\
\end{tabular} & & & & & & & & & & \\
\hline & & & & & & & & & & & & & & & & & \\
\hline \multirow[t]{2}{*}{ T03 } & T03det1 & 60 & 884 & 3.5 & 30.94 & 14.73 & \begin{tabular}{|l|}
0.52 \\
\end{tabular} & & & & & & & & & & \\
\hline & 0.00047 & 0.027 & & & & & & 15.68 & & & & & & & & & \\
\hline & & & & & & & & & & & & & & & & & \\
\hline \multirow[t]{15}{*}{\begin{tabular}{|l|} 
FD000597 \\
\end{tabular}} & T1597D1 & 156.85 & 1287 & 2.95 & 37.97 & 8.21 & \begin{tabular}{|l|}
0.24 \\
\end{tabular} & 1.94 & 1.39 & & & & & \begin{tabular}{l|l}
1.28 \\
\end{tabular} & & & \\
\hline & T1597D2 & 156.85 & 507 & 4.9 & 24.84 & 3.23 & \begin{tabular}{|l|}
0.16 \\
\end{tabular} & & & & & & & & & & \\
\hline & T1597D3 & 156.85 & 393 & 5.57 & 21.89 & 2.51 & \begin{tabular}{|l|}
0.14 \\
\end{tabular} & & & & & & & & & & \\
\hline & & & & & & & & & & & & & & & & & \\
\hline & T2597D1 & 314.41 & 789 & 3.91 & 30.85 & 2.51 & \begin{tabular}{|l|}
0.10 \\
\end{tabular} & & & & & & & & & & \\
\hline & T2597D2 & 314.41 & 1029 & 3.4 & 34.99 & 3.27 & \begin{tabular}{|l|}
0.11 \\
\end{tabular} & 5.75 & 2.40 & & & & & \begin{tabular}{l|}
1.84 \\
\end{tabular} & & & \\
\hline & T2597D3 & 314.41 & 368 & 4.07 & 14.98 & 1.17 & \begin{tabular}{|l|}
0.05 \\
\end{tabular} & & & & & & & & & & \\
\hline & & & & & & & & & & & & & & & & & \\
\hline & T3597D1 & 129.27 & 213 & 7.94 & 16.91 & 1.65 & \begin{tabular}{|l|}
0.13 \\
\end{tabular} & & & & & & & & & & \\
\hline & T3597D2 & 129.27 & 403 & 5.57 & 22.45 & 3.12 & \begin{tabular}{|l|}
0.17 \\
\end{tabular} & & & & & & & & & & \\
\hline & T3597D3 & 129.27 & 1285 & 2.96 & 38.04 & 9.94 & \begin{tabular}{|l|}
0.29 \\
\end{tabular} & \begin{tabular}{l|}
1.71 \\
\end{tabular} & \begin{tabular}{l|}
1.31 \\
\end{tabular} & & & & & \begin{tabular}{l|}
1.23 \\
\end{tabular} & & & \\
\hline & & & & & & & & & & & & & & & & & \\
\hline & 597D1 & 1800 & 211 & 10.16 & 21.44 & 0.12 & \begin{tabular}{|l|}
0.01 \\
\end{tabular} & & & 0.16 & & & & 0.15 & & & \\
\hline & 597D2 & 1800 & 979 & 3.69 & 36.13 & 0.54 & \begin{tabular}{|l|}
0.02 \\
\end{tabular} & & & 1.30 & 2.48 & 5283.95 & 142.67 & 1.00 & 2.10 & \begin{tabular}{|l|}
4473.02 \\
\end{tabular} & 120.77 \\
\hline & 597D3 & 1800 & 1392 & 3.06 & 42.60 & 0.77 & \begin{tabular}{|l|}
0.02 \\
\end{tabular} & & & 1.01 & & & & 0.95 & & & \\
\hline & & & & & & & & & & & & & & & & & \\
\hline \multirow[t]{5}{*}{ FD000595 } & T1595D1 & 151.16 & 1793 & 2.46 & 44.11 & 11.86 & \begin{tabular}{|l|}
0.29 \\
\end{tabular} & \begin{tabular}{l|}
1.32 \\
\end{tabular} & \begin{tabular}{l|}
1.15 \\
\end{tabular} & & & & & \begin{tabular}{l|l}
1.11 \\
\end{tabular} & & & \\
\hline & T1595D2 & 151.16 & 562 & 4.55 & 25.57 & 3.72 & \begin{tabular}{|l|}
0.17 \\
\end{tabular} & & & & & & & & & & \\
\hline & T1595D3 & 151.16 & 639 & 4.28 & 27.35 & 4.23 & \begin{tabular}{|l|}
0.18 \\
\end{tabular} & & & & & & & & & & \\
\hline & & & & & & & & & & & & & & & & & \\
\hline & & & & & & & & & & & & & & & & & \\
\hline
\end{tabular}


WSRC-TR-2001-00004

Revision 0

January 8, 200

Page 67 of 69

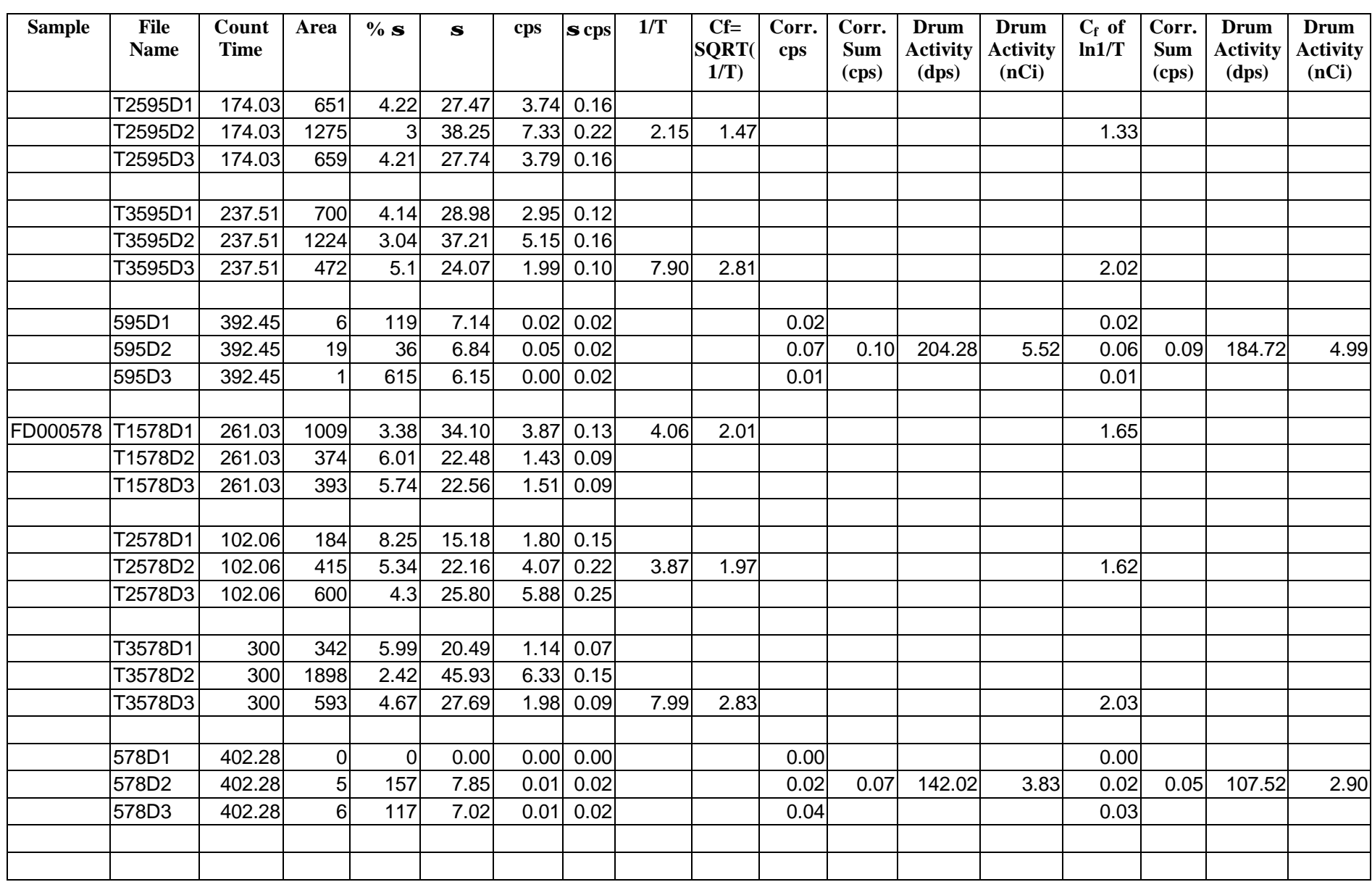


WSRC-TR-2001-00004

Revision 0

January 8, 2001

Page 68 of 69

\begin{tabular}{|c|c|c|c|c|c|c|c|c|c|c|c|c|c|c|c|c|c|}
\hline Sample & $\begin{array}{c}\text { File } \\
\text { Name }\end{array}$ & $\begin{array}{l}\text { Count } \\
\text { Time }\end{array}$ & Area & $\% \sigma$ & $\sigma$ & cps & $\sigma$ cps & $1 / \mathrm{T}$ & \begin{tabular}{|c|}
$\mathbf{C f}=$ \\
SQRT ( \\
$\mathbf{1 / T})$
\end{tabular} & $\begin{array}{c}\text { Corr. } \\
\text { cps }\end{array}$ & $\begin{array}{l}\text { Corr. } \\
\text { Sum } \\
\text { (cps) }\end{array}$ & \begin{tabular}{|c|} 
Drum \\
Activity \\
(dps)
\end{tabular} & \begin{tabular}{|c|} 
Drum \\
Activity \\
(nCi)
\end{tabular} & $\begin{array}{l}C_{f} \text { of } \\
\ln 1 / T\end{array}$ & \begin{tabular}{|l|} 
Corr. \\
Sum \\
(cps)
\end{tabular} & \begin{tabular}{|c|} 
Drum \\
Activity \\
(dps)
\end{tabular} & $\begin{array}{c}\text { Drum } \\
\text { Activity } \\
\text { (nCi) }\end{array}$ \\
\hline \multirow[t]{10}{*}{\begin{tabular}{|l|} 
FD000414 \\
\end{tabular}} & T1414D1 & 154.69 & 1971 & 2.38 & 46.91 & 12.74 & 0.30 & 2.94 & 1.72 & & & & & 1.48 & & & \\
\hline & T1414D3 & 154.69 & 468 & 5.13 & 24.01 & 3.03 & 0.16 & & & & & & & & & & \\
\hline & & & & & & & & & & & & & & & & & \\
\hline & T2414D1 & 63.11 & 771 & 3.84 & 29.61 & 12.22 & \begin{tabular}{|l|}
0.47 \\
\end{tabular} & & & & & & & & & & \\
\hline & & & & & & & & & & & & & & & & & \\
\hline & T3414D1 & 300 & 2385 & 2.19 & 52.23 & 7.95 & \begin{tabular}{|l|}
0.17 \\
\end{tabular} & & & & & & & & & & \\
\hline & T3414D2 & 300 & 2408 & 2.22 & 53.46 & 8.03 & 0.18 & & & & & & & & & & \\
\hline & T3414D3 & 300 & 981 & 3.61 & 35.41 & 3.27 & \begin{tabular}{|l|}
0.12 \\
\end{tabular} & 26.67 & 5.16 & & & & & 2.79 & & & \\
\hline & & & & & & & & & & & & & & & & & \\
\hline & 414D1 & 655.81 & 4864 & 1.53 & \begin{tabular}{l|}
74.42 \\
\end{tabular} & 7.42 & \begin{tabular}{|l|}
0.11 \\
\end{tabular} & & & 12.73 & & & & 11.00 & & & \\
\hline \multirow{12}{*}{\begin{tabular}{|l} 
FD000402 \\
\end{tabular}} & T1402D2 & 324.1 & 1674 & 2.62 & 43.86 & 5.17 & \begin{tabular}{|l|}
0.14 \\
\end{tabular} & & & & & & & & & & \\
\hline & T1402D3 & 324.1 & 408 & 5.87 & 23.95 & 1.26 & \begin{tabular}{|l|}
0.07 \\
\end{tabular} & & & & & & & & & & \\
\hline & & & & & & & & & & & & & & & & & \\
\hline & T2402D1 & 300 & 1445 & 2.81 & 40.60 & 4.82 & \begin{tabular}{|l|}
0.14 \\
\end{tabular} & & & & & & & & & & \\
\hline & T2402D2 & 300 & 678 & 4.27 & 28.95 & 2.26 & 0.10 & 9.35 & 3.06 & & & & & 2.12 & & & \\
\hline & T2402D3 & 300 & 773 & 4.9 & 37.88 & 2.58 & \begin{tabular}{|l|}
0.13 \\
\end{tabular} & & & & & & & & & & \\
\hline & & & & & & & & & & & & & & & & & \\
\hline & T3402D1 & 300 & 569 & 4.62 & 26.29 & 1.90 & \begin{tabular}{|l|}
0.09 \\
\end{tabular} & & & & & & & & & & \\
\hline & T3402D2 & 300 & 673 & 4.23 & 28.47 & 2.24 & \begin{tabular}{|l|}
0.09 \\
\end{tabular} & & & & & & & & & & \\
\hline & T3402D3 & 300 & 1072 & 3.28 & 35.16 & 3.57 & \begin{tabular}{|l|}
0.12 \\
\end{tabular} & 5.35 & 2.31 & & & & & 1.80 & & & \\
\hline & & & & & & & & & & & & & & & & & \\
\hline & 402D1 & 1200 & 392 & 6.12 & 23.99 & 0.33 & \begin{tabular}{|l|}
0.02 \\
\end{tabular} & & & 0.67 & & & & 0.55 & & & \\
\hline
\end{tabular}


WSRC-TR-2001-00004

Revision 0

January 8, 2001

Page 69 of 69

\begin{tabular}{|c|c|c|c|c|c|c|c|c|c|c|c|c|c|c|c|c|c|}
\hline Sample & $\begin{array}{c}\text { File } \\
\text { Name }\end{array}$ & $\begin{array}{l}\text { Count } \\
\text { Time }\end{array}$ & Area & $\% \sigma$ & $\sigma$ & cps & $\sigma$ cps & $1 / T$ & $\begin{array}{c}\text { Cf }= \\
\text { SQRT( } \\
\text { 1/T) }\end{array}$ & $\begin{array}{c}\text { Corr. } \\
\text { cps }\end{array}$ & $\begin{array}{l}\text { Corr. } \\
\text { Sum } \\
\text { (cps) }\end{array}$ & \begin{tabular}{|c|} 
Drum \\
Activity \\
(dps)
\end{tabular} & \begin{tabular}{|c|} 
Drum \\
Activity \\
(nCi)
\end{tabular} & $\begin{array}{l}C_{f} \text { of } \\
\ln 1 / T\end{array}$ & $\begin{array}{l}\text { Corr. } \\
\text { Sum } \\
\text { (cps) }\end{array}$ & \begin{tabular}{|c|} 
Drum \\
Activity \\
(dps)
\end{tabular} & \begin{tabular}{|c|} 
Drum \\
Activity \\
(nCi)
\end{tabular} \\
\hline & 402D2 & 1200 & 699 & 4.33 & 30.27 & 0.58 & 0.03 & & & 1.78 & 3.94 & 8397.97 & 226.75 & 1.24 & 2.94 & 6262.51 & 169.09 \\
\hline
\end{tabular}

